\title{
The parabolic Anderson model on Riemann surfaces
}

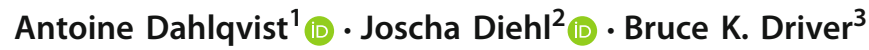 \\ Received: 23 February 2017 / Revised: 2 March 2018 / Published online: 23 July 2018 \\ (c) The Author(s) 2018
}

\begin{abstract}
We show well-posedness for the parabolic Anderson model on 2-dimensional closed Riemannian manifolds. To this end we extend the notion of regularity structures to curved space, and explicitly construct the minimal structure required for this equation. A central ingredient is the appropriate re-interpretation of the polynomial model, which we build up to any order.
\end{abstract}

Mathematics Subject Classification 60H15 · 35R01

\section{Contents}

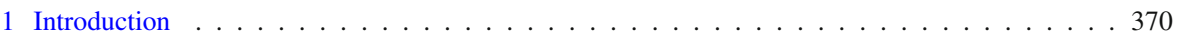

1.1 Notation . . . . . . . . . . . . . . . . . . . . . . . . . . . . 372

2 Hölder spaces . . . . . . . . . . . . . . . . . . . . . . . . . . . 372

3 Regularity structures on manifolds . . . . . . . . . . . . . . . . . . . . . . . . . 377

4 Linear "polynomials" on a Riemannian manifold . . . . . . . . . . . . . . . . . . . . . . . . 386

5 The regularity structure for PAM on a manifold . . . . . . . . . . . . . . . . . . . . . . 389

6 Schauder estimates . . . . . . . . . . . . . . . . . . . . . . . . . . 392

7 Fixpoint argument . . . . . . . . . . . . . . . . . . . . . . . . . 408

8 The Gaussian model . . . . . . . . . . . . . . . . . . . . . . . . . . . . . 410

9 Appendix - Higher order "polynomials" . . . . . . . . . . . . . . . . . . . . . 417

9.1 Higher order covariant derivatives . . . . . . . . . . . . . . . . . . . . . . 418

9.1.1 Symmetric parts of covariant derivatives determine all derivatives . . . . . . . . . . . 4424

9.2 The polynomial regularity structure and model . . . . . . . . . . . . . . . . . . . . . . . 428

B. Driver: The author's main contribution was to the Appendix of this paper.

Joscha Diehl

joscha.diehl@gmail.com

Antoine Dahlqvist

antoine.dahlqvist@gmail.com

1 University College Dublin, Belfield Campus, Dublin 4, Dublin, Ireland

2 Max-Planck Institute for Mathematics in the Sciences, Inselstraße 22, Leipzig 04103, Germany

3 Department of Mathematics, University of California, San Diego 9500 Gilman Drive La Jolla, California 92093-0112, USA 
9.3 A reformulation: the jet bundle . . . . . . . . . . . . . . . . . . . . 438

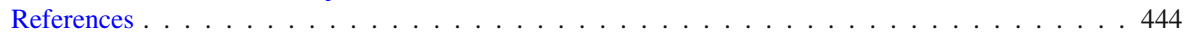

\section{Introduction}

The last few years have seen an explosion of literature on singular stochastic partial differential equations (singular SPDEs). The simplest instance of such an equation is the parabolic Anderson model in two dimensions, formally written as

$$
\partial_{t} u=\Delta u+u \xi
$$

Here $u:[0, T] \times D \rightarrow \mathbb{R}$ is looked for, where $D$ is some 2 dimensional domain, and $\xi$ is (time-independent) white noise on the domain $D$. This equation is formally ill-posed (or "singular"), since $u$ is not expected to be regular enough for the product $u \xi$ to be well-defined analytically. The standard tool of stochastic calculus, the Itō integral, is also of no use here, since the white-noise is constant in time.

With the breakthrough results of Hairer [9] and Gubinelli, Imkeller and Perkowski [7] a large class of such equations has become amenable to analysis. Let us sketch the approach of [9], since this is the one we shall use in this work.

- assume that $u$ "looks like" the solution $v$ to the additive-noise equation

$$
\partial_{t} v=\Delta v+\xi
$$

which is classically well-defined via convolution with the heat semigroup $P_{t}$

- under this assumption, if we somehow define $v \cdot \xi$, then the framework defines $u \cdot \xi$ automatically

- close the fixpoint argument, i.e.

1. $u$ "looks like" $v$

2. $w:=P_{t} u_{0}+\int_{0}^{t} P_{t-s}\left[u_{s} \xi\right] d s$

3. then $w$ "looks like" $v$

It then only remains to define the missing ingredient " $v \cdot \xi$ ". This can be done probabilistically and is actually the only place in this theory that is not deterministic. Using this procedure, it is shown in [9] that (PAM) possesses a unique solution for $D=\mathbb{T}^{2}$, the two dimensional torus.

In this work we show that the theory can be adapted to work for $D=M$, a 2-dimensional closed Riemannian manifold. The theory of regularity structures is intrinsically a local theory (as opposed to the theory of paracontrolled distributions, which, at least at first sight is global in spirit). It is hence natural to expect that it can be applied to general geometries. It turns out that the implementation of this heuristic is not straightforward.

At least two hurdles need indeed to be bypassed. On the one hand, at the core of Euclidean regularity structures stands the space of polynomials, encoding classical Taylor expansions at any point. The operation of re-expansion from a point to another leads to a morphism from $\left(\mathbb{R}^{d},+\right)$ to a space of unipotent matrices. On a manifold, 
one would need to look for such a space, encoding Taylor expansion and enjoying a similar structure. On the other hand, as usual for fixpoint arguments of (S)PDEs, one needs to estimate the improvement of the heat kernel in adequate spaces, which is a global operation (Schauder estimates).

To solve the first issue, we show that the space of polynomials on the tangent space of the manifolds is a suitable candidate for a canonical regularity structure, that allows to encode Hölder functions. This choice enforces a modified definition of a regularity structure. In particular one has to abandon the idea of one fixed vector space and work with vector bundles instead. For our definition of a model, there is no unipotent structure anymore and re-expansions are only approximately compatible. Within this new framework, when considering the parabolic Anderson model on a surface, we give a weak version of a Schauder estimate with elementary tools and heat kernel estimates.

This exposition does not demand any previous knowledge of regularity structures on the reader. In this sense it is self-contained, apart from a reference to the reconstruction theorem of Hairer in our Theorem 21 and in the construction of the Gaussian model in Sect. 8. Its proof using wavelet analysis is of no use reproducing here. We believe that the validity of that reconstruction theorem, which we use in coordinates, is easily believed.

We follow a very hands-on approach. Instead of trying to set up a general theory of regularity structures on manifolds, we work with the smallest structure that is necessary to solve PAM. We show the Schauder estimates explicitly. Apart from introducing for the first time regularity structures on manifolds, we believe our work also has a pedagogical value. Since everything is laid out explicitly and covers the flat case $M=\mathbb{T}^{2}$, it can serve as a gentle introduction to the general theory.

In future work we will investigate the algebraic foundation necessary for studying general equations, without having to build the regularity structure "by hand". For general equations a new proof of the Schauder estimates has also to be found.

During the writing of the present article, a different approached has been put forward in [2], where the notion of paracontrolled products using semi-groups is developed on general metric spaces. The advantage of the paracontrolled approach is that it requires less machinery. On the downside, the class of equations that can be covered is currently strictly smaller than in the setting of regularity structures. Let us point to [3] though, which pushes the framework to more general equations.

The outline of this paper is as follows. After presenting notational conventions, we give in Sect. 2 the notion of distributions on manifold we shall use in this work. Moreover we introduce Hölder spaces on manifolds. In Sect. 3 we introduce the notion of regularity structure, model and modelled distribution on a manifold. We show how these objects behave nicely under diffeomorphisms and use this fact to show the reconstruction theorem. In Sect. 4 we give the simplest non-trivial example of a regularity structure on a manifold; the regularity structure for "linear polynomials". This forms the basis for the regularity structure for PAM, which is constructed in Sect. 5. As input it takes the product $\nu \xi$ alluded to before. This is constructed in Sect. 8 via renormalization. Section 6 gives the Schauder estimate for modelled distributions in the setting of PAM and finally Sect. 7 solves the corresponding fixpoint equation. In Sect. 9 we show how the construction of Sect. 4 can be extended to "polynomials" of arbitrary order. 


\subsection{Notation}

In all what follows $M$ will be a $d$-dimensional closed Riemannian manifold. When we specialize to the parabolic Anderson model (PAM), the dimension will be $d=2$. Denote by $\delta>0$ the radius of injectivity of $M$. For $p \in M$ we denote with $\exp _{p}$ : $T_{p} M \rightarrow M$ the exponential function. It is a diffeomorphism on $B_{T_{p} M}\left(0_{p}, \delta\right):=\{x \in$ $\left.T_{p} M:|x|_{T_{p} M}<\delta\right\}$, with inverse $\exp _{p}^{-1}$.

For a function $\varphi$ supported in $B_{T_{p} M}\left(0_{p}, \delta\right)$ define for $\lambda \in(0,1], p \in M$,

$$
\varphi_{p}^{\lambda}(\cdot):=\lambda^{-d} \varphi\left(\lambda^{-1} \exp _{p}^{-1}(\cdot)\right)
$$

extended to all of $M$ by setting it to zero outside of $B_{T_{p} M}\left(0_{p}, \delta\right)$.

For $\tau \in \mathcal{G}, \mathcal{G}$ a graded normed vector bundle with grading $A$ we denote by $\|\tau\|_{a}$ the size of component in the $a$-th level, $a \in A$.

The differential of a smooth enough function $f: M \rightarrow \mathbb{R}$ at a point $p$ will be denoted $\left.d\right|_{p} f \in T_{p}^{*} M$. Similarly for higher order derivatives (see Sect. 9) $\left.\nabla^{\ell}\right|_{p} f \in$ $\left(T_{p}^{*} M\right)^{\otimes \ell}$. For the action on vectors $W \in\left(T_{p} M\right)^{\otimes \ell}$, we shall write either $\left\langle\left.\nabla^{\ell}\right|_{p} f, W\right\rangle$ or $\nabla_{W} f$.

For $p \in M, r, \delta>0$ denote

$$
\mathcal{B}_{T_{p} M}^{r, \delta}:=\left\{\varphi \in C^{r}\left(T_{p} M\right): \operatorname{supp} \varphi \subset B_{T_{p} M}\left(0_{p}, \delta\right),\|\varphi\|_{C^{r}\left(\mathbb{R}^{d}\right)} \leq 1\right\},
$$

Here $r$ will depend on the situation, and will always be large enough so that the distributions under consideration can act on $\varphi$.

We shall use $p, q$ for points in $M$ and $x, y, z$ to denote points in $\mathbb{R}^{d}$. For $x \in \mathbb{R}^{d}$, $\varphi: \mathbb{R}^{d} \rightarrow \mathbb{R}$ we write

$$
\varphi_{x}^{\lambda}:=\lambda^{-d} \varphi\left(\lambda^{-1}(\cdot-x)\right),
$$

which is consistent with the notation introduced above when considering $\mathbb{R}^{d}$ as Riemannian manifold with the standard metric. We also define, analogously to above, $B_{\mathbb{R}^{d}}(0, \delta)$ and $\mathcal{B}_{\mathbb{R}^{d}}^{r, \delta}$. Balls in $M$ are denote by $B_{M}(p, \delta):=\{q \in M: d(q, p)<\delta\}$.

For $\gamma \in \mathbb{R}$ we denote by $[\gamma]$ the largest integer strictly smaller than $\gamma$.

For a pairing of a distribution $T$ with a test function we write $\langle T, \varphi\rangle$.

For two quantities $f, g$ we write $f \lesssim g$ if there exists a constant $C>0$ such that $f \leq C g$. To make explicit the dependence of $C$ on a quantity $h$, we sometimes write $f \lesssim h g$.

We denote the positive natural numbers by $\mathbb{N}$ and the non-negative ones by $\mathbb{N}_{0}$.

\section{Hölder spaces}

Definition 1 A distribution on $M$ is a bounded, linear functional on $C_{c}^{\infty}(M)(=$ $C^{\infty}(M)$, if $M$ is compact). 
Given a density $\lambda$ on $M,\left\langle T_{\lambda}, \varphi\right\rangle:=\int_{M} \varphi d \lambda$ defines a distribution. Distributions are hence "generalized densities". Compare [6, Section 2.8] and [17, Section 1.3].

There is another definition of distributions as "generalized functions", see [12, Section 1.8]. They are equivalent when there is a canonical way to turn a function into a density and vice versa. This is the case when there is a reference density, like on a Riemannian manifold.

Remark 2 On a Riemannian manifold $M$, denote the standard density by $d|\mathrm{Vol}|$. We can lift a function $f \in C(M)$ to a density $f d|\mathrm{Vol}|$. Then, for $f \in C_{c}^{\infty}(M), T_{f}$ defined as

$$
\left\langle T_{f}, \varphi\right\rangle:=\int_{M} f(z) \varphi(z) d|\operatorname{Vol}|,
$$

is a distribution.

Definition 3 (Push-forward) Let $(\Psi, \mathcal{U})$ be a coordinate chart on $M$. If $\varphi \in C_{c}^{\infty}(\Psi(\mathcal{U})$ ) and $T$ is a distribution on $M$ we can define the push-forward $\Psi_{*} T \in \mathcal{D}^{\prime}(\Psi(\mathcal{U}))$ via

$$
\left\langle\Psi_{*} T, \varphi\right\rangle:=\left\langle T, \Psi^{*} \varphi\right\rangle:=\langle T, \varphi \circ \Psi\rangle \text {. }
$$

Remark 4 This push-forward is compatible with the pull-back of densities. Indeed, for $f \in C(M)$ we get the distribution $T_{f}:=f d \mid$ Vol $\mid$, by Remark 2 . This density pulls back under $\Psi^{-1}$ as (compare [13, Proposition 16.38])

$$
f d|\mathrm{Vol}| \mapsto f \circ \Psi^{-1} \sqrt{\operatorname{det} g} d y^{1} \wedge \cdots \wedge d y^{d},
$$

where $y^{i}$ are standard coordinates on $\mathbb{R}^{d}$, and $g$ is the Riemannian metric in the coordinates $\Psi$. Hence

$$
\begin{aligned}
\left\langle\Psi_{*} T_{f}, \varphi\right\rangle & =\left\langle T_{f}, \varphi \circ \Psi\right\rangle \\
& =\int_{M} \varphi(\Psi(x)) f(x) d|\mathrm{Vol}| \\
& =\int_{\mathbb{R}^{d}} \varphi(z) f\left(\Psi^{-1}(z)\right) \sqrt{\operatorname{det} g(z)} d z \\
& =\left\langle\bar{T}_{\left(\Psi^{-1}\right)^{*}(f d|\mathrm{Vol}|)}, \varphi\right\rangle,
\end{aligned}
$$

where the last line is the pairing of a distribution with a test function on $\mathbb{R}^{d}$ and $\bar{T}_{h}$ is the canonical identification of a locally integrable density $h$ on $\mathbb{R}^{d}$ with a distribution.

Recall the following definition of Hölder spaces in Euclidean space.

Definition 5 For $\gamma>0$ we keep the classical definition, that is

$$
\|T\|_{C^{\gamma}\left(\mathbb{R}^{d}\right)}:=\sum_{|\ell| \leq n}\left\|D^{\ell} T\right\|_{\infty ; \mathbb{R}^{d}}+\sup _{|\ell|=n ; x, y \in \mathbb{R}^{d}} \frac{\left|D^{\ell} T(x)-D^{\ell} T(y)\right|}{|x-y|^{s}},
$$

where $\gamma=n+s, n \in \mathbb{N}_{0}, s \in(0,1]$. 
For $\gamma \leq 0$ denote by $C^{\gamma}\left(\mathbb{R}^{d}\right)$ the space of distributions $T \in \mathcal{D}^{\prime}\left(\mathbb{R}^{d}\right)$ with

$$
\|T\|_{C \gamma\left(\mathbb{R}^{d}\right)}:=\sup _{x \in \mathbb{R}^{d}} \sup _{\lambda \in(0,1]} \sup _{\varphi \in \mathcal{B}_{\mathbb{R}^{r}}^{r, 1}} \lambda^{-\gamma}\left|\left\langle T, \varphi_{x}^{\lambda}\right\rangle\right|<\infty
$$

Here $r:=-[\gamma]$, and $\varphi_{x}^{\lambda}$ as well as the set of test functions $\mathcal{B}_{\mathbb{R}^{d}}^{r, 1}$ are defined in Sect. 1.1.

Remark 6 For $\gamma<0$ the norm is independent of the arbitrary upper bound 1 for the supremum over $\lambda$ as well as the support of $\varphi$ : for every $\lambda_{0}, \varepsilon_{0}>0$

$$
\|T\|_{C \gamma\left(\mathbb{R}^{d}\right)} \lesssim \lambda_{0}, \varepsilon_{0} \sup _{x \in \mathbb{R}^{d}} \sup _{\lambda \in\left(0, \lambda_{0}\right]} \sup _{\varphi \in \mathcal{B}_{\mathbb{R}^{r}}^{r, \varepsilon_{0}}} \lambda^{-\gamma}\left|\left\langle T, \varphi_{x}^{\lambda}\right\rangle\right|,
$$

where $r:=-[\gamma]$.

Remark 7 Every time that a condition like

$$
\left|\left\langle T, \varphi_{x}^{\lambda}\right\rangle\right| \lesssim \lambda^{\gamma}, \lambda \in(0,1]
$$

appears, for some $\varepsilon>0$, uniformly over supp $\varphi \subset B_{\mathbb{R}^{d}}(0, \varepsilon)$, with $\|\varphi\|_{C^{r}\left(B_{\mathbb{R}^{d}}(0, \varepsilon)\right) \leq} \leq$ 1 , one can equivalently demand for some $\varepsilon>0$

$$
|\langle T, \varphi\rangle| \lesssim \lambda^{\gamma}
$$

uniformly over $\lambda \in(0,1]$ and $\operatorname{supp} \varphi \subset B_{\mathbb{R}^{d}}(x, \lambda \varepsilon)$, with $\left\|D^{k} \varphi\right\|_{\infty} \lesssim \lambda^{-d-k}$, for $k=0, \ldots, r$.

We need a reformulation similar to this remark, but for Schwartz test functions.

Lemma 8 Let $\gamma \leq 0$ and $T \in C^{\gamma}\left(\mathbb{R}^{d}\right)$. Then $T \in S^{\prime}\left(\mathbb{R}^{d}\right)$ (and not just $T \in \mathcal{D}^{\prime}\left(\mathbb{R}^{d}\right)$ ). Moreover, define for $\varphi \in S\left(\mathbb{R}^{d}\right), \lambda \in(0,1], x_{0} \in \mathbb{R}^{d}, N \in \mathbb{N}$ and $r:=-[\gamma]$

$$
C\left(\varphi, \lambda, x_{0}, N, r\right):=\sup _{|k| \leq r} \sup _{x \in \mathbb{R}^{d}}\left|D^{k} \varphi(x)\right| \lambda^{d+k}\left(1+\lambda^{-N}\left|x-x_{0}\right|^{N}\right) .
$$

Then, for $N>d$,

$$
|\langle T, \varphi\rangle| \lesssim_{N} C\left(\varphi, \lambda, x_{0}, N, r\right)\|T\|_{C \gamma\left(\mathbb{R}^{d}\right)} \lambda^{\gamma}
$$

Remark 9 Note that if $\varphi \in S\left(\mathbb{R}^{d}\right)$, then $\varphi_{x_{0}}^{\lambda}:=\lambda^{-d} \varphi\left(\lambda^{-1}\left(\cdot-x_{0}\right)\right)$ satisfies for $\lambda \in$ $(0,1], N \in \mathbb{N}, x_{0} \in \mathbb{R}^{d}$ and $r>0$,

$$
C\left(\varphi_{x_{0}}^{\lambda}, \lambda, x_{0}, N, r\right) \leq \sup _{|k| \leq r} \sup _{x \in \mathbb{R}^{d}}\left|D^{k} \varphi(x)\right|\left(1+|x|^{N}\right)<\infty
$$


Proof Let $\phi_{z}, z \in \mathbb{Z}^{d}$, be a partition of unity of $\mathbb{R}^{d}$ such that $\operatorname{supp} \phi_{z} \subset B_{\mathbb{R}^{d}}(z, 1)$ and $\sup _{z \in \mathbb{Z}^{d}}\left\|\phi_{z}\right\|_{C^{r}}<\infty$. Define

$$
\varphi_{z, \lambda}(\cdot):=\phi_{z}\left(\lambda^{-1} \cdot\right) \varphi(\cdot)
$$

Then $\sum_{z \in \mathbb{Z}^{d}} \varphi_{z, \lambda}=\varphi$. Write for short $C_{\varphi}:=C\left(\varphi, \lambda, x_{0}, N, r\right)$. We have $\operatorname{supp} \varphi_{z, \lambda} \subset B_{\mathbb{R}^{d}}(\lambda z, \lambda)$ and, since $\varphi \in S\left(\mathbb{R}^{d}\right)$,

$$
\begin{aligned}
\left\|D^{k} \varphi_{z, \lambda}\right\|_{\infty} & \lesssim C_{\varphi} \lambda^{-d-k} \frac{1}{1+\lambda^{-N}\left|\lambda z-x_{0}\right|^{N}} \\
& =C_{\varphi} \lambda^{-d-k} \frac{1}{1+\left|z-\lambda^{-1} x_{0}\right|^{N}}
\end{aligned}
$$

Then

$$
\begin{aligned}
|\langle T, \varphi\rangle| & \leq \sum_{z \in \mathbb{Z}^{d}}\left|\left\langle T, \varphi_{z, \lambda}\right\rangle\right| \\
& \leq C_{\varphi}\|T\|_{C^{\gamma}\left(\mathbb{R}^{d}\right)} \lambda^{\gamma} \sum_{z \in \mathbb{Z}^{d}} \frac{1}{1+\left|z-\lambda^{-1} x_{0}\right|^{N}} \\
& \lesssim{ }_{N} C_{\varphi}\|T\|_{C \gamma\left(\mathbb{R}^{d}\right)} \lambda^{\gamma},
\end{aligned}
$$

as desired. We used the fact that $\sum_{z \in \mathbb{Z}^{d}} \frac{1}{1+\left|z-\lambda^{-1} x_{0}\right|^{N}}$ is upper bounded by $\int_{\mathbb{R}^{d}} \frac{1}{1+\left|z-\lambda^{-1} x_{0}\right|^{N}} d z=\int_{\mathbb{R}^{d}} \frac{1}{1+|z|^{N}} d z$, which is finite, since $N>d$, and independent of $\lambda$.

Definition 10 Let $M$ be a closed Riemannian manifold. Let a finite partition of unity $\left(\phi_{i}\right)_{i \in I}$ be given on $M$, subordinate to a finite atlas $\left(\Psi_{i}, U_{i}\right)_{i \in I}$. For $\gamma \in \mathbb{R}$, define

$C^{\gamma}(M):=C^{\gamma}\left(M ;\left(\Psi_{i}, U_{i}\right), \phi_{i}\right):=\left\{f: M \rightarrow \mathbb{R}:\left(\Psi_{i}\right)_{*}\left(\phi_{i} f\right) \in C^{\gamma}\left(\mathbb{R}^{d}\right), \quad \forall i \in I\right\}$,

and

$$
\|f\|_{\gamma}:=\sup _{i \in I}\left\|\left(\Psi_{i}\right)_{*}\left(\phi_{i} f\right)\right\|_{C^{\gamma}\left(\mathbb{R}^{d}\right)}
$$

For $\gamma>0$, an equivalent characterization of $C^{\gamma}(M)$ will be shown in Theorem 92. We now give one in the case $\gamma \leq 0$.

Lemma 11 For $\gamma \leq 0, M$ a closed Riemannian manifold, an equivalent norm on $C^{\gamma}(M)$ is given by

$$
\sup _{p \in M, \lambda \in(0,1], \varphi \in \mathcal{B}_{T_{p} M}^{r, \delta}} \frac{\left|\left\langle T, \varphi_{p}^{\lambda}\right\rangle\right|}{\lambda \gamma}
$$

where we recall that $\varphi_{p}^{\lambda}$ is defined in (2). 
Proof Fix a finite atlas $\left(\Psi_{i}, U_{i}\right)_{i \in I}$ with subordinate partition of unity $\phi_{i}$. Denote

$$
\begin{aligned}
C_{1} & :=\|T\|_{C \gamma}\left(M ;\left(\Psi_{i}, U_{i}\right), \phi_{i}\right) \\
C_{2} & :=\sup _{p \in M, \lambda \in(0,1], \varphi \in \mathcal{B}_{T_{p} M}^{r, \delta}} \frac{\left|\left\langle T, \varphi_{p}^{\lambda}\right\rangle\right|}{\lambda \gamma} .
\end{aligned}
$$

$\left(C_{2} \lesssim C_{1}\right):$ Let $\varphi \in \mathcal{B}_{T_{p} M}^{r, \delta}, p \in M$. Then

$$
\begin{aligned}
\left\langle T, \varphi_{p}^{\lambda}\right\rangle & =\sum_{i}\left\langle T \phi_{i}, \varphi_{p}^{\lambda}\right\rangle \\
& =\sum_{i}\left\langle\left(\Psi_{i}\right)_{*}\left(T \phi_{i}\right), \varphi_{p}^{\lambda} \circ \Psi_{i}^{-1}\right\rangle \\
& =\sum_{i}\left\langle\left(\Psi_{i}\right)_{*}\left(T \phi_{i}\right), \tilde{\phi}_{i} \circ \Psi_{i}^{-1} \varphi_{p}^{\lambda} \circ \Psi_{i}^{-1}\right\rangle \\
& =\sum_{i}\left\langle\left(\Psi_{i}\right)_{*}\left(T \phi_{i}\right), \eta_{i}\right\rangle
\end{aligned}
$$

with $\eta_{i}:=\left(\tilde{\phi}_{i} \varphi_{p}^{\lambda}\right) \circ \Psi_{i}^{-1}$. Here the functions $\tilde{\phi}_{i}$ are chosen such that supp $\tilde{\phi}_{i} \subset U_{i}$ and $\phi_{i} \tilde{\phi}_{i}=\phi_{i}$. Now, $\varphi_{p}^{\lambda}$ is supported in a ball of radius $\delta \lambda$ around $p$, hence for some $c>0$ independent of $i$

$$
\operatorname{supp} \eta_{i} \subset \Psi_{i}\left(B_{M}(p, \delta \lambda) \cap \operatorname{supp} \tilde{\phi}_{i}\right) \subset B_{\mathbb{R}^{d}}\left(z_{i}, c \lambda\right)
$$

for some $z_{i} \in \mathbb{R}^{d}$. Here, the last inclusion follows from the fact that the atlas is finite and $\tilde{\phi}_{i}$ is strictly contained in $U_{i}$, so that the Lipschitz norm of $\Psi_{i}$ and $\Psi_{i}^{-1}$ are uniformly bounded in the regions of interest. By the same reasoning, the derivatives up to order $r$ of $\exp _{p}^{-1} \circ \Psi_{i}^{-1}$ are uniformly bounded on the relevant regions. Hence

$$
\left\|D^{k} \eta_{i}\right\|_{\infty} \lesssim C_{1} \lambda^{-d-k}
$$

and the result follows from Remark 7.

$\left(C_{1} \lesssim C_{2}\right)$ : By Remark 6, we have to show for some $\lambda_{0}>0$ and for all $i \in I$, $\operatorname{supp} \varphi \subset B_{\mathbb{R}^{d}}(0,1),\|\varphi\|_{C^{r}} \leq 1$ and $x \in \mathbb{R}^{d}$

$$
\left|\left\langle\left(\Psi_{i}\right)_{*}\left(T \phi_{i}\right), \varphi_{x}^{\lambda}\right\rangle\right| \lesssim C_{2} \lambda^{\gamma}, \quad \lambda \in\left(0, \lambda_{0}\right]
$$

Since $\phi_{i}$ is supported away from the boundary of $U_{i}$, there exists $\lambda_{0}>0$ such that for all $x \in \mathbb{R}^{d}$ there is some $p \in M$ such that $\operatorname{supp} \varphi_{i ; \lambda_{0} ; x} \subset B_{M}(p, \delta)$. ${ }^{1}$ Since the atlas is finite, $\lambda_{0}$ can be chosen uniformly for all $i \in I$.

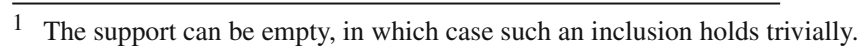


Then, for $\lambda \leq \lambda_{0}$

$$
\begin{aligned}
\left\langle\left(\Psi_{i}\right)_{*}\left(T \phi_{i}\right), \varphi_{x}^{\lambda}\right\rangle & =\left\langle T, \varphi_{x}^{\lambda} \circ \Psi_{i} \phi_{i}\right\rangle \\
& =\left\langle T,\left(\varphi_{x}^{\lambda} \circ \Psi_{i} \phi_{i}\right) \circ \exp _{p} \circ \exp _{p}^{-1}\right\rangle,
\end{aligned}
$$

Now, one checks that $\varphi_{i ; \lambda ; x} \circ \exp _{\Psi_{i}^{-1}(x)}$ falls under Remark 7 and hence this expression is indeed bounded by a constant times $C_{2} \lambda^{\gamma}$.

As immediate consequence we get the following statement.

Corollary 12 Let $\left(\bar{\Psi}_{j}, \bar{U}_{j}\right)_{j \in J}$ be another finite atlas with subordinate partition of unity $\left(\bar{\phi}_{j}\right)_{j \in J}$. Then for $\gamma \leq 0$

$$
C^{\gamma}\left(M ;\left(\Psi_{i}, U_{i}\right), \phi_{i}\right)=C^{\gamma}\left(M ;\left(\bar{\Psi}_{j}, \bar{U}_{j}\right), \bar{\phi}_{j}\right)
$$

with equivalent norms.

\section{Regularity structures on manifolds}

Let $M$ be a $d$-dimensional Riemannian manifold without boundary. The two cases we are most interested in are

- $M$ is compact without boundary (i.e. closed)

- $M$ is an open bounded subset of $\mathbb{R}^{d}$ with induced Euclidean metric

We now give our definition of a regularity structure and a model on a manifold $M$. For concrete incarnations of these abstract definitions we refer the reader to Sect. 4 for the implementation of a first order "polynomial" structure; to Sect. 9 for a structure implementing "polynomials" of any order and right before Lemma 36 for the structure used for the parabolic Anderson model.

Definition 13 (Regularity structure) A regularity structure is a graded vector bundle $\mathcal{G}$ on $M$, with a finite grading $A=A(\mathcal{G}) \subset \mathbb{R}$. For $a \in A, \mathcal{G}_{a}$ denotes the vector bundle of homogeneity $a$; it is assumed to be finite dimensional. We denote the fiber at $p \in M$ by $\left.\mathcal{G}\right|_{p}$ and the fiber of homogeneity $a$ at $p$ by $\left.\mathcal{G}_{a}\right|_{p}$. For $p \in M,\left.\tau \in \mathcal{G}\right|_{p}$, $a \in A$ we write $\operatorname{proj}_{\left.\mathcal{G}_{a}\right|_{p}} \tau$ for the projection of $\tau$ onto $\left.\mathcal{G}_{a}\right|_{p}$. We assume that $\mathcal{G}$ comes equipped with a norm $\|$.$\| . We denote the fiber norm of the restriction to homogeneity$ $a$ by $\|\tau\|_{a}:=\left\|\operatorname{proj}_{\left.\mathcal{G}_{a}\right|_{p}} \tau\right\|$.

Definition 14 (Model) Let a collection of open sets $\mathcal{U}_{q} \subset M, q \in M$, with $q \in \mathcal{U}_{q}$, and linear maps

$$
\begin{aligned}
\Pi_{q}:\left.\mathcal{G}\right|_{q} & \rightarrow D^{\prime}\left(\mathcal{U}_{q}\right) \\
\Gamma_{p \leftarrow q}:\left.\mathcal{G}\right|_{q} & \left.\rightarrow \mathcal{G}\right|_{p},
\end{aligned}
$$

be given. We assume there is for every compactum $\mathcal{K} \subset M$ a constant $\delta_{\mathcal{K}}=$ $\delta_{\mathcal{K}}\left(\Pi, \Gamma,\left\{\mathcal{U}_{q}\right\}_{q}\right)>0$, such that $\Gamma_{p \leftarrow q}$ is defined for $p, q \in \mathcal{K}, d(p, q)<\delta_{\mathcal{K}}$ and 
for $q \in \mathcal{K},\left.\exp _{q}\right|_{B_{T_{p} M}\left(0_{p}, \delta_{\mathcal{K}}\right)}$ is a diffeomorphism and $\exp _{q}\left(B_{T_{p} M}\left(0_{p}, \delta_{\mathcal{K}}\right)\right) \subset \mathcal{U}_{q}$. Assume moreover $\Gamma_{p \leftarrow p}=$ id for every $p \in M$. Given $\beta \in \mathbb{R}$, we say that $(\Pi, \Gamma)$ is a model with transport precision $\beta$ if the following entity is finite for every compactum $\mathcal{K} \subset M$

$$
\begin{aligned}
\|\Pi, \Gamma\|_{\beta ; \mathcal{K}}:= & \sup _{p \in \mathcal{K}, \ell \in A(\mathcal{G}),\left.\tau \in \mathcal{G}_{\ell}\right|_{p}, \lambda \in(0,1], \varphi \in \mathcal{B}_{T_{p} M}^{r, \delta \mathcal{K}}} \frac{\left|\left\langle\Pi_{p} \tau, \varphi_{p}^{\lambda}\right\rangle\right|}{\lambda^{\ell}|| \tau||} \\
& +\sup _{p, q \in \mathcal{K}: d(p, q)<\delta_{\mathcal{K}} / 2,\left.\tau \in \mathcal{G}\right|_{q}, \lambda \in(0,1], \varphi \in \mathcal{B}_{T_{p} M}^{r, \delta \mathcal{K}} / 2} \frac{\left|\left\langle\Pi_{q} \tau-\Pi_{p} \Gamma_{p \leftarrow q} \tau, \varphi_{p}^{\lambda}\right\rangle\right|}{\lambda^{\beta}\|\tau\|} \\
& +\sup _{\ell, m \in A(\mathcal{G}) ; p, q \in \mathcal{K}, d(p, q)<\delta_{\mathcal{K}} ;\left.\tau \in \mathcal{G}_{\ell}\right|_{q}} \frac{\left\|\Gamma_{p \leftarrow q} \tau\right\|_{m}}{d(p, q)^{(\ell-m) \vee 0}\|\tau\|},
\end{aligned}
$$

where we recall that the set of test functions $\mathcal{B}_{T_{p} M}^{r, \delta}$ was defined in (3).

Remark 15 The additional restriction on distance and support in the second supremum are necessary, since otherwise the action of $\Pi_{q} \tau$ on $\varphi_{p}^{\lambda}$ might not be well-defined.

Remark 16 Note that the conditions on a model do not pin down the global regularity of $\Pi_{q} \tau$. Without loss of generality we will assume that $\Pi_{q} \tau \in C^{\alpha}\left(U_{q}\right)$ for all $q \in$ $M,\left.\tau \in \mathcal{G}\right|_{q}$ and $\alpha:=\min A(\mathcal{G})$.

Our definition of a regularity structure and a corresponding model are slightly more general than the original formulation by Hairer [9]. This extension is necessary to accommodate the "polynomial regularity structure", which will be constructed up to first order in Sect. 4 and up to any order in Sect. 9. Let us point out the key differences.

- Derivatives of functions on a general manifold $M$ can only be coordinate invariantly stored in a fibered space. Hence the regularity structure has to be a vector bundle and not a fixed vector space.

- For this reason there cannot be a fixed structure group $G$ in which the transport maps $\Gamma_{p \leftarrow q}$ take value.

- The transport maps $\Gamma_{p \leftarrow q}$ can also act "upwards", see Remark 83.

- The distributions $\Pi_{p} \tau$ as well as the transports $\Gamma_{p \leftarrow q}$ only make sense locally.

- The identities $\Gamma_{p \leftarrow q} \Gamma_{q \leftarrow r}=\Gamma_{p \leftarrow r}$ and $\Pi_{p} \Gamma_{p \leftarrow q}=\Pi_{q}$ do not hold. An approximate version of the latter is incorporated into the norm (transport precision $\beta$ ). The former is, in the flat case, used in an extension argument ([9, Proposition 3.31]), which we do not need here.

It turns out that the theory can handle these slight extensions. In particular the reconstruction theorem still holds, Theorem 23. Finally, we remark that our regularity structure does not include time and that the parabolic Anderson model will be treated by considering functions in time, valued in modelled distributions (Definition 18) on a manifold.

As in Lemma 8 we know how $\Pi_{p} \tau$ acts on a more general class of functions: 
Lemma 17 For a regularity structure $\mathcal{G}$ let be given a model $(\Pi, \Gamma)$ of transport precision $\beta$ with $\beta \geq \sup _{a \in A(\mathcal{G})}|a|$. Let $p \in \mathcal{K}$, a compactum in $M$. Let $\lambda \in(0,1]$ and let $\varphi$ satisfy the assumptions of Lemma 8 , with $\mathbb{R}^{d}$ replaced by $T_{p} M$ and the additional condition supp $\varphi \subset B_{T_{p} M}\left(0_{p}, \delta_{\mathcal{K}} / 4\right)$. Assume moreover that $B_{M}\left(p, \delta_{\mathcal{K}} / 2\right) \subset \mathcal{K}$ (which can always be achieved by making $\delta_{\mathcal{K}}$ smaller). Then for $\left.\tau \in \mathcal{G}_{\ell}\right|_{p}$

$$
\left|\left\langle\Pi_{p} \tau, \varphi \circ \exp _{p}^{-1}\right\rangle\right| \lesssim C_{\varphi}\|\Pi, \Gamma\|_{\beta, \mathcal{K}}\|\tau\| \lambda^{\ell}
$$

where $C_{\varphi}:=C(\varphi, \lambda, 0, N, r)$ is defined in Lemma 8.

Proof Let $O: \mathbb{R}^{d} \rightarrow T_{p} M$ be a linear isometry. Uniform estimates on $D^{k} \varphi$ on balls in $T_{p} M$ are equivalent to uniform estimates on $D^{k}(\varphi \circ O)$ on balls in $\mathbb{R}^{d}$. We can hence consider $\varphi$ as a function on $\mathbb{R}^{d}$.

Let $\phi_{z}, z \in \mathbb{Z}^{d}$, be a partition of unity of $\mathbb{R}^{d}$ such that $\operatorname{supp} \phi_{z} \subset B_{\mathbb{R}^{d}}(z, 1)$ and $\sup _{z \in \mathbb{Z}^{d}}\left\|\phi_{z}\right\|_{C^{r}}<\infty$. Let $\lambda \mathcal{K}:=\lambda \delta_{\mathcal{K}} / 4$. Define

$$
\varphi_{z, \lambda \mathcal{K}}:=\phi_{z}\left(\lambda_{\mathcal{K}}^{-1} \cdot\right) \varphi \quad z \in \mathbb{Z}^{d}
$$

so that

$$
\sum_{z \in \mathbb{Z}^{d}} \varphi_{z, \lambda \mathcal{K}}=\varphi
$$

Then supp $\varphi_{z, \lambda_{\mathcal{K}}} \subset B_{\mathbb{R}^{d}}\left(\lambda_{\mathcal{K}} z, \lambda_{\mathcal{K}}\right) \cap B_{\mathbb{R}^{d}}\left(0, \delta_{\mathcal{K}} / 4\right)$. Hence $\varphi_{z, \lambda \mathcal{K}} \equiv 0$ for $\left|\lambda_{\mathcal{K}} z\right| \geq$ $\delta_{\mathcal{K}} / 2$. Moreover

$$
\left\|D^{k} \varphi_{z, \lambda \mathcal{K}}\right\|_{\infty} \lesssim C_{\varphi} \lambda^{-d-k} \frac{1}{1+|z|^{N}}
$$

Then

$$
\begin{aligned}
& \left\langle\Pi_{p} \tau, \varphi \circ \exp _{p}^{-1}\right\rangle=\sum_{z \in \mathbb{Z}^{d}}\left\langle\Pi_{p} \tau, \varphi_{z, \lambda \mathcal{K}} \circ \exp _{p}^{-1}\right\rangle
\end{aligned}
$$

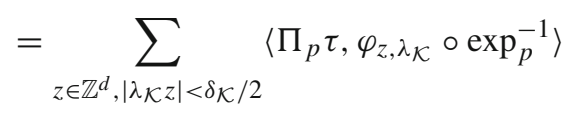

$$
\begin{aligned}
& =\sum_{z \in \mathbb{Z}^{d},\left|\lambda_{\mathcal{K}} z\right|<\delta_{\mathcal{K}} / 2}\left\langle\Pi_{\exp _{p}\left(\lambda_{\mathcal{K}} z\right)} \Gamma_{\exp _{p}\left(\lambda_{\mathcal{K}} z\right) \leftarrow p} \tau, \varphi_{z, \lambda_{\mathcal{K}}} \circ \exp _{p}^{-1}\right\rangle
\end{aligned}
$$

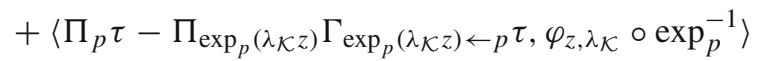

Note that in the sum $\left|\lambda_{\mathcal{K}} z\right|<\delta_{\mathcal{K}} / 2$. Hence, by assumption $q:=\exp _{p}\left(\lambda_{\mathcal{K}} z\right) \in \mathcal{K}$. Hence by definition of a model, $\left\|\Gamma_{p \leftarrow q} \tau\right\|_{m} \leq\|\Pi, \Gamma\|_{\beta, \mathcal{K}}\|\tau\| d(p, q)^{(\ell-m) \vee 0}=$ $\left\|\Pi, \Gamma||_{\beta, \mathcal{K}}\right\| \tau||\left|\lambda_{\mathcal{K}} z\right|^{(\ell-m) \vee 0}$, for $\left.\tau \in \mathcal{G}_{\ell}\right|_{q} \cdot{ }^{2}$ Then for those $z$

\footnotetext{
2 The particular choice of $\delta_{\mathcal{K}} / 4-, \delta_{\mathcal{K}} / 2$-balls in the assumptions is needed in order to be able to invoke the norm here.
} 


$$
\begin{aligned}
& \left|\left\langle\Pi_{\exp _{p}\left(\lambda_{\mathcal{K} z}\right)} \Gamma_{\exp _{p}\left(\lambda_{\mathcal{K}} z\right) \leftarrow p} \tau, \varphi_{z, \lambda_{\mathcal{K}}} \circ \exp _{p}^{-1}\right\rangle\right| \\
& \leq \sum_{n \leq \ell}\left|\left\langle\Pi_{\exp _{p}\left(\lambda_{\mathcal{K}} z\right)} \operatorname{proj}_{\mathcal{T}_{n}} \Gamma_{\exp _{p}\left(\lambda_{\mathcal{K}} z\right) \leftarrow p} \tau, \varphi_{z, \lambda_{\mathcal{K}}} \circ \exp _{p}^{-1}\right\rangle\right| \\
& +\sum_{n>\ell}\left|\left\langle\Pi_{\exp _{p}\left(\lambda_{\mathcal{K}} z\right)} \operatorname{proj}_{\mathcal{T}_{n}} \Gamma_{\exp _{p}\left(\lambda_{\mathcal{K}} z\right) \leftarrow p} \tau, \varphi_{z, \lambda \mathcal{K}} \circ \exp _{p}^{-1}\right\rangle\right| \\
& \lesssim C_{\varphi}\|\Pi, \Gamma\|_{\beta, \mathcal{K}}\|\tau\|\left(\sum_{n \leq \ell} \lambda^{n} \frac{1}{1+|z|^{N}}|\lambda z|^{\ell-n}+\sum_{n>\ell} \lambda^{n} \frac{1}{1+|z|^{N}}\right) .
\end{aligned}
$$

Moreover, the model being of transport precision $\beta$, we get

$$
\left|\left\langle\Pi_{p} \tau-\Pi_{\exp _{p}\left(\lambda_{\mathcal{K} z}\right)} \Gamma_{\exp _{p}\left(\lambda_{\mathcal{K}} z\right) \leftarrow p} \tau, \varphi_{z, \lambda_{\mathcal{K}}} \circ \exp _{p}^{-1}\right\rangle\right| \lesssim C_{\varphi}\|\Pi, \Gamma \mid\|_{\beta, \mathcal{K}}\|\tau\| \lambda^{\beta} \frac{1}{1+|z|^{N}}
$$

Combining,

$$
\begin{aligned}
& \left|\left\langle\Pi_{p} \tau, \varphi \circ \exp _{p}^{-1}\right\rangle\right| \\
& \quad \lesssim C_{\varphi}\|\Pi, \Gamma\|_{\beta, \mathcal{K}}\|\tau\| \sum_{z \in \mathbb{Z}^{d}}\left(\lambda^{\ell} \sum_{n \leq \ell} \frac{1}{1+|z|^{N}}|z|^{\ell-n}+\lambda^{\ell} \frac{1}{1+|z|^{N}} \cdot+\lambda^{\beta} \frac{1}{1+|z|^{N}}\right) \\
& \quad \lesssim C_{\varphi}\|\Pi, \Gamma\|_{\beta, \mathcal{K}}\|\tau\| \lambda^{\ell} .
\end{aligned}
$$

Definition 18 Let $\mathcal{G}$ be a regularity structure and $(\Pi, \Gamma)$ a model of precision $\beta \in \mathbb{R}$. Define for $\gamma>\sup _{\alpha \in A(\mathcal{G})}|\alpha|$ the space of modelled distributions

$\mathscr{D}^{\gamma}(M, \mathcal{G}):=\{f: M \rightarrow \mathcal{G}: f$ is a section of $\mathcal{G}$,

$\|f\|_{\mathscr{D}^{\gamma}(\mathcal{K}, \mathcal{G})}<\infty$ for all compacta $\left.\mathcal{K} \subset M\right\}$.

with

$$
\|f\|_{\mathscr{D} \gamma(\mathcal{K}, \mathcal{G})}:=\sum_{\ell<\gamma} \sup _{p \in \mathcal{K}}|f(p)|_{\ell}+\sup _{\ell<\gamma} \sup _{p, q \in \mathcal{K}, d(p, q)<\delta \mathcal{K}} \frac{\left|f(p)-\Gamma_{p \leftarrow q} f(q)\right|_{\ell}}{d(p, q)^{\gamma-\ell}} .
$$

Here $\delta_{\mathcal{K}}$ is the distance of points in $\mathcal{K}$ for which $\Gamma$ makes sense, see Definition 14. Note that the precision of transport $\beta$ plays no role here.

Remark 19 As usual for Hölder norms, for every compactum $\mathcal{K}$ an equivalent norm is obtained by replacing in the supremum, for any $\delta^{\prime} \in\left(0, \delta_{\mathcal{K}}\right]$, the condition $d(p, q)<$ $\delta_{\mathcal{K}}$ with the condition $d(p, q)<\delta^{\prime}$. 
Lemma 20 (Push-forward) Let $M, N$ be Riemannian manifolds and let $\Psi: M \rightarrow N$ be a diffeomorphism. Let $\mathcal{G}$ be a regularity structure on $M$ with model $(\Pi, \Gamma)$ with transport precision $\beta \in \mathbb{R}$. Define

$$
\begin{aligned}
\overline{\mathcal{U}}_{q} & :=\Psi\left(\mathcal{U}_{\Psi^{-1}(q)}\right), \\
\overline{\mathcal{G}}_{q} & :=\mathcal{G}_{\Psi^{-1}(q)}, \quad q \in N, \\
\bar{\Gamma}_{p \leftarrow q} & :=\Gamma_{\Psi^{-1}(p) \leftarrow \Psi^{-1}(q)}, \quad p, q \in N, \\
\bar{\Pi}_{q} \tau & :=\Psi_{*} \Pi_{\Psi^{-1}(q)} \tau, \quad q \in N,\left.\tau \in \overline{\mathcal{G}}\right|_{q} .
\end{aligned}
$$

Then, $\overline{\mathcal{G}}$ is a regularity structure on $N$ with grading $^{-} A=A$ and $(\bar{\Pi}, \bar{\Gamma})$ is a model with transport precision $\beta$. Moreover for every compactum $\mathcal{C} \subset N$ and all compacta $\mathcal{K} \subset \mathcal{C}$

1.

$$
\|\bar{\Pi}, \bar{\Gamma}\|_{\beta, \mathcal{K}} \lesssim \mathcal{C}\|\Pi, \Gamma\|_{\beta, \Psi^{-1}(\mathcal{K})}
$$

2. Let $f, f^{\prime} \in \mathscr{D}^{\gamma}(M, \mathcal{G})$ and define $\tilde{f}(x):=f\left(\Psi^{-1}(x)\right), \tilde{f}^{\prime}(x):=f^{\prime}\left(\Psi^{-1}(x)\right)$. Then $\tilde{f}, \tilde{f}^{\prime} \in \mathscr{D}^{\gamma}(\Psi(\mathcal{U}), \mathcal{G})$ and

$$
\begin{aligned}
\|\bar{f}\|_{\mathscr{D} \gamma(\mathcal{K}, \mathcal{G})} & \lesssim \mathcal{C}\|f\|_{\mathscr{D} \gamma\left(\Psi^{-1}(\mathcal{K}), \mathcal{G}\right)} \\
\left\|\tilde{f}-\tilde{f}^{\prime}\right\|_{\mathscr{D} \gamma(\mathcal{K}, \mathcal{G})} & \lesssim \mathcal{C}\left\|f-f^{\prime}\right\|_{\mathscr{D} \gamma\left(\Psi^{-1}(\mathcal{K}), \mathcal{G}\right)}
\end{aligned}
$$

Proof Since $\Psi$ has derivative uniformly bounded below and above for every compactum $\mathcal{K} \subset \mathcal{C}$, one can choose for every compactum $\mathcal{K}$ a constant $\bar{\delta}_{\mathcal{K}}$ as in the definition of a model, such that $\bar{\Gamma}_{p \leftarrow q}$ is well-defined for $p, q \in \mathcal{K}$ and $d(p, q)<\delta_{\mathcal{K}}$ as well as $\exp _{q}^{N}\left(B_{T_{q} M}\left(0_{q}, \delta_{\mathcal{K}}\right) \subset \overline{\mathcal{U}}_{q}\right.$. Here $\exp ^{N}$ denotes the exponential map on $N$.

1. Let $q \in \mathcal{K} \subset N$ and $\left.\tau \in \tilde{\mathcal{G}}_{a}\right|_{q}$ and $\varphi \in \mathcal{B}_{T_{q} M}^{r, \delta_{\mathcal{K}}}$

$$
\begin{aligned}
\left|\left\langle\tilde{\Pi}_{q} \tau, \varphi_{q}^{\lambda}\right\rangle\right| & =\left|\left\langle\Psi_{*}\left(\Pi_{\Psi^{-1}(q)} \tau\right), \varphi_{q}^{\lambda}\right\rangle\right| \\
& =\left|\left\langle\Pi_{\Psi^{-1}(q)} \tau, \varphi_{q}^{\lambda} \circ \Psi\right\rangle\right| \\
& =\left|\left\langle\Pi_{\Psi^{-1}(q)} \tau, \varphi_{q}^{\lambda} \circ \Psi \circ \exp _{q} \circ \exp _{q}^{-1}\right\rangle\right| \\
& \lesssim \mathcal{C}|| \Pi, \Gamma \|_{\beta, \Psi^{-1}(\mathcal{K})}|| \tau|| \lambda^{a},
\end{aligned}
$$

since $\varphi_{q}^{\lambda} \circ \Psi \circ \exp _{q}$ falls under Remark 7. For $p, q \in \mathcal{K} \subset N$ with $d(p, q)<\delta_{\mathcal{K}}$ and $\left.\tau \in \tilde{\mathcal{G}}\right|_{q}$, we have

$$
\begin{aligned}
\left|\left\langle\tilde{\Pi}_{q} \tau-\tilde{\Pi}_{p} \tilde{\Gamma}_{x \leftarrow y} \tau, \varphi_{p}^{\lambda}\right\rangle\right| & =\left|\left\langle\Psi_{*}\left(\Pi_{\Psi^{-1}(q)} \tau-\Pi_{\Psi^{-1}(p)} \Gamma_{\Psi^{-1}(p) \leftarrow \Psi^{-1}(q)} \tau\right), \varphi_{p}^{\lambda}\right\rangle\right| \\
& =\left|\left\langle\Pi_{\Psi^{-1}(q)} \tau-\Pi_{\Psi^{-1}(p)} \Gamma_{\Psi^{-1}(p) \leftarrow \Psi^{-1}(q)} \tau, \varphi_{p}^{\lambda} \circ \Psi\right\rangle\right| \\
& =\left|\left\langle\Pi_{\Psi^{-1}(q)} \tau-\Pi_{\Psi^{-1}(p)} \Gamma_{\Psi^{-1}(p) \leftarrow \Psi^{-1}(q)} \tau, \varphi_{p}^{\lambda} \circ \Psi \circ \exp _{p} \circ \exp _{p}^{-1}\right\rangle\right| \\
& \lesssim \mathcal{C}\|\Pi, \Gamma\|_{\beta, \Psi^{-1}(\mathcal{K})}\|\tau\| \lambda^{\beta},
\end{aligned}
$$


$\overline{\text { again by Remark 7. Finally for } p, q \in \mathcal{K} \subset N \text { with } d(p, q)<\delta_{\mathcal{K}} \text { and }\left.\tau \in \mathcal{G}_{a}\right|_{q} \text {, we }}$ have

$$
\left|\bar{\Gamma}_{p \leftarrow q} \tau\right|=\left|\Gamma_{\Psi^{-1}(p) \leftarrow \Psi^{-1}(q)} \tau\right|_{m} \lesssim \mathcal{C}\|\Pi, \Gamma\|_{\beta, \Psi^{-1}(\mathcal{K})}\|\tau\| d(p, q)^{(\ell-m) \vee 0} .
$$

2. Let $p, q \in \mathcal{K} \subset N$ then

$$
\begin{aligned}
\left\|\bar{f}(q)-\bar{\Gamma}_{p \leftarrow q} \bar{f}(q)\right\|_{m} & =\left\|f\left(\Psi^{-1}(q)\right)-\Gamma_{\Psi^{-1}(p) \leftarrow \Psi^{-1}(q)} f\left(\Psi^{-1}(q)\right)\right\|_{m} \\
& \lesssim \mathcal{C}\|f\|_{\mathcal{D} \gamma\left(\Psi^{-1}(\mathcal{K}), \mathcal{G}\right)} d\left(\Psi^{-1}(p), \Psi^{-1}(q)\right)^{\gamma-m} \\
& \lesssim \mathcal{C}\|f\|_{\mathcal{D}^{\gamma}\left(\Psi^{-1}(\mathcal{K}), \mathcal{G}\right)} d(p, q)^{\gamma-m}
\end{aligned}
$$

and similarly for the distance of two modelled distributions.

Lemma 21 (Reconstruction for $M \subset \mathbb{R}^{d}$ ) Let $\mathcal{G}$ be a regularity structure on $M$, an open connected subset of $\mathbb{R}^{d}$. Let $(\Pi, \Gamma)$ be a model with precision $\beta \in \mathbb{R}$. Let $\gamma>0$ and assume $\beta \geq \gamma$. Denote $\alpha:=\inf A$. Assume either that $\alpha<0$, or that $\alpha=0$ and that the lowest homogeneity in $\mathcal{G}$ is given by the constant distribution (of the polynomial regularity structure of Sect. 4).

For every $f \in \mathscr{D}^{\gamma}(M, \mathcal{G})$ there exists a unique $\mathcal{R} f \in C^{\alpha}(M)$ such that for every compactum $\mathcal{K} \subset M$

$$
\left|\left\langle\mathcal{R} f-\Pi_{x} f(x), \varphi_{x}^{\lambda}\right\rangle\right| \lesssim \lambda^{\gamma}\|\Pi, \Gamma\|_{\beta ; \overline{\mathcal{K}}}\|f\|_{\gamma ; \overline{\mathcal{K}}}
$$

Here $\varphi \in \mathcal{B}_{T_{p} M}^{r, \delta_{\mathcal{K}}}, r=-[\alpha]$, (so that the action of $\Pi_{x} f(x)$ is well-defined) and $\overline{\mathcal{K}}$ is the closure of the $\delta_{\mathcal{K}}$ thickening of $\mathcal{K}$.

Remark 22 Uniqueness actually holds in the class of operators $\mathcal{R}$ that satisfy (5) with $\gamma$ replaced by any $\theta>0$.

\section{Proof Existence}

We will apply [9, Proposition 3.25]. ${ }^{3}$ This Proposition is formulated for $\mathbb{R}^{d}$, but the statement is local and also holds for $M \subset \mathbb{R}^{d}$. So we have to verify for $\zeta_{x}:=\Pi_{x} f(x)$

$$
\begin{aligned}
\left|\left\langle\varphi_{x}^{n}, \zeta_{x}-\zeta_{y}\right\rangle\right| & \leq C_{1}|x-y|^{\gamma-\alpha} 2^{-n d / 2-\alpha n} \\
\left|\left\langle\varphi_{x}^{n}, \zeta_{x}\right\rangle\right| & \leq C_{2} 2^{-\alpha n-n d / 2}
\end{aligned}
$$

uniformly over $x, y \in \overline{\mathcal{K}}, n \geq n_{0}, n_{0}=\log _{2}\left(\delta_{\overline{\mathcal{K}}}\right) \vee 0$ and $2^{-n} \leq|x-y| \leq \delta_{\overline{\mathcal{K}}}$. In $[9$, Proposition 3.25] the upper bound 1 is chosen on $|x-y|$, but any upper bound works, so we chose $\delta_{\overline{\mathcal{K}}}$, since we need $\Gamma_{x \leftarrow y}$ to be well-defined.

Here

$$
\varphi_{x}^{n}:=2^{n d / 2} \varphi\left(2^{n}(\cdot-x)\right)
$$

\footnotetext{
3 Compare also [10, Theorem 2.10] for a concise presentation of the (wavelet) techniques involved in its proof. 
and $\varphi$ is a scaling function for a wavelet basis of regularity $r>|\alpha|$. We have chosen $n_{0}$ also such that for $n \geq n_{0}$ and $x \in \overline{\mathcal{K}},\left.\tau \in \mathcal{G}\right|_{x}$ the expression $\left\langle\Pi_{x} \tau, \varphi_{x}^{n}\right\rangle$ is welldefined. First, (7) follows from the fact that $\alpha$ is the lowest homogeneity in $A(\mathcal{G})$ (note that $\varphi_{x}^{n}$ is scaled to preserve the $L^{2}$-norm, whereas the scaling in the definition of a model preserves the $L^{1}$-norm).

Now

$$
\begin{aligned}
\left\langle\varphi_{x}^{n}, \zeta_{x}-\zeta_{y}\right\rangle & =\left\langle\varphi_{x}^{n}, \Pi_{x} f(x)-\Pi_{y} f(y)\right\rangle \\
& =\left\langle\varphi_{x}^{n}, \Pi_{x}\left[f(x)-\Gamma_{x \leftarrow y} f(y)\right]\right\rangle+\left\langle\varphi_{x}^{n}, \Pi_{x} \Gamma_{x \leftarrow y} f(y)-\Pi_{y} f(y)\right\rangle .
\end{aligned}
$$

We bound the first term as

$$
\begin{aligned}
\left|\left\langle\varphi_{x}^{n}, \Pi_{x}\left[f(x)-\Gamma_{x \leftarrow y} f(y)\right]\right\rangle\right| & \leq \sum_{a}\left|\left\langle\varphi_{x}^{n}, \Pi_{x} \operatorname{proj}_{\mathcal{G}_{a}}\left[f(x)-\Gamma_{x \leftarrow y} f(y)\right]\right\rangle\right| \\
& \lesssim\|\Pi, \Gamma\|_{\beta, \overline{\mathcal{K}}}\|f\|_{\mathscr{D} \gamma(\overline{\mathcal{K}}, \mathcal{G})} \sum_{a} 2^{-n a-n d / 2}|x-y|^{\gamma-a} \\
& \lesssim\|\Pi, \Gamma\|_{\beta, \overline{\mathcal{K}}}\|f\|_{\mathscr{D} \gamma(\overline{\mathcal{K}}, \mathcal{G})} 2^{-n \alpha-n d / 2}|x-y|^{\gamma-\alpha},
\end{aligned}
$$

since $2^{-n} \leq|x-y|$. The second term is bounded as

$$
\begin{aligned}
\left|\left\langle\varphi_{x}^{n}, \Pi_{x} \Gamma_{x \leftarrow y} f(y)-\Pi_{y} f(y)\right\rangle\right| & \lesssim\|\Pi, \Gamma\|_{\beta, \overline{\mathcal{K}}}\|f\|_{\mathscr{D} \gamma(\overline{\mathcal{K}}, \mathcal{G})} 2^{-n \beta-n d / 2} \\
& =\|\Pi, \Gamma\|_{\beta, \overline{\mathcal{K}}}\|f\|_{\mathscr{D} \gamma}\left(\overline{\mathcal{K}, \mathcal{G})} 2^{-n \alpha-n d / 2-n(\beta-\alpha)}\right. \\
& \lesssim\|\Pi, \Gamma\|\left\|_{\beta, \overline{\mathcal{K}}}\right\| f \|_{\mathscr{D}^{\gamma}(\overline{\mathcal{K}}, \mathcal{G})} 2^{-n \alpha-n d / 2}|x-y|^{\beta-\alpha} \\
& \lesssim\|\Pi, \Gamma\|_{\beta, \overline{\mathcal{K}}}\|f\|_{\mathscr{D} \gamma(\overline{\mathcal{K}}, \mathcal{G})} 2^{-n \alpha-n d / 2}|x-y|^{\gamma-\alpha},
\end{aligned}
$$

where we again used the assumption $2^{-n} \leq|x-y|$. This proves (6) and an application of [9, Proposition 3.25] gives the existence of $\mathcal{R} f$ satisfying the bound (5).

The preceding argument is valid for $\alpha<0$. For $\alpha=0$, one can run the argument for some $\alpha^{\prime}<0$ and get unique existence of $\mathcal{R} f \in C^{\alpha^{\prime}}$ with the claimed properties. In Corollary 24 below it is shown that actually $\mathcal{R} f \in C^{0}$.

\section{Uniqueness}

Uniqueness follows exactly as in [9, Section 3].

Lemma 23 (Reconstruction for $M$ a closed Riemannian manifold) Let $M$ be a closed Riemannian manifold with regularity structure $\mathcal{G}$ and $(\Pi, \Gamma)$ a model with transport precision $\beta \in \mathbb{R}$. Let $\gamma>0$, and $f \in \mathscr{D}^{\gamma}(M, \mathcal{G})$ and assume $\beta \geq \gamma$.

Denote $\alpha:=\inf A$. Assume either that $\alpha<0$ or that $\alpha=0$ and that the lowest homogeneity in $\mathcal{G}$ is given by the constant distribution (of the polynomial regularity structure).

Then, there exists a unique distribution $\mathcal{R} f \in C^{\alpha}(M)$ such that

$$
\left|\left\langle\mathcal{R} f-\Pi_{p} f(p), \varphi_{p}^{\lambda}\right\rangle\right| \lesssim \lambda^{\gamma}\|\Pi, \Gamma\|_{\beta ; M}\|f\|_{\mathcal{D} \gamma(M, \mathcal{G})},
$$

for $p \in M, \varphi \in \mathcal{B}_{T_{p} M}^{r, \delta_{M}}, r=-[\alpha]$ 
Proof By a cutting up procedure, it is enough to show (8) for $\varphi \in \mathcal{B}_{T_{p} M}^{r, \delta^{\prime}}$ with $\delta^{\prime} \in$ $\left(0, \delta_{M}\right]$ to be chosen.

Let $\left(\Psi_{i}, \mathcal{U}_{i}\right)_{i \in I}$ a finite atlas with subordinate partition of unity $\left(\phi_{i}\right)_{i \in I}$. On each chart, we push-forward the regularity structure, model and $f$ to $\Psi_{i}\left(\mathcal{U}_{i}\right)$, with corresponding reconstruction operation $\tilde{\mathcal{R}}_{i}$, model $\tilde{\Pi}_{i}$ and modelled distribution $\tilde{f}_{i}$. For each $i \in I$, fix a compactum $\mathcal{K}_{i} \subset \mathcal{U}_{i}$ such that $\operatorname{supp} \phi_{i}$ is strictly contained in $\mathcal{K}_{i}$. By Lemma 20,

$$
\begin{aligned}
& \left\|\tilde{f}_{i}\right\|_{\mathscr{D}^{\gamma}\left(\Psi_{i}\left(\mathcal{K}_{i}\right), \mathcal{G}\right)} \lesssim\|f\|_{\mathscr{D}^{\gamma}(M, \mathcal{G})} \\
& \left\|\tilde{f}_{i}-f_{i}^{\prime}\right\|_{\mathscr{D} \gamma\left(\Psi_{i}\left(\mathcal{K}_{i}\right), \mathcal{G}\right)} \lesssim\left\|f-f^{\prime}\right\|_{\mathscr{D} \gamma(M, \mathcal{G})} \\
& \left\|\tilde{\Pi}_{i}, \Gamma \Pi_{i}\right\|_{\beta, \Psi_{i}\left(\mathcal{K}_{i}\right)} \lesssim\|\Pi, \Gamma\|_{\beta, M} .
\end{aligned}
$$

Now reconstruct in each coordinate chart as $\tilde{T}_{i}:=\tilde{\mathcal{R}}_{i} \tilde{f}_{i}$ using Theorem 21 . Define $\mathcal{R} f:=\sum_{i \in I} \phi_{i}\left(\Psi_{i}^{-1}\right)_{*} \tilde{T}_{i}$. Then

$$
\begin{aligned}
\left\langle\mathcal{R} f-\Pi_{p} f(p), \varphi_{p}^{\lambda}\right\rangle & =\sum_{i}\left\langle\phi_{i}\left(\left(\Psi_{i}^{-1}\right)_{*} T_{i}-\Pi_{p} f(p)\right), \varphi_{p}^{\lambda}\right\rangle \\
& =\sum_{i}\left\langle\left(\Psi_{i}^{-1}\right)_{*} T_{i}-\Pi_{p} f(p), \phi_{i} \varphi_{p}^{\lambda}\right\rangle \\
& =\sum_{i}\left\langle T_{i}-\left(\Psi_{i}\right)_{*}\left(\Pi_{p} f(p)\right),\left(\phi_{i} \varphi_{p}^{\lambda}\right) \circ \Psi_{i}^{-1}\right\rangle
\end{aligned}
$$

If $p \notin \mathcal{U}_{i}$, we want the summand to vanish. So let $\delta^{\prime}:=\min _{i} d\left(\operatorname{supp} \phi_{i}, \partial \mathcal{U}_{i}\right)$. Then for $\varphi \in \mathcal{B}_{T_{p} M}^{r, \delta^{\prime}}, \lambda \in(0,1]$, we have $\phi_{i} \varphi_{p}^{\lambda} \neq 0$ implies $p \in \mathcal{U}_{i}$. Hence, if $p \notin \mathcal{U}_{i}$, we have $\phi_{i} \varphi_{p}^{\lambda}=0$, so the summand vanishes.

Otherwise, with $z:=\Psi_{i}(p)$

$$
\begin{aligned}
\left|\left\langle T_{i}-\left(\Psi_{i}\right)_{*}\left(\Pi_{p} f(p)\right),\left(\phi_{i} \varphi_{p}^{\lambda}\right) \circ \Psi_{i}^{-1}\right\rangle\right| \\
\quad=\left|\left\langle T_{i}-\left(\Psi_{i}\right)_{*}\left(\Pi_{p} f(p)\right),\left(\phi_{i} \lambda^{-d} \varphi\left(\lambda^{-1} \exp _{p}^{-1}(\cdot)\right)\right) \circ \Psi_{i}^{-1}\right\rangle\right| \\
\quad=\left|\left\langle T_{i}-\bar{\Pi}_{z} f(z),\left(\phi_{i} \lambda^{-d} \varphi\left(\lambda^{-1} \exp _{p}^{-1}(\cdot)\right)\right) \circ \Psi_{i}^{-1}\right\rangle\right| \\
\quad \lesssim\left\|\tilde{\Pi}_{i}, \tilde{\Gamma}_{i}\right\|_{\beta, \Psi_{i}\left(\mathcal{U}_{i}\right)}\|\tilde{f}\|_{\mathcal{D} \gamma\left(\Psi_{i}\left(\mathcal{U}_{i}\right), \tilde{\mathcal{G}}_{i}\right)} \lambda^{\gamma} \\
\quad \lesssim\left\|\Pi_{i}, \Gamma_{i}\right\|_{\beta, M}\|f\|_{\mathcal{D} \gamma(M, \mathcal{G})} \lambda^{\gamma},
\end{aligned}
$$

since $\left(\phi_{i} \lambda^{-d} \varphi\left(\lambda^{-1} \exp _{p}^{-1}(\cdot)\right)\right) \circ \Psi_{i}^{-1}$ falls under Remark 7 around $z$. Summing over $i$ gives (8).

Corollary 24 In the setting of the previous theorem, assume that the lowest homogeneity in $\mathcal{G}$ is 0 and that it is given by the constant (as in the polynomial regularity structure of Sect. 4). Then $\mathcal{R} f$ is given by projection onto that homogeneity, i.e.

$$
(\mathcal{R} f)(p)=f_{0}(p) .
$$


Proof Define $\tilde{\mathcal{R}} f(p):=f_{0}(p)$, then

$$
\left|\left\langle\tilde{\mathcal{R}} f(\cdot)-\Pi_{p} f(p)(\cdot), \varphi_{p}^{\lambda}\right\rangle\right|=\left|\left\langle\tilde{f}_{0}(\cdot)-f_{0}(p), \varphi_{p}^{\lambda}\right\rangle\right|+\left|\left\langle\sum_{\ell>0} \Pi_{p} \operatorname{proj}_{\left.\mathcal{G}_{\ell}\right|_{p}} f(p), \varphi_{p}^{\lambda}\right\rangle\right| .
$$

Recall that the projection proj is defined in Definition 13. The last term is of bounded by a constant times $\lambda^{\eta}$, where $\eta$ is the smallest homogeneity strictly larger than 0 .

For the second to last term we first write

$$
f_{0}(\cdot)-f_{0}(p)=\left(f(\cdot)-\Gamma_{. \leftarrow p} f(p)+\Gamma_{. \leftarrow p} \operatorname{proj}_{\mathcal{G}_{>0 \mid p}} f(p)\right)_{0} .
$$

Now, since $f \in \mathcal{D}^{\gamma}$,

$$
\left(f(\cdot)-\Gamma_{\cdot \leftarrow p} f(p)\right)_{0} \lesssim d(\cdot, p)^{\gamma} .
$$

By the properties of a model

$$
\left|\Gamma \cdot \leftarrow p \operatorname{proj}_{\mathcal{G}_{>0 \mid p}} f(p)\right|_{0} \lesssim d(\cdot, p)^{\eta}
$$

Hence $\left|f_{0}(\cdot)-f_{0}(p)\right| \lesssim d(\cdot, p)^{\eta \wedge \gamma}$ and then

$$
\left|\left\langle\tilde{f}_{0}(\cdot)-f_{0}(p), \varphi_{p}^{\lambda}\right\rangle\right| \lesssim \lambda^{\eta \wedge \gamma}
$$

Hence, by Remark 22, $\tilde{\mathcal{R}}=\mathcal{R}$.

We want to apply the Lemma 23 to the terms in the heat kernel asymptotics (Theorem 43). The problem is that their support will be of order 1 (and not of order $\lambda$ as for $\varphi_{x}^{\lambda}$ ). Hence we need the following refinement which is similar to Lemma 8 .

Lemma 25 In the setting of Lemma 23, let $\varphi$ satisfy the assumptions of Lemma 8 with the additional condition $\operatorname{supp} \varphi \subset B_{T_{p} M}\left(0_{p}, \delta_{\mathcal{K}} / 4\right)$. Then

$$
\left|\left\langle\mathcal{R} f-\Pi_{p} f(p), \varphi \circ \exp _{p}^{-1}\right)\right\rangle \mid \lesssim C_{\varphi} \lambda^{\gamma}\|\Pi\|_{\beta ; M}\|f\|_{\mathscr{D} \gamma(M, \mathcal{G})}
$$

where $C_{\varphi}:=C(\varphi, \lambda, 0, N, r)$ is defined in Lemma 8.

Proof Let $\varphi_{z, \tilde{\lambda}}$ be given as in the Proof of Lemma 17 with $\mathcal{K}:=M$.

Recall $\lambda_{M}:=\lambda \delta_{M} / 4$, and that $\operatorname{supp} \varphi_{z, \lambda_{M}} \subset B_{\mathbb{R}^{d}}\left(\lambda_{M} z, \lambda_{M}\right) \cap B_{\mathbb{R}^{d}}\left(0, \delta_{M} / 4\right)$. Hence $\varphi_{z, \lambda_{M}} \equiv 0$ for $\left|\lambda_{M} z\right| \geq \delta_{M} / 2$. Then with $\zeta_{r}:=\mathcal{R} f-\Pi_{r} f(r)$

$$
\begin{aligned}
\left\langle\zeta_{p}, \varphi \circ \exp _{p}^{-1}\right\rangle & =\sum_{z \in \mathbb{Z}^{d}}\left\langle\zeta_{p}, \varphi_{z, \tilde{\lambda}} \circ \exp _{p}^{-1}\right\rangle \\
& =\sum_{z \in \mathbb{Z}^{d},\left|\lambda_{M} z\right|<\delta_{M} / 2}\left\langle\zeta_{p}, \varphi_{z, \tilde{\lambda}} \circ \exp _{p}^{-1}\right\rangle
\end{aligned}
$$




$$
\begin{aligned}
& =\sum_{z \in \mathbb{Z}^{d},\left|\lambda_{M} z\right|<\delta_{M} / 2}\left[\left\langle\zeta_{\exp _{p}\left(\lambda_{M} z\right)}, \varphi_{z, \tilde{\lambda}} \circ \exp _{p}^{-1}\right\rangle\right. \\
& \left.\quad+\left\langle\zeta_{p}-\zeta_{\exp _{p}\left(\lambda_{M} z\right)}, \varphi_{z, \tilde{\lambda}} \circ \exp _{p}^{-1}\right\rangle\right] .
\end{aligned}
$$

Note that in the sum $\left|\lambda_{M} z\right|<\delta_{M} / 2$. Hence $\exp _{p}\left(\lambda_{M} z\right) \in M$ is well-defined. Now the first summand can be written as

$\left\langle\zeta_{\exp _{p}\left(\lambda_{M} z\right)}, \varphi_{z, \lambda_{M}} \circ \exp _{p}^{-1}\right\rangle=\left\langle\zeta_{\exp _{p}\left(\lambda_{M} z\right)}, \varphi_{z, \lambda_{M}} \circ \exp _{p}^{-1} \circ \exp _{\exp _{p}\left(\lambda_{M} z\right)} \circ \exp _{\exp _{p}\left(\lambda_{M} z\right)}^{-1}\right\rangle$

Applying Remark 7 to $\varphi_{z, \lambda_{M}} \circ \exp _{p}^{-1} \circ \exp _{\exp _{p}\left(\lambda_{M} z\right)}$ and (8), this is bounded by a

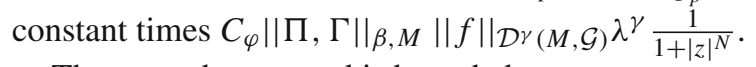

The second summand is bounded as

$$
\begin{aligned}
& \left|\left\langle\zeta_{p}-\zeta_{\exp _{p}(z)}, \varphi_{z, \lambda_{M}} \circ \exp _{p}\right\rangle\right|=\left|\left\langle\Pi_{\exp _{p}(z)} f\left(\exp _{p}(z)\right)-\Pi_{p} f(x), \varphi_{z, \lambda_{M}} \circ \exp _{p}\right\rangle\right| \\
& \leq\left|\left\langle\Pi_{\exp _{p}(z)}\left(f\left(\exp _{p}(z)\right)-\Gamma_{\exp _{p}(z) \leftarrow x} f(x)\right), \varphi_{z, \lambda_{M}} \circ \exp _{p}\right\rangle\right| \\
& \quad+\left|\left\langle\Pi_{\exp _{p}(z)} \Gamma_{\exp _{p}(z) \leftarrow x} f(x)-\Pi_{p} f(x), \varphi_{z, \lambda_{M}} \circ \exp _{p}\right\rangle\right| \\
& \quad \lesssim C_{\varphi}\|\Pi, \Gamma\|_{\beta, M}\|f\|_{\mathcal{D} \gamma(M, \mathcal{G})}\left(\sum_{\ell} \lambda^{\ell}\left|\lambda_{M} z\right|^{\gamma-\ell}+\lambda^{\beta}\right) \frac{1}{1+|z|^{N}} .
\end{aligned}
$$

Hence

$$
\begin{aligned}
& \left|\left\langle\zeta_{p}, \varphi \circ \exp _{p}^{-1}\right\rangle\right| \lesssim C_{\varphi}\|\Pi, \Gamma\|_{\beta, M}\|f\|_{\mathcal{D}^{\gamma}(M, \mathcal{G})}\left(\sum_{z \in \mathbb{Z}^{d}} \lambda^{\gamma} \frac{1}{1+|z|^{N}}\right. \\
& \left.\quad+\sum_{z \in \mathbb{Z}^{d}}\left(\sum_{a} \lambda^{a}\left|\lambda_{M} z\right|^{\gamma-a}+\lambda^{\beta}\right) \frac{1}{1+|z|^{N}}\right) \\
& \lesssim C_{\varphi}\|\Pi, \Gamma\|_{\beta, M}\|f\|_{\mathcal{D}^{\gamma}(M, \mathcal{G})}\left(\lambda^{\gamma}+\lambda^{\beta}+\lambda^{\gamma} \sum_{z \in \mathbb{Z}^{d}} \frac{1}{1+|z|^{N}}|z|^{\gamma+\left|\inf _{a \in A} a\right|}\right) \\
& \lesssim C_{\varphi}\|\Pi, \Gamma\|_{\beta, M}\|f\|_{\mathcal{D} \gamma(M, \mathcal{G})^{\gamma}} \lambda^{\gamma}
\end{aligned}
$$

for $N>d+\gamma+\left|\inf _{a \in A} a\right|$.

\section{Linear "polynomials" on a Riemannian manifold}

The regularity structure for linear "polynomials" on the Riemannian manifold $M$ will be built on the vector bundle $(M \times \mathbb{R}) \oplus T^{*} M$. For readability introduce the symbol 1 and decree that it forms a basis for $\mathbb{R}$. Define the graded vector bundle

$$
\mathcal{T}:=(M \times \mathbb{R} \mathbf{1}) \oplus T^{*} M,
$$


with grading $A(\mathcal{T})=\{0,1\}$. For $q \in M$ let $\left.\mathcal{T}\right|_{q}=\operatorname{span}\{\mathbf{1}\} \oplus T_{q}^{*} M$ be the fiber at $q$. A generic element of $\mathcal{T}_{q}$ will be written as

$$
1 a+\omega,
$$

with $a \in \mathbb{R}, \omega \in T_{q}^{*} M$. Let $\mathcal{U}_{q}:=B_{M}(q, \delta)$, where $\delta$ is the radius of injectivity of $M$. Define the linear map $\Pi_{q}: \mathcal{T}_{q} \rightarrow D^{\prime}\left(\mathcal{U}_{q}\right)$ as

$$
\begin{array}{lll}
\left(\Pi_{q} \mathbf{1}\right)(z)=1 & & z \in \mathcal{U}_{q} \\
\left(\Pi_{q} \omega\right)(z)=\omega \exp _{q}^{-1}(z), & & z \in \mathcal{U}_{q}, \omega \in T_{q}^{*} M .
\end{array}
$$

Note that, since $\mathbb{R} \mathbf{1}$ is a trivial fiber bundle, it is enough to specify it on the basis element 1 . This is not possible on $T^{*} M$. Note also that $\Pi_{q} \omega$ is chosen to have value 0 and differential $\omega$ at $q$.

Finally define the re-expansion maps $\Gamma_{p \leftarrow q}: \mathcal{T}_{q} \rightarrow \mathcal{T}_{p}$ as

$$
\begin{aligned}
& \Gamma_{p \leftarrow q} \mathbf{1}=\mathbf{1} \\
& \Gamma_{p \leftarrow q} \omega=\omega \exp _{q}^{-1}(p) \mathbf{1}+d\left[\omega \exp _{q}^{-1}\right](p),
\end{aligned}
$$

which is well-defined for $d(p, q)<\delta$. $\Pi$ and $\Gamma$ together form the polynomial model, where we take $\delta_{M}=\delta$ in Definition 14 .

Remark 26 Note that in Euclidean space with $\omega=d x_{i}$ we have

$$
\left(\Pi_{q} d x_{i}(z)=(z-q)_{i}\right.
$$

the classical linear polynomials "based" at $q$. Moreover

$$
\Gamma_{p \leftarrow q} d x_{i}=d x_{i}+(p-q)_{i},
$$

so we recover Hairer's definition [9].

The transport of $\omega \in T_{q}^{*} M$ is chosen such that $\Pi_{q} \omega$ and $\Pi_{p} \Gamma_{p \leftarrow q} \omega$ have, at $p$, the same value and the same first derivative. Our re-expansion is not exact, i.e. we do not have $\Pi_{q} \tau=\Pi_{p} \Gamma_{p \leftarrow q} \tau$, but we have the following.

Lemma 27 For $\omega \in T_{q}^{*} M$, uniformly for $d(p, q)$ bounded, $\ell=0,1,2$, and $V$ differential operators of order $\ell$

$$
\left|V\left(\left(\Pi_{q} \omega\right)(z)-\left(\Pi_{p} \Gamma_{p \leftarrow q} \omega\right)(z)\right)\right| \lesssim_{V} d(z, p)^{2-\ell} .
$$

\section{Proof Let}

$$
\begin{aligned}
f(z) & :=\left(\Pi_{q} \omega\right)(z) \\
g(z) & :=\left(\Pi_{p} \Gamma_{p \leftarrow q} \omega\right)(z) .
\end{aligned}
$$


By construction $f(p)=g(p),\left.d\right|_{p} f=\left.d\right|_{q} g$ and hence the statement follows from Taylor's theorem.

Remark 28 In the setting of the previous Lemma, not only $f(p)=g(p)$ but also $f(q)=g(q)$. Indeed, for two points $p, q \in M$, at distance smaller than the cut locus and $\omega_{q} \in T_{q}^{*} M$,

$$
\begin{aligned}
\Gamma_{p \leftarrow q}\left(\omega_{q}\right) & =\omega_{q}\left(\exp _{q}^{-1}(p)\right) \mathbf{1}+\left.d\right|_{p}\left[\omega_{q}\left(\exp _{q}^{-1}(\cdot)\right)\right] \\
& =\omega_{q}\left(\exp _{q}^{-1}(p)\right) \mathbf{1}+\left.\omega_{q} \circ d\right|_{p}\left(\exp _{q}^{-1}\right),
\end{aligned}
$$

where the tangent map satisfies indeed $\left.d\right|_{p}\left(\exp _{q}^{-1}\right): T_{p} M \rightarrow T_{q} M$. By definition,

$$
\Pi_{p}\left(\Gamma_{p \leftarrow q}\left(\omega_{q}\right)\right)=\omega_{q}\left(\exp _{q}^{-1}(p)\right)+\left.\omega_{q} \circ d\right|_{p}\left(\exp _{q}^{-1}\right) \circ \exp _{p}^{-1}
$$

does a priori disagree with $\Pi_{q}\left(\omega_{q}\right)=\omega_{q} \circ \exp _{q}^{-1}$, but at $p$. Let us set $v_{q}=\exp _{q}^{-1}(p)$ and $v_{p}=\exp _{p}^{-1}(q)$. The path $\gamma=\left(\exp _{q}\left((1-t) v_{q}\right)\right)_{0 \leq t \leq 1}$ is the unique path from $p$ to $q$, with length and speed both equal to $d(p, q)$, staying within the cut-locus from $y$, that is $\left(\exp _{p}\left(t v_{p}\right)\right)_{0 \leq t \leq 1}$ : in other words, for any $0 \leq t \leq 1$,

$$
\exp _{p}\left(t \exp _{p}^{-1}(q)\right)=\exp _{q}\left((1-t) \exp _{q}^{-1}(p)\right)
$$

Hence,

$$
\begin{aligned}
\left.d\right|_{p}\left(\exp _{q}^{-1}\right)\left(v_{p}\right) & =\left.\frac{d}{d t}\right|_{t=0} \exp _{q}^{-1}\left(\exp _{p}\left(t v_{p}\right)\right)=\left.\frac{d}{d t}\right|_{t=0} \exp _{q}^{-1}\left(\exp _{q}\left((1-t) v_{q}\right)\right) \\
& =-v_{q}
\end{aligned}
$$

and

$$
\Pi_{p}\left(\Gamma_{p \leftarrow q}\left(\omega_{q}\right)\right)(q)=\omega_{q}\left(v_{q}\right)+\left.\omega_{q} \circ d\right|_{p}\left(\exp _{q}^{-1}\right)\left(v_{p}\right)=0=\Pi_{q}\left(\omega_{q}\right)(q) .
$$

The next lemma follows from Lemma 27 and is shown in more generality in Theorem 91 .

Lemma 29 The above is a model of transport precision $\beta=2$.

As a sanity check for our construction, we mention the following lemma, which is almost immediate in the flat case (see [9, Lemma 2.12]). We will prove it in Sect. 9 in a more general setting.

Lemma 30 For $\gamma \in(1,2)$, a function $f: M \rightarrow \mathbb{R}$ is in $C^{\gamma}(M)$ if and only if there exists a section $\hat{f}(p)=f_{0}(p) \mathbf{1}+f_{1}(p) \in \mathscr{D}^{\gamma}(M, \mathcal{T})$ with $f_{0}(p)=f(p)$ and $f_{1}(p) \in T_{p}^{*} M$.

In that case: $f_{1}(p)=\left.d\right|_{p} f$. 


\section{The regularity structure for PAM on a manifold}

In the next five sections $M$ is a 2-dimensional closed manifold.

The regularity structure for PAM will be built on two copies of the vector bundle, $\left(M \times \mathbb{R}^{2}\right) \oplus T^{*} M$. We denote these two copies by $\mathcal{V}$ and $\mathcal{W}$. In order to distinguish the different elements of these bundles we introduce the symbols $\{\mathbf{1}, \boldsymbol{\Xi}, \mathcal{I}[\boldsymbol{\Xi}], \mathcal{I}[\boldsymbol{\Xi}] \boldsymbol{\Xi}\}$ and decree that they form a basis for $\mathbb{R}^{4}$. We then write

$$
\begin{aligned}
\mathcal{W} & =(M \times[\mathbb{R} \boldsymbol{1} \oplus \mathbb{R}[\boldsymbol{\Xi}]]) \oplus T^{*} M \text { and } \\
\mathcal{V} & =(M \times[\mathbb{R} \boldsymbol{\Xi} \oplus \mathbb{R}[\boldsymbol{\Xi}] \boldsymbol{\Xi}]) \oplus\left(\boldsymbol{\Xi} T^{*} M\right),
\end{aligned}
$$

where $\boldsymbol{\Xi} T^{*} M$ is simply another copy of $T^{*} M$. Formally we have, $\mathcal{V}=\mathcal{W} \boldsymbol{\Xi}$. As before we will let $\left.\mathcal{T}\right|_{p},\left.\mathcal{V}\right|_{p}$, and $\left.\mathcal{W}\right|_{p}$ denote the fibers of these bundles over $p \in M$.

The vector bundles $\mathcal{V}$ and $\mathcal{W}$ are graded, with gradings

$$
\begin{aligned}
A(\mathcal{V}) & :=\{\alpha, 2 \alpha+2, \alpha+1\} \\
A(\mathcal{W}) & :=\{0, \alpha+2,1\}
\end{aligned}
$$

for some $\alpha \in(-3 / 2,-1)$ corresponding to the regularity of the driving white noise $\xi$.

For $\beta \in A(\mathcal{V})$ (or $\beta \in A(\mathcal{W})$ ) recall (Definition 13) that $\operatorname{proj}_{\beta}: \mathcal{V} \rightarrow \mathcal{V}\left(\operatorname{proj}_{\beta}:\right.$ $\mathcal{W} \rightarrow \mathcal{W})$ is the projection taking an element to its $\beta$-component. To be concrete, generic elements $\left.\tau \in \mathcal{V}\right|_{p},\left.\tau^{\prime} \in \mathcal{W}\right|_{p}$ are of the form

$$
\begin{aligned}
\tau & =\boldsymbol{\Xi} a+\mathcal{I}[\mathbf{\Xi}] \mathbf{\Xi} b+\boldsymbol{\Xi} c \\
\tau^{\prime} & =\mathbf{1} d+\mathcal{I}[\mathbf{\Xi}] e+f,
\end{aligned}
$$

with $a, b, d, e \in \mathbb{R}, c, f \in T_{p}^{*} M$. And then for example

$$
\begin{aligned}
\operatorname{proj}_{\alpha} \tau & =\left.\boldsymbol{\Xi} a \in \mathcal{V}_{\alpha}\right|_{p} \\
\operatorname{proj}_{\alpha+2} \tau^{\prime} & =\left.\mathcal{I}[\boldsymbol{\Xi}] e \in \mathcal{W}_{\alpha+2}\right|_{p} .
\end{aligned}
$$

All the graded fibers have a canonical norm, where on the cotangent space we use the norm induced by the Riemannian metric. For $\beta \in A,\left.\tau \in \mathcal{V}\right|_{p}\left(\right.$ or $\left.\tau \in \mathcal{W}\right|_{p}$ ) we write as before, in a slight abuse of notation, $\|\tau\|_{\beta}:=\left\|\operatorname{proj}_{\beta} \tau\right\|$.

The model we shall use for the parabolic Anderson model will be time dependent, so we need slight extensions of our definitions.

Definition 31 For $\mathcal{G}=\mathcal{V}, \mathcal{W}$, assume we are given a family of models $\left(\Pi^{t}, \Gamma^{t}\right)$ on $M$ parametrized by $t \in[0, T]$. Define

$$
\|\Pi, \Gamma\|_{\beta, M, T}:=\sup _{t \leq T}\left\|\Pi^{t}, \Gamma^{t}\right\|_{\beta, M}
$$

where $\left\|\Pi^{t}, \Gamma^{t}\right\|_{\beta, M}$ is defined in Definition 14. Note that for fixed $t$, the model comes with a reconstruction operator (Theorem 23), which we shall denote $\mathcal{R}_{t}$. 
Definition 32 (Time-dependent modelled distributions) For $\mathcal{G}=\mathcal{V}, \mathcal{W}$, given a family of models $\left(\Pi^{t}, \Gamma^{t}\right)$ parametrized by $t \in[0, T]$, denote by $\mathscr{D}^{t, \gamma}(\mathcal{G})=\mathscr{D}^{t, \gamma}(M, \mathcal{G})$ the corresponding spaces of modelled distributions. That is, as defined in Definition 18,

$$
\|g\|_{\mathcal{D}^{t, \gamma}(M, \mathcal{G})}:=\sup _{p \in M} \sup _{\ell<\gamma}\|g(p)\|_{\ell}+\sup _{p, q \in M} \sup _{\ell<\gamma} \frac{\left\|g(p)-\Gamma_{p \leftarrow q}^{t} g(q)\right\|_{\ell}}{d(p, q)^{\gamma-\ell}}<\infty,
$$

For $\mathfrak{N}>0$, define the modified norm

$$
\begin{aligned}
\|g\|_{\mathcal{D}^{t, \gamma, \mathfrak{N}}(M, \mathcal{G})}:= & \sup _{p \in M} \sup _{\ell<\gamma}\|g(p)\|_{\ell}+\sup _{p, q \in M} \sup _{\ell<\gamma, \ell \neq \mu} \frac{\left\|g(p)-\Gamma_{p \leftarrow q}^{t} g(q)\right\|_{\ell}}{d(p, q)^{\gamma-\ell}} \\
& +\mathfrak{N} \sup _{p, q \in M} \frac{\left\|g(p)-\Gamma_{p \leftarrow q}^{t} g(q)\right\|_{\mu}}{d(p, q)^{\gamma-\mu}} .
\end{aligned}
$$

Here $\mu=\alpha$, if $\mathcal{G}=\mathcal{V}$ and $\mu=0$, if $\mathcal{G}=\mathcal{W}$.

Define $\mathscr{D}_{T}^{\gamma, \gamma_{0}}(M, \mathcal{G})$ to be the space of functions $f:[0, T] \times M \rightarrow \mathcal{G}$ with $f(t) \in \mathscr{D}^{t, \gamma}(M, \mathcal{G})$ and

$$
\|f\|_{\mathcal{D}_{T}^{\gamma, \gamma_{0}}(M, \mathcal{G})}:=\sup _{t \leq T}\|f(t)\|_{\mathcal{D}^{t, \gamma}(M, \mathcal{G})}+\sup _{p \in M, s, t \leq T} \frac{\|f(t, p)-f(s, p)\|_{v}}{|t-s|^{\gamma_{0}}}<\infty,
$$

where $v=\alpha$, if $\mathcal{G}=\mathcal{V}$ and $v=0$, if $\mathcal{G}=\mathcal{W}$. For $\mathfrak{N}>0$, define the modified norm

$$
\|f\|_{\mathcal{D}_{T}^{\gamma, \gamma_{0}, \mathfrak{N}}(M, \mathcal{G})}:=\sup _{t \leq T}\|f(t)\|_{\mathcal{D}^{t, \gamma, \mathfrak{N}}(M, \mathcal{G})}+\sup _{p \in M, s, t \leq T} \frac{\|f(t, p)-f(s, p)\|_{v}}{|t-s|^{\gamma_{0}}} .
$$

Remark 33 The modified norms with scaling parameter $\mathfrak{N}$ are necessary for the fixpoint argument, see Remark 39. As usual with Hölder-type spaces on compact domains, these spaces are complete metric spaces.

Remark 34 Note that we do not need transport in time, as opposed to the definition in [11, Definition 2.4].

The price we pay, is that Hölder regularity in time of the reconstruction of a solution is established in a roundabout way. Namely, by first verifying time regularity of the 0 component of the solution (Theorem 38) and then checking that reconstruction is given by projection on that component (Lemma 24).

In [11] it follows from the definition of a controlled distribution and their reconstruction theorem, Theorem 2.11 .

We now build the model for the structures $\mathcal{V}, \mathcal{W}$. As input we need realizations of $\boldsymbol{\Xi}$ and $\mathcal{I}[\boldsymbol{\Xi}] \boldsymbol{\Xi}$.

Definition 35 Assume for $T>0$ we are given $\xi \in C^{\alpha}(M)$ and a family of distributions $Z_{p}^{t} \in C^{\alpha}(M), t \in[0, T], p \in M$, satisfying

$$
\left|\left\langle Z_{p}^{t}, \varphi_{p}^{\lambda}\right\rangle\right| \lesssim \lambda^{2 \alpha+2}
$$




$$
Z_{q}^{t}(r)=Z_{p}^{t}(r)+\int_{0}^{t}\left\langle\mathrm{p}_{t-s}(p, \cdot)-\mathrm{p}_{t-s}(q, \cdot), \xi\right\rangle d s \xi(r),
$$

where the action of the heat kernel p on $\xi$ is well-defined by Theorem 37. Define

$$
\|\xi, Z\|_{\alpha, 2 \alpha+2, T}:=\|\xi\|_{C^{\alpha}(M)}+\sup _{t \leq T, q \in M, \lambda \in(0,1], \varphi \in \mathcal{B}_{T_{p} M}^{r, \delta}} \frac{\left|\left\langle Z_{q}^{t}, \varphi_{q}^{\lambda}\right\rangle\right|}{\lambda^{2 \alpha+2}}
$$

where $r:=-[\alpha]$ and $\delta$ is the radius of injectivity of $M$.

In our application to white-noise forcing, $\xi$ will be the white noise on $M$ and $Z$ will be constructed via Gaussian renormalization in Sect. 8 .

Now define the models for $\mathcal{V}$ and $\mathcal{W}$ as

$$
\begin{aligned}
\left(\Pi_{p}^{\mathcal{V}} \boldsymbol{\Xi}\right)(z) & =\xi(z) \\
\left(\Pi_{p}^{\mathcal{V}} \mathcal{I}[\boldsymbol{\Xi}] \boldsymbol{\Xi}\right)(z) & =Z_{p}^{t}(z) \\
\left(\Pi_{p}^{\mathcal{V}} \omega \mathbf{\Xi}\right)(z) & =\left(\Pi_{p}^{t} \omega\right)(z)\left(\Pi_{p}^{t} \boldsymbol{\Xi}\right)(z) \\
\left(\Pi_{p}^{\mathcal{W}} \mathbf{1}\right)(z) & =1 \\
\left(\Pi_{p}^{\mathcal{W}} \mathcal{I}[\boldsymbol{\Xi}]\right)(z) & =\int_{0}^{t}\left\langle\mathrm{p}_{t-r}(z, \cdot)-\mathrm{p}_{t-r}(p, \cdot), \xi\right\rangle d r \\
\left(\Pi_{p}^{\mathcal{W}} \omega\right)(z) & =\omega \exp _{p}^{-1}(z),
\end{aligned}
$$

with transports

$$
\begin{aligned}
\Gamma_{p \leftarrow q}^{t, \mathcal{V}} \boldsymbol{\Xi} & =\boldsymbol{\Xi} \\
\Gamma_{p \leftarrow q}^{t, \mathcal{V}} \mathcal{I}[\boldsymbol{\Xi}] \boldsymbol{\Xi} & =\mathcal{I}[\boldsymbol{\Xi}] \boldsymbol{\Xi}+\left[\int_{0}^{t}\left\langle\mathrm{p}_{t-r}(p, \cdot)-\mathrm{p}_{t-r}(q, \cdot), \xi\right\rangle d r\right] \boldsymbol{\Xi} \\
\Gamma_{p \leftarrow q}^{t, \mathcal{V}} \omega \boldsymbol{\Xi} & =\omega \exp _{q}^{-1}(p) \boldsymbol{\Xi}+d_{p}\left[\omega \exp _{q}^{-1}\right] \boldsymbol{\Xi} \\
\Gamma_{p \leftarrow q}^{t, \mathcal{W}} \mathbf{1} & =\mathbf{1} \\
\Gamma_{p \leftarrow q}^{t, \mathcal{W}} \mathcal{I}[\boldsymbol{\Xi}] & =\mathcal{I}[\boldsymbol{\Xi}]+\left[\int_{0}^{t}\left\langle\mathrm{p}_{t-r}(p, \cdot)-\mathrm{p}_{t-r}(q, \cdot), \xi\right\rangle d r\right] \mathbf{1} \\
\Gamma_{p \leftarrow q}^{t, \mathcal{W}} \omega & =\omega \exp _{q}^{-1}(p) \mathbf{1}+d_{p}\left[\omega \exp _{q}^{-1}\right] .
\end{aligned}
$$

Lemma 36 These are in fact models with $\delta_{M}=\delta$ the radius of injectivity of $M$ and the distances/norms of the model only depend on $\xi, Z$. Indeed for $\mathcal{G}=\mathcal{V}, \mathcal{W}, \gamma \in \mathbb{R}$

$$
\left\|\Pi^{t, \mathcal{G}}\right\|_{\beta ; \gamma ; M} \lesssim 1+\|\xi, Z\|_{\alpha, 2 \alpha+2, T}
$$

with $\beta=2$ for $\mathcal{G}=\mathcal{W}$ and $\beta=2+\alpha$ for $\mathcal{G}=\mathcal{V}$. 
Proof Let $\varphi \in \mathcal{B}_{T_{p} M}^{2, \delta_{M}}$. By Lemma 11

$$
\left|\left\langle\Pi_{p}^{t} \Xi, \varphi_{p}^{\lambda}\right\rangle\right|=\left|\left\langle\xi, \varphi_{p}^{\lambda}\right\rangle\right| \lesssim \lambda^{\alpha}\|\xi\|_{C^{\alpha}(M)} .
$$

By definition

$$
\left|\left\langle\Pi_{p}^{t} \mathcal{I}[\Xi] \Xi, \varphi_{p}^{\lambda}\right\rangle\right|=\left|\left\langle Z_{p}^{t}, \varphi_{p}^{\lambda}\right\rangle\right| \lesssim \lambda^{2 \alpha+2}|| \xi, Z \|_{\alpha, 2 \alpha+2, T} .
$$

Moreover

$$
\left|\left\langle\Pi_{p}^{t} \omega_{p} \Xi, \varphi_{p}^{\lambda}\right\rangle\right|=\left|\left\langle\xi, \omega_{p} \exp _{p}^{-1}(\cdot) \varphi_{p}^{\lambda}(\cdot)\right\rangle\right| \lesssim \lambda^{\alpha+1}|| \xi \|_{C^{\alpha}(M)}
$$

since $\omega_{p} \exp _{p}^{-1} \varphi_{p}^{\lambda}=\lambda \psi_{p}^{\lambda}$, with $\psi(\cdot)=\omega_{p}(\cdot) \phi(\cdot)$.

Regarding transport, both the transport of $\Xi$ and $\mathcal{I}[\Xi] \Xi$ are exact by definition and

$$
\begin{aligned}
&\left|\left\langle\Pi_{q}^{t, \mathcal{V}} \omega_{q} \Xi-\Pi_{p}^{t, \mathcal{V}} \Gamma_{p \leftarrow q} \omega_{q} \Xi, \varphi_{p}^{\lambda}\right\rangle\right|=\mid\left\langle\xi\left(\Pi_{q}^{t, \mathcal{W}} \omega_{q}-\Pi_{p}^{t, \mathcal{W}} \Gamma_{p \leftarrow q} \omega_{q}\right),\right. \\
&\left.\varphi_{p}^{\lambda}\right\rangle\left|\lesssim \lambda^{\alpha+2}\right| \mid \xi \|_{C^{\alpha}(M)},
\end{aligned}
$$

where we used Lemma 27 for the last step.

Finally

$$
\left\|\Gamma_{p \leftarrow q}^{t, \mathcal{V}} \mathcal{I}[\Xi] \Xi\right\|_{\alpha}=\left|\int_{0}^{t}\left\langle\mathrm{p}_{s}(p, \cdot)-\mathrm{p}_{s}(q, \cdot), \xi\right\rangle d s\right| \lesssim d(p, q)^{\alpha+2}
$$

by the Schauder estimate Theorem 37 , and

$$
\begin{aligned}
\left\|\Gamma_{p \leftarrow q}^{t, \mathcal{V}} \omega_{q} \Xi\right\|_{2 \alpha+2} & =0 \\
\left\|\Gamma_{p \leftarrow q}^{t, \mathcal{V}} \omega_{q} \Xi\right\|_{\alpha} & =\left\|\Gamma_{p \leftarrow q}^{t, \mathcal{W}} \omega_{q}\right\|_{0} \\
& \leq d(p, q)
\end{aligned}
$$

by Lemma 29. Hence

$$
\left\|\Pi^{t, \mathcal{V}} ; \Gamma^{t, \mathcal{V}}\right\|_{\beta, \gamma, M} \lesssim 1+\|\xi, Z\|_{\alpha, 2 \alpha+2, T}
$$

when $\beta:=2+\alpha$.

Analogously, one gets the bounds for $\mathcal{W}$ with $\beta=2$.

\section{Schauder estimates}

Let $p$ be the heat kernel on $M$. We start with a proof Schauder estimate for distributions, as a warm-up to the one for modelled distributions. 
Theorem 37 Let $T>0$, and $F \in L^{\infty}\left([0, T], C^{\alpha}(M)\right)$, for $\alpha \in(-2,-1)$. Then for $t \in[0, T]$

$$
\left|\int_{0}^{t}\left\langle p_{t-r}(p, \cdot), F(r)\right\rangle d r-\int_{0}^{t}\left\langle p_{t-r}(q, \cdot), F(r)\right\rangle d r\right| \lesssim \sup _{t \leq T}\|F(t)\|_{C^{\alpha}(M)} d(p, q)^{2+\alpha} .
$$

Proof As in the proof of the next theorem we shall focus on the singular part $\mathrm{p}^{N}$ in the decomposition $\mathrm{p}=\mathrm{p}^{N}+R^{N}$ using heat asymptotics, Theorem 43 , for arbitrary large $N$. Let us set $\|F\|=\sup _{t \leq T}\left\|F_{t}\right\|_{C^{\alpha}(M)}, d=d(p, q)$ and let $\gamma$ be a geodesic path from $p$ to $q$ of constant speed $d$. We single out the singularity of the heat kernel considering separately the cases $t \leq d^{2}$ and $t>d^{2}$ where we bound the integral over $\left(0, t-d^{2}\right)$ and $\left(t-d^{2}, t\right)$. Close to the singularity, using the first item of Lemma 44,

$$
\sup _{z \in M}\left|\int_{t-d^{2}}^{t}\left\langle\mathrm{p}_{t-r}^{N}(z, \cdot), F(r)\right\rangle d r\right| \lesssim\|F\| \int_{0}^{d^{2}} r^{\frac{\alpha}{2}} d r=\|F\| d^{\alpha+2}
$$

for $t>d^{2}$, whereas for $t \leq d^{2}$,

$$
\sup _{z \in M} \mid \int_{0}^{t}\left\langle\mathrm{p}_{t-r}^{N}(z, \cdot), F(r)|d r| \lesssim\|F\| t^{\alpha+2} \leq\|F\| d^{\alpha+2}\right.
$$

When $t>d^{2}$, we write on the other interval,

$$
\begin{aligned}
& \left|\int_{0}^{t-d^{2}}\left\langle\mathrm{p}_{t-r}^{N}(p, \cdot), F(r)\right\rangle d r-\int_{0}^{t-d^{2}}\left\langle\mathrm{p}_{t-r}^{N}(q, \cdot), F(r)\right\rangle d r\right| \\
& \quad=\int_{0}^{1} \int_{0}^{t-d^{2}}\left\langle\nabla_{\dot{\gamma}_{u}} \mathrm{p}_{t-r}^{N}(\bullet, \cdot), F(r)\right\rangle d r d u,
\end{aligned}
$$

where • stands for the variable that is being differentiated and $\cdot$ is the variable in the distribution's pairing. Then, according to the second item of Lemma 44, for any $u \in(0,1)$,

$$
\int_{0}^{t-d^{2}}\left\langle\nabla_{\dot{\gamma}_{u}} \mathrm{p}_{t-r}^{N}(\bullet, \cdot), F(r)\right\rangle d r \lesssim\|F\| \int_{d^{2}}^{t} d \sqrt{r}^{\alpha-1} d r \leq\|F\| d^{\alpha+2}
$$

The latter four inequalities yield the claim for the singular part of the heat kernel. Now, according to Theorem 43, for $N$ large enough,

$$
\sup _{u \in(0,1), r \in(0, t)}\left\langle\nabla_{\dot{\gamma}_{u}} R_{t-r}^{N}(\bullet, \cdot), F(r)\right\rangle \lesssim d\|F\| \leq d^{\alpha+2}\|F\| .
$$

This concludes the proof. 
We now prove an extension of this classical result to the space of modelled distributions. For ${ }^{4}$

$$
f(t, p)=f_{\alpha}(t, p) \boldsymbol{\Xi}+f_{2+2 \alpha}(t, p) \mathcal{I}[\boldsymbol{\Xi}] \boldsymbol{\Xi}+f_{1+\alpha}(t, p) \boldsymbol{\Xi},
$$

an element of $\mathcal{D}_{T}^{\gamma, \gamma_{0}}(\mathcal{V})$, define

$$
\left(\mathcal{K}_{t} f\right)(p):=h:=h_{0}(t, p) \mathbf{1}+h_{2+\alpha}(t, p) \mathcal{I}[\boldsymbol{\Xi}]+h_{1}(t, p),
$$

with

$$
\begin{aligned}
h_{0}(t, p) & =\int_{0}^{t}\left\langle\mathrm{p}_{t-s}(p, \cdot), \mathcal{R}_{s} f(s)\right\rangle d s \\
h_{2+\alpha}(t, p) & =f_{\alpha}(t, p) \\
h_{1}(t, p) & =\left.d\right|_{p}\left(z \mapsto \int_{0}^{t}\left\langle\mathrm{p}_{t-s}(z, \cdot), \mathcal{R}_{s} f(s)-f_{\alpha}(t, p) \Pi_{p}^{t} \mathbf{\Xi}\right\rangle d s\right)
\end{aligned}
$$

The well-definedness of these terms is part of the following theorem.

Theorem 38 (Schauder estimate) For $\alpha \in(-4 / 3,-1)$, with $\gamma \in(0,2 \alpha+8 / 3)$, set $\varepsilon:=(2 \alpha+8 / 3-\gamma) / 4$ and $\gamma_{0}=\alpha / 2+1-\varepsilon$. Let $T>0$ and $f \in \mathcal{D}_{T}^{\gamma, \gamma_{0}}(\mathcal{V})$. Then, for all $t \in[0, T],{ }^{5}$

$$
\mathcal{R}_{t} \mathcal{K} f=\int_{0}^{t}\left\langle p_{t-s}, \mathcal{R}_{s} f(s)\right\rangle d s .
$$

Moreover, $\mathcal{K} f \in \mathcal{D}^{\bar{\gamma}, \bar{\gamma}_{0}}(\mathcal{W})$, with $\bar{\gamma}=\gamma+4 / 3, \bar{\gamma}_{0}=\gamma_{0}$ and

$$
\|\mathcal{K} f\|_{\mathcal{D}_{T}^{\bar{\gamma}, \bar{\gamma}_{0}, \mathfrak{N}}(\mathcal{W})} \lesssim\|f\|_{\mathcal{D}_{T}^{\gamma, \gamma_{0}, \mathfrak{N}}(\mathcal{V})}\left(T^{\varepsilon}+T^{\varepsilon} \mathfrak{N}+\frac{1}{\mathfrak{N}}\right) .
$$

Remark 39 Here we can see why we introduced the modified norm $\|.\|_{\mathcal{D}_{T}^{\bar{\gamma}, \bar{\gamma}_{0}, \mathfrak{N}}(\mathcal{W})}$. Without it, i.e. with $\mathfrak{N} \equiv 1$, the factor on the right hand side cannot be made small, which is necessary for the fixpoint argument.

Remark 40 Contrary to classical Schauder estimates, we only get an "improvement of $4 / 3$ derivatives". In order to get an "improvement of 2 derivatives" one has to include quadratic polynomials in the regularity structure. This is also the reason why we have to choose $\gamma, \gamma_{0}$ in such a specific way.

Note that an improvement by $4 / 3$ will be enough to set up the fix-point argument.

To be specific, in order to get an "improvement of 2 derivatives" the complete list of symbols necessary is, ordered by homogeneity,

\footnotetext{
${ }^{4}$ Recall from the beginning of this section that $f_{\alpha}, f_{2+2 \alpha}$ are real-valued and $f_{1+\alpha}$ is a section of $T^{*} M$.

${ }^{5}$ Recall that $\mathcal{R}_{t}$ is the reconstruction operator of Theorem 23 associated to the model $\left(\Pi^{t}, \Gamma^{t}\right)$. 


$$
\begin{aligned}
& \Xi, \Xi \mathcal{I}[\Xi], \Xi X_{i}, 1, \Xi \mathcal{I}[\Xi \mathcal{I}[\Xi]], \Xi \mathcal{I}\left[\Xi X_{i}\right], \mathcal{I}[\Xi], \\
& \Xi X_{i} X_{j}, \Xi X_{1}, X_{i}, \mathcal{I}[\Xi \mathcal{I}[\Xi]], \mathcal{I}\left[\Xi X_{i}\right], X_{i} X_{j}, X_{1},
\end{aligned}
$$

where $i, j=2,3$ stand for the space-directions. ${ }^{6}$ These symbols would be the building blocks for the regularity structure on flat space. On a manifold the polynomials would represent the respective symmetric covariant tensor bundles, as laid out in Sect. 9. The Schauder estimate has to be shown on the level of each of theses symbols, and hence a treatment "by hand", as we do here, would be cumbersome.

Remark 41 The following proof based on the heat kernel (almost) being a scaled test function goes back, in the flat case, to [4]. A proof splitting up the heat kernel into a sum of smooth, compactly supported kernels (following the strategy of [9]) is also possible, but less convenient.

Proof of Theorem 38 Once the second statement is established, the first one follows from the definition of $h_{0}$ and the fact that reconstruction of modelled distributions taking values only in positive homogeneities is given by the projection onto homogeneity 0 , see Lemma 24.

Recall that $\delta_{M}=\delta$, the radius of injectivity. By Remark 19 we can, and will only consider points at distance less than $\delta / 4$.

Introduce the short notation

$$
\begin{aligned}
C_{f} & :=\|f\|_{\mathcal{D}_{T}^{\gamma, \gamma_{0}, \mathfrak{N}}}(M, \mathcal{V}) \\
C_{\Pi} & :=\sup _{t \leq T}\left\|\Pi^{t, \mathcal{V}}, \Gamma^{t, \mathcal{V}}\right\|_{\beta, M} .
\end{aligned}
$$

Note that $\|\xi\|_{C^{\alpha}(M)} \leq C_{\Pi}$.

We shall need the following facts. Since

$$
\left\|f(t, p)-\Gamma_{p \leftarrow q}^{t} f(t, q)\right\|_{\alpha} \lesssim \frac{C_{f}}{\mathfrak{N}} d(p, q)^{\gamma-\alpha},
$$

we have

$$
\begin{aligned}
& \left|f_{\alpha}(t, p)-f_{\alpha}(t, q)\right| \\
& \quad \lesssim|| f(t, p)-\left.\Gamma_{p \leftarrow q}^{t} f(t, q)\right|_{\alpha}+\left|f_{2 \alpha+2}(t, q) \int_{0}^{t}\left\langle\mathrm{p}_{t-s}(p, \cdot)-\mathrm{p}_{t-s}(q, \cdot), \xi\right\rangle d s\right| \\
& \quad+\left|f_{1+\alpha}(t, q) \exp _{q}^{-1}(p)\right| \\
& \quad \lesssim \frac{C_{f}}{\mathfrak{N}} d(p, q)^{\gamma-\alpha}+C_{f}\|\xi\|_{C^{\alpha}(M)} d(p, q)^{2+\alpha}+C_{f} d(p, q) \\
& \quad \lesssim\left(\frac{C_{f}}{\mathfrak{N}}+C_{f}+C_{f} C_{\Pi}\right) d(p, q)^{2+\alpha}
\end{aligned}
$$

where we used the classical Schauder estimate Theorem 37.

\footnotetext{
6 Assuming that one builds a regularity structure including space and time.
} 
Moreover for a function $\varphi$ satisfying the assumptions of Lemmas 17 and 25

$$
\begin{aligned}
\left|\left\langle\mathcal{R}_{t} f(t)-f_{\alpha}(t, p) \xi, \varphi \circ \exp _{p}^{-1}\right\rangle\right| \\
\leq\left|\left\langle\mathcal{R}_{t} f(t)-\Pi_{p}^{t} f(t), \varphi \circ \exp _{p}^{-1}\right\rangle\right|+\left|\left\langle\Pi_{p}^{t} f(t)-f_{\alpha}(t, p) \xi, \varphi \circ \exp _{p}^{-1}\right\rangle\right| \\
=\left|\left\langle\mathcal{R}_{t} f(t)-\Pi_{p}^{t} f(t), \varphi \circ \exp _{p}^{-1}\right\rangle\right|+\mid\left\langle f_{2 \alpha+2}(t, p) \Pi_{p}^{t}(\mathcal{I}[\Xi] \Xi)\right. \\
\left.\quad+\Pi_{p}^{t}\left(f_{\alpha+1}(t, p) \Xi\right), \varphi \circ \exp _{p}^{-1}\right\rangle \mid \\
\leq C_{f} C_{\Pi} \lambda^{\gamma}+C_{f} C_{\Pi} \lambda^{2 \alpha+2}+C_{f} C_{\Pi} \lambda^{\alpha+1} \\
\lesssim C_{f} C_{\Pi} \lambda^{2 \alpha+2}
\end{aligned}
$$

and similarly

$$
\begin{aligned}
\left|\left\langle\mathcal{R}_{t} f(t), \varphi \circ \exp _{p}^{-1}\right\rangle\right| & \leq\left|\left\langle\mathcal{R}_{t} f(t)-\Pi_{p}^{t} f(t), \varphi \circ \exp _{p}^{-1}\right\rangle\right|+\left|\left\langle\Pi_{p}^{t} f(t), \varphi \circ \exp _{p}^{-1}\right\rangle\right| \\
& \lesssim C_{f} C_{\Pi} \lambda^{\gamma}+C_{f} C_{\Pi}\left(\lambda^{\alpha}+\lambda^{2 \alpha+2}+\lambda^{\alpha+1}\right) \\
& \lesssim C_{f} C_{\Pi} \lambda^{\alpha}
\end{aligned}
$$

With these estimates at hand, we shall now control each term in the definition of the norm $\|\mathcal{K} f\|_{\mathcal{D}_{T}^{\bar{\gamma}, \bar{\gamma}_{0}, \mathfrak{N}}(\mathcal{W})}$, using the decomposition of the heat kernel $\mathrm{p}=\mathrm{p}^{N}+R^{N}$, from Theorem 43, for $N$ large enough, as we did above for the classical Schauder estimate.

\section{Space regularity}

\section{Homogeneity 0}

This term can be written as

$$
\begin{aligned}
\left(h(t, p)-\Gamma_{p \leftarrow q}^{t} h(t, q)\right)_{0} \\
\left.=h_{0}(t, p)-h_{0}(t, q)-h_{\alpha+2}(t, q)\left(\Gamma_{p \leftarrow q}^{t} \mathcal{I}[\boldsymbol{\Xi}]\right)_{0}-\left(\Gamma_{p \leftarrow q}^{t} h_{1}(t, q)\right)\right)_{0} \\
=\int_{0}^{t}\left\langle\mathrm{p}_{t-s}(p, \cdot), \mathcal{R}_{s} f(s)\right\rangle d s-\int_{0}^{t}\left\langle\mathrm{p}_{t-s}(q, \cdot), \mathcal{R}_{s} f(s)\right\rangle d s \\
\quad-f_{\alpha}(t, q) \int_{0}^{t}\left\langle\mathrm{p}_{t-r}(p, \cdot)-\mathrm{p}_{t-r}(q, \cdot), \xi\right\rangle d r \\
\quad-\left.d\right|_{q}\left(z \mapsto \int_{0}^{t}\left\langle\mathrm{p}_{t-s}(z, \cdot), \mathcal{R}_{s} f(s)-f_{\alpha}(t, q) \Pi_{q}^{t} \Xi\right\rangle d s\right) \exp _{q}^{-1}(p) \\
=\int_{0}^{t}\left\langle\mathrm{p}_{t-s}(p, \cdot)-\mathrm{p}_{t-s}(q, \cdot)-\left.d\right|_{q} \mathrm{p}_{t-s}(q, \cdot)\left(\exp _{q}^{-1}(p)\right), \mathcal{R}_{s} f(s)-f_{\alpha}(t, q) \xi\right\rangle d s .
\end{aligned}
$$

Regarding the contribution of the regular part $R^{N}$ of the heat kernel, we write

$$
\begin{gathered}
\int_{0}^{t}\left\langle R_{t-s}^{N}(p, \cdot)-R_{t-s}^{N}(q, \cdot)-\left.d\right|_{q} R_{t-s}^{N}(q, \cdot) \exp _{q}^{-1}(p), \mathcal{R}_{s} f(s)-f_{\alpha}(t, q) \xi\right\rangle d s \\
\quad=\frac{1}{2} \int_{0}^{t}\left\langle\left.\int_{0}^{1} \nabla^{2}\right|_{\gamma(\theta)} R^{N}(\bullet, \cdot)\left(\dot{\gamma}(\theta)^{\otimes 2}\right)(1-\theta)^{2} d \theta, \mathcal{R}_{s} f(s)-f_{\alpha}(t, q) \xi\right\rangle d s
\end{gathered}
$$


where $\nabla$ acts on the dummy variable $\bullet$ and convolution acts on $\cdot$ and $\gamma$ is the geodesic connection $q$ to $p$. Since

$$
\left\|\mathcal{R}_{s} f(s)-f_{\alpha}(t, q) \xi\right\|_{\alpha} \lesssim C_{f} C_{\Pi}
$$

this expression is well-defined for $N$ large enough and of order

$$
C_{f} C_{\Pi} \sup _{\theta \leq 1}|\dot{\gamma}(\theta)|^{2}=C_{f} C_{\Pi} d(p, q)^{2}
$$

We now treat the term involving $\mathrm{p}^{N}$. Denoting by $g(t, s)$ the integrand of the above integral, for $s \in\left[t-d(p, q)^{2}, t\right]$,

$$
\begin{aligned}
|g(t, s)| \leq & \left|\left\langle\mathrm{p}_{t-s}^{N}(p, \cdot), \mathcal{R}_{s} f(s)-f_{\alpha}(t, q) \xi\right\rangle\right|+||\left\langle\mathrm{p}_{t-s}^{N}(q, \cdot), \mathcal{R}_{s} f(s)-f_{\alpha}(t, q) \xi\right\rangle \mid \\
& +\left|\left\langle\left. d\right|_{q} \mathrm{p}_{t-s}^{N}(q, \cdot)\left(\exp _{q}^{-1}(p)\right), \mathcal{R}_{s} f(s)-f_{\alpha}(t, q) \xi\right\rangle\right| .
\end{aligned}
$$

The first term we bound as

$$
\begin{aligned}
\left|\left\langle\mathrm{p}_{t-s}^{N}(p, \cdot), \mathcal{R}_{s} f(s)-f_{\alpha}(t, q) \xi\right\rangle\right| \leq & \left|\left\langle\mathrm{p}_{t-s}^{N}(p, \cdot), \mathcal{R}_{s} f(s)-f_{\alpha}(s, p) \xi\right\rangle\right| \\
& +\left|\left\langle\mathrm{p}_{t-s}^{N}(p, \cdot),\left(f_{\alpha}(s, p)-f_{\alpha}(t, q)\right) \xi\right\rangle\right| \\
\lesssim & C_{f} C_{\Pi}|t-s|^{(2 \alpha+2) / 2} \\
& +C_{\Pi}|t-s|^{\alpha / 2}\left(\left(\frac{C_{f}}{\mathfrak{N}}+C_{f}+C_{f} C_{\Pi}\right) d(p, q)^{2+\alpha}\right. \\
& \left.+C_{f}|t-s|^{\gamma_{0}}\right),
\end{aligned}
$$

where we used (10) together with Lemma 44 (i), as well as the Hölder continuity of $f_{\alpha}$ in space (9) and in time.

The second we bound as

$$
\begin{aligned}
& \left|\left\langle\mathrm{p}_{t-s}^{N}(q, \cdot), \mathcal{R}_{s} f(s)-f_{\alpha}(t, q) \xi\right\rangle\right| \\
& \quad \leq\left|\left\langle\mathrm{p}_{t-s}^{N}(q, \cdot), \mathcal{R}_{s} f(s)-f_{\alpha}(s, q) \xi\right\rangle\right|+\left|\left\langle\mathrm{p}_{t-s}^{N}(q, \cdot),\left(f_{\alpha}(s, q)-f_{\alpha}(t, q)\right) \xi\right\rangle\right| \\
& \quad \lesssim C_{f} C_{\Pi}|t-s|^{(2 \alpha+2) / 2}+C_{\Pi} C_{f}|t-s|^{\alpha / 2}|t-s|^{\gamma_{0}},
\end{aligned}
$$

where we used (10) together with Lemma 44 (i) as well as the Hölder continuity of $f_{\alpha}$ in time.

The last one we bound as

$$
\begin{aligned}
& \left|\left\langle\left. d\right|_{q} \mathrm{p}_{t-s}^{N}(q, \cdot) \exp _{q}^{-1}(p), \mathcal{R}_{s} f(s)-f_{\alpha}(t, q) \xi\right\rangle\right| \\
& \quad \leq\left|\left\langle\left. d\right|_{q} \mathrm{p}_{t-s}^{N}(q, \cdot) \exp _{q}^{-1}(p), \mathcal{R}_{s} f(s)-f_{\alpha}(s, q) \xi\right\rangle\right|
\end{aligned}
$$




$$
\begin{aligned}
& +\left|\left\langle\left. d\right|_{q} \mathrm{p}_{t-s}^{N}(q, \cdot) \exp _{q}^{-1}(p),\left(f_{\alpha}(s, q)-f_{\alpha}(t, q)\right) \xi\right\rangle\right| \\
\lesssim & C_{f} C_{\Pi} d(p, q)|t-s|^{(2 \alpha+2) / 2-1 / 2}+C_{\Pi} d(p, q)|t-s|^{\alpha / 2-1 / 2} C_{f}|t-s|^{\gamma_{0}}
\end{aligned}
$$

where we used (10) together with Lemma 44 (ii) as well as the Hölder continuity of $f_{\alpha}$ in space (9) and in time.

Hence

$$
\begin{aligned}
|g(t, s)| \lesssim & \left(|t-s|^{1 / 2}+d(p, q)\right) \\
& \times\left(C_{f} C_{\Pi}|t-s|^{(2 \alpha+2) / 2-1 / 2}\right. \\
& +C_{\Pi}\left(\frac{C_{f}}{\mathfrak{N}}+C_{f}+C_{f} C_{\Pi}\right) d(p, q)^{2+\alpha}|t-s|^{\alpha / 2-1 / 2} \\
& \left.+C_{\Pi} C_{f}|t-s|^{\alpha / 2-1 / 2}|t-s|^{\gamma_{0}}\right)
\end{aligned}
$$

and then by Lemma 42

$$
\begin{aligned}
\int_{t-d(p, q)^{2}}^{t}|g(t, s)| d s \lesssim & T^{\varepsilon}\left(C_{f} C_{\Pi} d(p, q)^{2 \alpha+4-2 \varepsilon}\right. \\
& +C_{\Pi}\left(\frac{C_{f}}{\mathfrak{N}}+C_{f}+C_{f} C_{\Pi}\right) d(p, q)^{2 \alpha+4-2 \varepsilon} \\
& \left.+C_{\Pi} C_{f} d(p, q)^{\alpha+2+2 \gamma_{0}-2 \varepsilon}\right)
\end{aligned}
$$

if

$(2 \alpha+2) / 2, \alpha / 2+\gamma_{0}, \alpha / 2,(\alpha+2) / 2-1 / 2, \alpha / 2-1 / 2+\gamma_{0}>-1+\varepsilon$.

Then the following are upper bounds to $\bar{\gamma}$

$$
2 \alpha+4-2 \varepsilon, \alpha+2 \gamma_{0}+2-2 \varepsilon \text {. }
$$

Both are satisfied under our assumptions.

Now consider $s \in\left[0, t-d(p, q)^{2}\right]$. By Theorem 62 we have

$$
\begin{aligned}
& \mathrm{p}_{t-s}^{N}(p, \cdot)-\mathrm{p}_{t-s}^{N}(q, \cdot)-\left.d\right|_{q} \mathrm{p}_{t-s}^{N}(q, \cdot) \exp _{q}^{-1}(p) \\
& \quad=\int_{0}^{1} \nabla^{2} \mathrm{p}_{t-s}^{N}(\gamma(r), \cdot)(\dot{\gamma}(r) \otimes \dot{\gamma}(r))(1-r) d r,
\end{aligned}
$$

where $\gamma(r):=\exp _{q}(r v), v:=\exp _{q}^{-1}(p)$, for any $r \in[0,1]$, and $\nabla^{2}$ is acting on the first variable of $\mathrm{p}^{N}$. Now

$$
g(t, s)=\left\langle\mathrm{p}_{t-s}^{N}(p, \cdot)-\mathrm{p}_{t-s}^{N}(q, \cdot)-\left.d\right|_{q} \mathrm{p}_{t-s}^{N}(q, \cdot) \exp _{q}^{-1}(p), \mathcal{R}_{s} f(s)-f_{\alpha}(t, q) \xi\right\rangle
$$




$$
\begin{aligned}
= & \left\langle\mathbf{p}_{t-s}^{N}(p, \cdot)-\mathbf{p}_{t-s}^{N}(q, \cdot)-\left.d\right|_{q} \mathbf{p}_{t-s}^{N}(q, \cdot) \exp _{q}^{-1}(p), \mathcal{R}_{s} f(s)-f_{\alpha}(s, q) \xi\right\rangle \\
& +\left\langle\mathbf{p}_{t-s}^{N}(p, \cdot)-\mathbf{p}_{t-s}^{N}(q, \cdot)-\left.d\right|_{q} \mathbf{p}_{t-s}^{N}(q, \cdot) \exp _{q}^{-1}(p),\left(f_{\alpha}(s, q)-f_{\alpha}(t, q)\right) \xi\right\rangle
\end{aligned}
$$

The first term we bound as

$$
\begin{aligned}
& \left|\left\langle\mathrm{p}_{t-s}^{N}(p, \cdot)-\mathrm{p}_{t-s}^{N}(q, \cdot)-\left.d\right|_{q} \mathrm{p}_{t-s}^{N}(q, \cdot) \exp _{q}^{-1}(p), \mathcal{R}_{s} f(s)-f_{\alpha}(s, q) \xi\right\rangle\right| \\
& \quad=\mid \int_{0}^{1}\left\langle\left(\nabla^{2} \mathrm{p}_{t-s}(\gamma(r), \cdot)(\dot{\gamma}(r) \otimes \dot{\gamma}(r)), \mathcal{R}_{s} f(s)-f_{\alpha}(s, q) \xi\right\rangle(1-r) d r\right| \\
& \quad \lesssim \int_{0}^{1}|v|^{2} C_{f} C_{\Pi}|t-s|^{(2+2 \alpha) / 2-1}(1-r) d r \\
& \quad \lesssim|v|^{2} C_{f} C_{\Pi}|t-s|^{(2+2 \alpha) / 2-1} \\
& \quad=d(p, q)^{2} C_{f} C_{\Pi}|t-s|^{(2+2 \alpha) / 2-1},
\end{aligned}
$$

where we used (10) together with Lemma $44 .^{7}$

The second term we bound as

$$
\begin{aligned}
& \left|\left\langle\mathrm{p}_{t-s}^{N}(p, \cdot)-\mathrm{p}_{t-s}^{N}(q, \cdot)-\left.d\right|_{q} \mathrm{p}_{t-s}^{N}(q, \cdot) \exp _{q}^{-1}(p),\left(f_{\alpha}(s, q)-f_{\alpha}(t, q)\right) \xi\right\rangle\right| \\
& \quad=\mid \int_{0}^{1}\left\langle\left(\nabla d \mathrm{p}_{t-s}(\gamma(r), \cdot)(\dot{\gamma}(r) \otimes \dot{\gamma}(r)),\left(f_{\alpha}(s, q)-f_{\alpha}(t, q)\right) \xi\right\rangle d r\right| \\
& \quad \lesssim d(p, q)^{2} C_{\Pi} C_{f}|t-s|^{\alpha / 2-1}|t-s|^{\gamma_{0}},
\end{aligned}
$$

where we used Lemma 44 and the Hölder continuity of $f_{\alpha}$ in time.

Hence by Lemma 42

$$
\int_{0}^{t-d(p, q)^{2}} g(t, s) d s \lesssim T^{\varepsilon}\left(C_{f} C_{\Pi} d(p, q)^{4+2 \alpha-2 \varepsilon}+C_{\Pi} C_{f} d(p, q)^{\alpha+2+2 \gamma_{0}-2 \varepsilon}\right)
$$

if

$$
(2 \alpha+2) / 2-1, \alpha / 2+\gamma_{0}<-1+\varepsilon .
$$

Then the following are upper bounds to $\bar{\gamma}$

$$
2 \alpha+4-2 \varepsilon, 2+\alpha+2 \gamma_{0}-2 \varepsilon .
$$

7 In coordinates,

$$
\nabla^{2} \mathrm{p}_{t-s}^{n}(\gamma(r), \cdot)=\left(\partial_{i j} \mathrm{p}_{t-s}^{n}(\gamma(r), \cdot)-\sum_{k} \Gamma_{i j}^{k} \partial_{k} \mathrm{p}_{t-s}^{n}(\gamma(r), \cdot)\right) d x^{i} \otimes d x^{j}
$$

where $\Gamma$ are the Christoffel symbols. This gives the quadratic factor in $|\dot{\gamma}(r)|=d(p, q)$. The blowup in $t-s$ follows from an application of Lemma 44 (i), (ii) to the components here. 
Both are satisfied under our assumptions. Hence

$$
\mathfrak{N}\left\|f(t, p)-\Gamma_{p \leftarrow q}^{t} f(t, q)\right\|_{\alpha} \lesssim C_{f}\left(T^{\varepsilon}+T^{\varepsilon} \mathfrak{N}\right) d(p, q)^{\bar{\gamma}} .
$$

\section{Homogeneity $\alpha+2$}

$$
\begin{aligned}
\left\|h(t, p)-\Gamma_{p \leftarrow q}^{t} h(t, q)\right\|_{\alpha+2} & =\left|h_{\alpha+2}(t, p)-h_{\alpha+2}(t, q)\right| \\
& =\left|f_{\alpha}(t, p)-f_{\alpha}(t, q)\right| \\
& \lesssim \frac{1}{\mathfrak{N}}\|f\|_{\mathcal{D}_{T}^{\gamma, \mathfrak{N}}(\mathcal{V})} d(p, q)^{\gamma-\alpha} \\
& =\frac{1}{\mathfrak{N}}\|f\|_{\mathcal{D}_{T}^{\gamma, \mathfrak{N}}(\mathcal{V})} d(p, q)^{(\gamma+2)-(\alpha+2),}
\end{aligned}
$$

so we need

$$
\bar{\gamma} \leq \gamma+2
$$

which is satisfied under our assumptions.

\section{Homogeneity 1}

We only treat here the terms involving $\mathrm{p}^{N}$ within

$$
\begin{gathered}
\left(h(t, p)-\Gamma_{p \leftarrow q}^{t} h(t, q)\right)_{1}=\int_{0}^{t}\left\langle d \mathrm{p}_{t-s}(p, \cdot), \mathcal{R}_{s} f(s)-f_{\alpha}(t, p) \Pi_{p}^{t} \Xi\right\rangle d s \\
-\left.d\right|_{p}\left[z \mapsto \int_{0}^{t}\left\langle d \mathrm{p}_{t-s}(q, \cdot), \mathcal{R}_{s} f(s)-f_{\alpha}(t, q) \Pi_{q}^{t} \Xi\right\rangle d s \exp _{q}^{-1}(z)\right] .
\end{gathered}
$$

We write it as $\int_{0}^{t} g(t, s) d s$, with

$$
\begin{aligned}
g(t, s)= & \left\langle d \mathrm{p}_{t-s}^{N}(p, \cdot), \mathcal{R}_{s} f(s)-f_{\alpha}(t, p) \Pi_{p}^{t} \Xi\right\rangle-\left.d\right|_{p} \\
& {\left.\left[z \mapsto\left\langle d \mathrm{p}_{t-s}^{N}(q, \cdot), \mathcal{R}_{s} f(s)-f_{\alpha}(t, q) \Pi_{q}^{t} \boldsymbol{\Xi}\right\rangle\right)\right] . }
\end{aligned}
$$

It is enough to bound this expression acting on $X_{p} \in T_{p} M$. Write

$$
\zeta_{p}^{s}:=\mathcal{R}_{s} f(s)-f_{\alpha}(s, p) \Pi_{p}^{s} \Xi
$$

For $s \in\left[t-d(p, q)^{2}, t\right]$ we bound (• denotes the dummy variable on which $X_{p}$ is acting, $\cdot$ denotes the dummy variable in the distribution-pairing)

$$
\begin{aligned}
& \left|\left\langle\left. d\right|_{p} \mathbf{p}_{t-s}^{N}(\bullet, \cdot)\left(X_{p}\right), \mathcal{R}_{s} f(s)-f_{\alpha}(t, p) \Pi_{p}^{s} \boldsymbol{\Xi}\right\rangle\right| \\
& \quad \leq\left|\left\langle\left. d\right|_{p} \mathbf{p}_{t-s}^{N}(\bullet, \cdot)\left(X_{p}\right), \zeta_{q}^{s}\right\rangle\right|+\left|\left\langle\left. d\right|_{p} \mathbf{p}_{t-s}^{N}(\bullet, \cdot)\left(X_{p}\right),\left(f_{\alpha}(s, q)-f_{\alpha}(t, q)\right) \xi\right\rangle\right| \\
& \quad=\left|\left\langle X_{p}\left(\mathbf{p}_{t-s}^{N}(\bullet, \cdot)\right), \zeta_{p}^{s}\right\rangle\right|+\left|\left\langle X_{p}\left(\mathbf{p}_{t-s}^{N}(\bullet, \cdot)\right),\left(f_{\alpha}(s, q)-f_{\alpha}(t, q)\right) \xi\right\rangle\right|
\end{aligned}
$$




$$
\lesssim C_{f} C_{\Pi}|t-s|^{(2+2 \alpha) / 2-1 / 2}+C_{f} C_{\Pi}|t-s|^{\alpha / 2-1 / 2}|t-s|^{\gamma_{0}},
$$

where we used (10) together with Lemma 44 (ii), as well as the Hölder continuity of $f_{\alpha}$ in time.

Now

$$
\begin{aligned}
\left|\left\langle\left. d\right|_{p}\left[z \mapsto d \mathrm{p}_{t-s}^{N}(q, \cdot) \exp _{q}^{-1}(z)\right]\left(X_{p}\right), \mathcal{R}_{s} f(s)-f_{\alpha}(t, p) \Pi_{p}^{s} \Xi\right\rangle\right| \\
\leq\left|\left\langle\left. d\right|_{p}\left[z \mapsto d \mathrm{p}_{t-s}^{N}(q, \cdot) \exp _{q}^{-1}(z)\right]\left(X_{p}\right), \zeta_{q}^{s}\right\rangle\right| \\
\quad+\left|\left\langle\left. d\right|_{p}\left[z \mapsto d \mathrm{p}_{t-s}^{N}(q, \cdot) \exp _{q}^{-1}(z)\right]\left(X_{p}\right),\left(f_{\alpha}(s, q)-f_{\alpha}(t, q)\right) \xi\right\rangle\right| \\
=\left|\left\langle\left.\left. d\right|_{q} \mathrm{p}_{t-s}^{N}(q, \cdot) d\right|_{p} \exp _{q}^{-1}(z)\left(X_{p}\right), \zeta_{q}^{s}\right\rangle\right| \\
\quad+\left|\left\langle\left.\left. d\right|_{q} \mathrm{p}_{t-s}^{N}(q, \cdot) d\right|_{p} \exp _{q}^{-1}(z)\left(X_{p}\right),\left(f_{\alpha}(s, q)-f_{\alpha}(t, q)\right) \xi\right\rangle\right| \\
\lesssim C_{f} C_{\Pi}|t-s|^{(2+2 \alpha) / 2-1 / 2}+C_{f} C_{\Pi}|t-s|^{\alpha / 2-1 / 2}|t-s|^{\gamma_{0}},
\end{aligned}
$$

where we used (10) together with Lemma 44 (ii) with $Y_{p}:=\left.d\right|_{p} \exp _{q}^{-1}(z)\left(X_{p}\right)$, as well as the Hölder continuity of $f_{\alpha}$ in time.

Hence by Lemma 42

$$
\int_{t-d(p, q)^{2}}^{t}|g(t, s)| d s \lesssim T^{\varepsilon}\left(C_{f} C_{\Pi} d(p, q)^{3+2 \alpha-2 \varepsilon}+C_{f} C_{\Pi} d(p, q)^{\alpha+1+2 \gamma_{0}-2 \varepsilon}\right)
$$

if

$$
(1+2 \alpha) / 2, \alpha / 2-1 / 2+\gamma_{0}>-1+\varepsilon .
$$

Then the following are upper bounds to $\bar{\gamma}-1$

$$
3+2 \alpha-2 \varepsilon, \alpha+1+2 \gamma_{0}-2 \varepsilon \text {. }
$$

Both are satisfied under our assumptions.

Consider now $s \in\left[0, t-d(p, q)^{2}\right]$. Again it is enough to bound the term acting on some $X_{p} \in T_{p} M$. For notational simplicity let $v(z):=\left.\left.d\right|_{z} \mathrm{p}_{t-s}^{N}(z, \cdot) d\right|_{p} \exp _{z}^{-1}\left\langle X_{p}\right\rangle$ and $\zeta_{p}^{s}:=\mathcal{R}_{s} f(s)-f_{\alpha}(s, p) \xi$. We then write the term to bound as

$$
\begin{aligned}
\left\langle d \mathbf{p}_{t-s}^{N}(p, \cdot), \mathcal{R}_{s} f(s)-f_{\alpha}(t, p) \Pi_{p}^{t} \boldsymbol{\Xi}\right\rangle\left\langle X_{p}\right\rangle & \\
& -\left\langle\left. d\right|_{p}\left[z \mapsto d \mathrm{p}_{t-s}^{N}(q, \cdot) \exp _{q}^{-1}(z)\right], \mathcal{R}_{s} f(s)-f_{\alpha}(t, q) \Pi_{q}^{t} \boldsymbol{\Xi}\right\rangle\left\langle X_{p}\right\rangle \\
= & \left\langle v(p), \mathcal{R}_{s} f(s)-f_{\alpha}(t, p) \Pi_{p}^{t} \mathbf{\Xi}\right\rangle-\left\langle v(q), \mathcal{R}_{s} f(s)-f_{\alpha}(t, q) \Pi_{q}^{t} \mathbf{\Xi}\right\rangle \\
= & \left\langle v(p)-v(q), \zeta_{p}^{s}\right\rangle+\left\langle v(p),\left(f_{\alpha}(s, p)-f_{\alpha}(t, p)\right) \xi\right\rangle \\
& -\left\langle v(q),\left(f_{\alpha}(s, p)-f_{\alpha}(t, q)\right) \xi\right\rangle
\end{aligned}
$$




$$
\begin{aligned}
= & \left\langle v(p)-v(q), \zeta_{p}^{s}\right\rangle+\left\langle v(p)-v(q),\left(f_{\alpha}(s, p)-f_{\alpha}(t, p)\right) \xi\right\rangle \\
& +\left\langle v(q),\left(f_{\alpha}(t, q)-f_{\alpha}(t, p)\right) \xi\right\rangle .
\end{aligned}
$$

Now with $\gamma(t):=\exp _{q}(t v), v:=\exp _{q}^{-1}(p)$,

$$
\begin{aligned}
\left|\left\langle v(p)-v(q), \zeta_{p}^{s}\right\rangle\right| & =\left|\int_{0}^{1}\left\langle\left. d\right|_{\gamma(r)} v\langle\dot{\gamma}(r)\rangle, \zeta_{p}^{s}\right\rangle d r\right| \\
& \lesssim d(p, q) C_{f} C_{\Pi}|t-s|^{(2+2 \alpha) / 2-1}
\end{aligned}
$$

where we used (10) together with Lemma 44 (iii).

Similarly

$$
\begin{aligned}
& \left|\left\langle v(p)-v(q),\left(f_{\alpha}(s, p)-f_{\alpha}(t, p)\right) \xi\right\rangle\right| \\
& \quad=\left|\int_{0}^{1}\left\langle\left. d\right|_{\gamma(r)} v\langle\dot{\gamma}(r)\rangle,\left(f_{\alpha}(s, p)-f_{\alpha}(t, p)\right) \xi\right\rangle d r\right| \\
& \quad \lesssim d(p, q) C_{f} C_{\Pi}|t-s|^{\gamma_{0}}|t-s|^{\alpha / 2-1},
\end{aligned}
$$

where we used Lemma 44 (iii) and the Hölder continuity of $f_{\alpha}$ in time.

Finally

$$
\left|\left\langle v(q),\left(f_{\alpha}(t, q)-f_{\alpha}(t, p)\right) \xi\right\rangle\right| \lesssim\left(\frac{C_{f}}{\mathfrak{N}}+C_{f}+C_{f} C_{\Pi}\right) C_{\Pi} d(p, q)^{2+\alpha}|t-s|^{\alpha / 2-1 / 2}
$$

where we used Lemma 44 (ii) and the Hölder continuity of $f_{\alpha}$ in space (9).

Hence by Lemma 42

$$
\begin{aligned}
& \int_{0}^{t-d(p, q)^{2}}|g(t, s)| d s \lesssim T^{\varepsilon}\left(C_{f} C_{\Pi} d(p, q)^{3+2 \alpha-2 \varepsilon}\right. \\
& \left.+C_{f} C_{\Pi} d(p, q)^{\gamma_{0}+\alpha+1-2 \varepsilon}+\left(\frac{C_{f}}{\mathfrak{N}}+C_{f}+C_{f} C_{\Pi}\right) C_{\Pi} d(p, q)^{3+2 \alpha-2 \varepsilon}\right)
\end{aligned}
$$

if

$$
(2+2 \alpha-2) / 2, \alpha / 2-1 / 2, \alpha / 2-1+\gamma_{0}<-1+\varepsilon .
$$

Then the following are upper bounds for $\bar{\gamma}-1$

$$
2 \alpha+3-2 \varepsilon, 1+\alpha+\gamma_{0}-2 \varepsilon \text {. }
$$

Both are satisfied under our assumptions.

Then

$$
\left\|f(t, p)-\Gamma_{p \leftarrow q}^{t} f(t, q)\right\|_{1} \lesssim C_{f}\left(T^{\varepsilon}+T^{\varepsilon} \mathfrak{N}\right) d(p, q)^{\bar{\gamma}-1}
$$




\section{Time regularity}

Our definition requires only to bound the time increment in homogeneity 0 :

$$
\begin{aligned}
& h_{0}(t, p)-h_{0}(s, p) \\
& \quad=\int_{0}^{t}\left\langle\mathrm{p}_{t-r}(p, \cdot), \mathcal{R}_{r} f(r)\right\rangle d r-\int_{0}^{s}\left\langle\mathrm{p}_{s-r}(p, \cdot), \mathcal{R}_{r} f(r)\right\rangle d r \\
& \quad=\int_{s}^{t}\left\langle\mathrm{p}_{t-r}(p, \cdot), \mathcal{R}_{r} f(r)\right\rangle d r+\int_{0}^{s}\left\langle\mathrm{p}_{t-r}(p, \cdot)-\mathrm{p}_{s-r}(p, \cdot), \mathcal{R}_{r} f(r)\right\rangle d r .
\end{aligned}
$$

Let us consider first the regular part of the heat kernel. According to Theorem 43, for $N \geq 4$,

$$
K=\sup _{0<t \leq T, m \in\{0,1\},|\ell| \leq 2} t^{-1}\left\|\partial_{t}^{m} R_{t}^{N}\right\|_{C^{\ell}(M \times M)}<\infty .
$$

Together with the bound $\sup _{0 \leq r \leq T}\left\|\mathcal{R}_{r} f(r)\right\|_{C^{\alpha}}<\infty$, using a partition of unity, since $\alpha>-2$, it yields

$$
\left.\sup _{m \in\{0,1\}, 0<r<t \leq T}(t-r)^{-1}||\left\langle\partial_{t}^{m} R_{t-r}^{N}(p, \cdot), \mathcal{R}_{r} f(r)\right\rangle \mid\right\}<\infty .
$$

Up to a multiplicative constant, we can now bound the contribution of $R^{N}$ by

$$
\int_{s}^{t}(t-r) d r+\int_{0}^{s} \int_{s-r}^{t-r} \theta d \theta d r \lesssim t-s \lesssim T^{\varepsilon}(t-s)^{\bar{\gamma}_{0}}
$$

We now treat the term involving $\mathrm{p}^{N}$. Using (11) and Lemma 44 (i)

$$
\begin{aligned}
\int_{s}^{t}\left|\left\langle\mathrm{p}_{t-r}^{N}(p, \cdot), \mathcal{R}_{r} f(r)\right\rangle\right| d r & \lesssim C_{f} C_{\Pi} \int_{s}^{t}(t-r)^{\alpha / 2} d r \\
& =C_{f} C_{\Pi} \int_{s}^{t}(t-r)^{\varepsilon}(t-r)^{\alpha / 2-\varepsilon} d r \\
& \lesssim T^{\varepsilon} C_{f} C_{\Pi}|t-s|^{(\alpha+2) / 2-\varepsilon}
\end{aligned}
$$

Further, again using (11) and Lemma 44 (i)

$$
\begin{aligned}
& \int_{0}^{s}\left|\left\langle\mathrm{p}_{t-r}^{N}(p, \cdot)-\mathrm{p}_{s-r}^{N}(p, \cdot), \mathcal{R}_{r} f(r)\right\rangle\right| d r=\int_{0}^{s}\left|\int_{s-r}^{t-r}\left\langle\partial_{t} \mathrm{p}_{\theta}^{N}(p, \cdot), \mathcal{R}_{r} f(r)\right\rangle d \theta\right| d r \\
& \quad \lesssim C_{f} C_{\Pi} \int_{0}^{s} \int_{s-r}^{t-r} \theta^{\alpha / 2-1} d \theta d r \\
& \quad=C_{f} C_{\Pi} \frac{1}{(\alpha / 2)} \int_{0}^{s}\left[(t-r)^{\alpha / 2}-(s-r)^{\alpha / 2}\right] d r \\
& \quad \leq T^{\varepsilon} C_{f} C_{\Pi}\left|\frac{1}{(\alpha / 2)} \int_{0}^{s}\left[(t-r)^{\alpha / 2-\varepsilon}-(s-r)^{\alpha / 2-\varepsilon}\right] d r\right|
\end{aligned}
$$




$$
\begin{aligned}
= & T^{\varepsilon} C_{f} C_{\Pi} \mid-\frac{1}{(\alpha / 2)} \frac{1}{(\alpha / 2)+1-\varepsilon} \\
& \times\left[|t-s|^{\alpha / 2+1-\varepsilon}-t^{\alpha / 2+1-\varepsilon}+s^{\alpha / 2+1-\varepsilon}\right] \mid \\
\lesssim & T^{\varepsilon} C_{f} C_{\Pi}|t-s|^{\alpha / 2+1-\varepsilon},
\end{aligned}
$$

if

$$
\alpha / 2-\varepsilon>-1
$$

We then need

$$
\bar{\gamma}_{0}-\varepsilon \leq \alpha / 2+1-\varepsilon \text {. }
$$

Both are satisfied under our assumptions.

Then

$$
\left|h_{0}(t, p)-h_{0}(s, p)\right| \lesssim T^{\varepsilon} C_{f}|t-s|^{\bar{\gamma}_{0}}
$$

We used the following results.

Lemma 42 Let $\rho_{1}, \rho_{2} \in \mathbb{R}, g: \mathbb{R}^{2} \rightarrow[0, \infty)$ and assume for $A \leq t \leq T$,

$$
\begin{aligned}
& g(t, s) \leq C_{1}|t-s|^{\rho_{1}}, s \in[t-A, t] \\
& g(t, s) \leq C_{2}|t-s|^{\rho_{2}}, s \in[0, t-A] .
\end{aligned}
$$

Let $\rho_{1}>-1$ and $\varepsilon \geq 0$ such that $\rho_{2}-\varepsilon<-1$. Then

$$
\begin{aligned}
& \int_{t-A}^{t} g(t, s) d s \lesssim C_{1} A^{\rho_{1}+1} \lesssim C_{1} T^{\varepsilon} A^{\rho_{1}+1-\varepsilon} \\
& \int_{0}^{t-A} g(t, s) d s \lesssim C_{2} T^{\varepsilon} A^{\rho_{2}+1-\varepsilon} .
\end{aligned}
$$

Proof Indeed

$$
\int_{t-A}^{t} g(t, s) d s \leq C_{1} \int_{t-A}^{t}|t-s|^{\rho_{1}} d s \lesssim C_{1} A^{\rho_{1}+1}
$$

and

$$
\int_{0}^{t-A} g(t, s) d s \leq \int_{0}^{t-A}|t-s|^{\rho_{2}} d s \leq T^{\varepsilon} \int_{0}^{t-A}|t-s|^{\rho_{2}-\varepsilon} d s \lesssim T^{\varepsilon} A^{\rho_{2}+1-\varepsilon} .
$$


The following result on heat kernel asymptotics is classical and its proof can be found for example in [1, Theorem 2.30]; see also [15, Section 3.2]. In these references the norm $\|\cdot\|_{C^{\ell}(M \times M)}$ is defined via a partition of unity as in Definition 10 . There is a slight difference to our notation. In the cited references, $C^{1}$ for example means "continuously differentiable", while in our notation it only means "Lipschitz continuous". But it is enough to know that our norm is dominated by the norm in the references.

Theorem 43 Let $M$ be ad-dimensional, closed Riemannian manifold and $p$ be the heat kernel on $M$. Then there exist smooth functions $\left(\Phi_{i}(p, q)\right)_{i \geq 0}$ with $\Phi_{i}(p, q)=0$, for $d(p, q) \geq \delta / 4$, such that if we define for $N \geq 1$

$$
p^{N}(t, p, q):=t^{-d / 2} \exp \left(-\frac{d(p, q)^{2}}{4 t}\right) \sum_{i=0}^{N} t^{i} \Phi_{i}(p, q)
$$

we have

$$
\left\|\partial_{t}^{k}\left(p_{t}-p_{t}^{N}\right)\right\|_{C^{\ell}(M \times M)} \lesssim t^{N-d / 2-\ell / 2-k}
$$

Moreover for all $p \in M$

$$
\Phi_{0}(p, p)=1
$$

Lemma 44 Let

$$
p_{t}^{N}(p, q)=t^{-d / 2} \exp \left(-\frac{d(p, q)^{2}}{4 t}\right) \sum_{i=0}^{N} t^{i} \Phi_{i}(p, q)
$$

$\psi, \Phi_{i}$ smooth and with $\Phi_{i}(p, q)=0$, for $d(p, q) \geq \delta / 4$.

Let $p \in M$ and define for $z$ in the range of $\exp _{p}^{-1}, Y_{p} \in T_{p} M$ a tangent vector and $Z \in \Gamma(T M)$ a vector field

$$
\begin{aligned}
\varphi_{t}(z) & :=p_{t}^{N}\left(p, \exp _{p}(z)\right) \\
\varphi_{t}^{Y_{p}}(z) & :=Y_{p} p_{t}^{N}\left(\bullet, \exp _{p}(z)\right) \quad\left(Y_{p} \text { acting on } \bullet\right) \\
\varphi_{t}^{Y_{p}, Z}(z) & :=\left.Y_{p}\left[* \mapsto Z_{*} p_{t}^{N}(\bullet, \cdot)\right]\right|_{\cdot=\exp _{p}(z) \quad\left(Y_{p} \text { acting on } *, Z_{*} \text { acting on } \bullet\right) .}
\end{aligned}
$$

(Note that because of the small support of $p^{N}$, these are globally well-defined smooth functions by continuation with zero outside of the range of $\exp _{p}^{-1}$.)

Then for any multiindex $k$, any $n \geq 0$ and $\ell=0,1$.

$\begin{aligned} \text { (i) }\left|\partial_{t}^{\ell} D^{k} \varphi_{t}(z)\right| & \lesssim_{\ell, n, k}(\sqrt{t})^{-d-k-2 \ell} \frac{1}{1+(|z| / \sqrt{t})^{n}}, \\ \text { (ii) }\left|D^{k} \varphi_{t}^{Y}(z)\right| & \lesssim_{n, k}\left|Y_{p}\right|(\sqrt{t})^{-d-1-k} \frac{1}{1+(|z| / \sqrt{t})^{n}}, \\ \text { (iii) }\left|D^{k} \varphi_{t}^{Y_{p}, Z}(z)\right| & \lesssim_{Z, n, k}\left|Y_{p}\right|(\sqrt{t})^{-d-2-k} \frac{1}{1+(|z| / \sqrt{t})^{n}} .\end{aligned}$ 
Proof The summands of $\mathrm{p}^{N}$ are of the same form, apart from the factors $t^{i}, i=$ $0, \ldots, N$. Since for $i \geq 1$ they improve the singularity at $t=0$, it is enough to treat $N=0$.

Then

$$
\varphi_{t}(z)=t^{-d / 2} \exp \left(-\frac{|z|^{2}}{4 t}\right) \Phi\left(p, \exp _{p}(z)\right)
$$

Since $z \mapsto \Phi\left(p, \exp _{p}(z)\right)$ is smooth, uniformly in $p$, with support in $B_{T_{p} M}\left(0_{p}, \delta / 4\right)$ and the factor $1 / 4$ in the exponential is irrelevant, we consider

$$
\varphi_{t}(z)=t^{-d / 2} \exp \left(-|z|^{2} / t\right)
$$

where we abuse notation and keep the same name. Now this is the Schwartz function $z \mapsto \exp \left(-z^{2}\right)$ scaled by a factor of $\sqrt{t}$, and so part (i) with $\ell=0$ follows from Remark 9.

Now

$\partial_{t}\left[t^{-d / 2} \exp \left(-|z|^{2} / t\right)\right]=(-d / 2) t^{-d / 2-1} \exp \left(-|z|^{2} / t\right)+t^{-d / 2} \exp \left(-|z|^{2} / t\right)|z|^{2} t^{-2}$.

The first term is treated as above, now having the additional prefactor $t^{-1}=(\sqrt{t})^{-2}$.

We write the second term as

$$
t^{-d / 2-1} \exp \left(-|z|^{2} / t\right)\left(\frac{|z|}{\sqrt{t}}\right)^{2}=t^{-d / 2-1} \phi_{0}^{\sqrt{t}}(z)
$$

where $\phi(s):=s^{2} \exp \left(-s^{2}\right)$ is Schwartz. By Remark 9 part (i) with $\ell=1$ is proven.

For the second statement

$$
\begin{aligned}
Y_{p} \mathrm{p}_{t}(p, q)= & Y_{p}\left[t^{-d / 2} \exp \left(-\frac{d(p, q)^{2}}{4 t}\right) \Phi(p, q)\right] \\
= & -\frac{1}{2} t^{-d / 2-1} \exp \left(-\frac{d(p, q)^{2}}{4 t}\right) Y_{p}\left[d^{2}(p, q)\right] \Phi(p, q) \\
& +\frac{1}{2} t^{-1} \exp \left(-\frac{d(p, q)^{2}}{4 t}\right) Y_{p}[\Phi(p, q)] .
\end{aligned}
$$

The first term has worse blowup in $t$ and the factor $1 / 4$ in the exponential is irrelevant, so it is enough to consider $f(z) g(z)$ where

$$
\begin{aligned}
& f(z):=t^{-d / 2-1} \exp \left(-|z|^{2} / t\right) \\
& g(z):=\left.Y_{p}\left[d^{2}(p, \cdot)\right]\right|_{.=\exp _{p}(z)}
\end{aligned}
$$


Now for a multiindex $k$

$$
D^{k}[f(z) g(z)]=\sum_{\beta \leq k} c_{\beta, k} D^{k-\beta} f(z) D^{\beta} g(z) .
$$

By Lemma 45

$$
\begin{array}{ll}
\left|D^{\beta} g(z)\right| & \lesssim|z| \text { if }|\beta|=0 \\
\left|D^{\beta} g(z)\right| \lesssim 1 & \text { else. }
\end{array}
$$

and by Lemma 47

$$
\left|D^{k-\beta} f(z)\right| \lesssim t^{-d / 2-1-|k-\beta| / 2}\left(\frac{|z|}{t^{1 / 2}}\right)^{|k-\beta|} \exp \left(-|z|^{2} / t\right)
$$

Hence for $|k-\beta| \leq|k|-1$

$$
\begin{aligned}
\left|f^{k-i}(z) g^{(i)}(z)\right| & \lesssim t^{-d / 2-1-|k-\beta| / 2}\left(\frac{|z|}{t^{1 / 2}}\right)^{k-i} \exp \left(-|z|^{2} / t\right) \\
& \lesssim t^{-d / 2-1 / 2-|k| / 2}\left(\frac{|z|}{t^{1 / 2}}\right)^{k-i} \exp \left(-|z|^{2} / t\right)
\end{aligned}
$$

For $|k-\beta|=|k|$ we have $|\beta|=0$ and then

$$
\begin{aligned}
\left|D^{k-\beta} f(z) D^{\beta} g(z)\right| & \lesssim t^{-d / 2-1-k / 2}\left(\frac{d}{t^{1 / 2}}\right)^{k} \exp \left(-|z|^{2} / t\right)|z| \\
& \lesssim t^{-d / 2-1 / 2-k / 2}\left(\frac{d}{t^{1 / 2}}\right)^{k+1} \exp \left(-|z|^{2} / t\right)
\end{aligned}
$$

The second statement then follows, since $s \mapsto s^{j} \exp \left(-s^{2}\right)$ is a Schwartz function, for any $j \geq 0$.

The third statement follows in a similar fashion from Lemmas 45 and 46.

Lemma 45 Let $Y_{p} \in T_{p} M$ act on the first component of $d^{2}$ as follows

$$
g(z):=Y_{p}\left[d(p, \cdot)^{2}\right] \mid \cdot=\exp _{p}(z)
$$

Then

$$
\begin{aligned}
|g(z)| & \lesssim|z|\left|Y_{p}\right| \\
\left|D^{\beta} g(z)\right| & \lesssim\left|Y_{p}\right|, \quad \text { for any multiindex } \beta .
\end{aligned}
$$

Proof Since $(p, q) \mapsto d^{2}(p, q)$ is smooth, we only need to show $g(z) \lesssim|z|\left|Y_{p}\right|$. Let $h(q)=Y_{p}\left[d^{2}(p, q)\right]$. 
Fix $q$ and take coordinates $\exp _{q}^{-1}$. Then

$$
\begin{aligned}
|h(q)| & =\left|Y_{p^{i}}^{i} \partial_{r^{i}}\right| d^{2}\left(\exp _{q}(r), q\right) \mid \\
& =\left.\left|Y_{p}^{i} \partial_{r^{i}}\right| r\right|^{2} \mid \\
& =\left|Y_{p}^{i} 2 r_{i}\right| \\
& \lesssim\left|Y_{p}\right| d(p, q) .
\end{aligned}
$$

Then $|g(z)|=\left|h\left(\exp _{p}(z)\right)\right| \lesssim\left|Y_{p}\right||z|$ as desired.

Lemma 46 For $Y_{p} \in T_{p} M$ and a vector field $Z$ let

$$
g(z):=Y_{p}\left[Z\left[d(\bullet, \cdot)^{2}\right]\right] \mid \cdot=\exp _{p}(z)
$$

Then, for any multi-index $\beta$,

$$
\left|D^{\beta} g(z)\right| \lesssim Z\left|Y_{p}\right|
$$

Proof This follows from the fact that $(p, q) \mapsto d^{2}(p, q)$ is smooth.

Lemma 47 For any multiindex $k$

$$
\left|D_{z}^{k} \exp \left(-|z|^{2} / t\right)\right| \lesssim_{k} t^{-|k| / 2}\left(\frac{|z|}{t^{1 / 2}}\right)^{|k|} \exp \left(-|z|^{2} / t\right)
$$

Proof This can be verified using the Faa di Bruno formula.

\section{Fixpoint argument}

The following lemma follows from a direct application of the definition of modelled distributions.

Lemma 48 Define "multiplication by $\Xi$ " as the vector bundle morphism $m \Xi: \mathcal{W} \rightarrow$ $\mathcal{V}$ satisfying

$$
\begin{aligned}
m^{\Xi}(\mathbf{1}) & :=\boldsymbol{\Xi} \\
m^{\boldsymbol{\Xi}}(\mathcal{I}[\boldsymbol{\Xi}]) & :=\mathcal{I}[\boldsymbol{\Xi}] \boldsymbol{\Xi} \\
m^{\boldsymbol{\Xi}}(\omega) & :=\omega \boldsymbol{\Xi} \quad p \in M, \omega \in T_{p}^{*} M .
\end{aligned}
$$

If $f \in \mathcal{D}_{T}^{\gamma, \gamma_{0}}(M, \mathcal{W})$ then $m(f) \in \mathcal{D}^{\gamma, \gamma_{0}}(M, \mathcal{V})$ and for $\mathfrak{N}>0$

$$
\left\|m^{\Xi}(f)\right\|_{\mathcal{D}_{T}^{\gamma, \gamma_{0}, \mathfrak{N}}(M, \mathcal{V})}=\|f\|_{\mathcal{D}_{T}^{\gamma, \gamma_{0}, \mathfrak{N}}(M, \mathcal{W})} .
$$


Theorem 49 Let $u_{0} \in C^{\infty}\left(\mathbb{R}^{2}\right)$. Define $v_{t}:=P_{t} u_{0}$ and lift it to the regularity structure as

$$
V_{t}(p):=\mathbf{1} v_{t}(p)+\mathcal{I}[\boldsymbol{\Xi}] 0+\left.d\right|_{p} v_{t}
$$

Let $(\xi, Z)$ be given as in Definition 35 and let $\Pi_{p}^{t, \mathcal{G}}, \Gamma_{p}^{t, \mathcal{G}} \leftarrow q$ be the corresponding models given by Lemma $36, \mathcal{G}=\mathcal{V}, \mathcal{W}$. Let $\alpha \in(-4 / 3,-1), \gamma_{0}:=\alpha / 2+1$ and $\gamma \in(4 / 3,2 \alpha+4)$. Then there exists $T>0$ and a unique $u \in \mathscr{D}_{T}^{\gamma^{\gamma} \gamma_{0}}(M, \mathcal{W})$ such that on $[0, T]$

$$
u_{t}=\mathcal{K}_{t}\left[m^{\Xi}(u)\right]+V_{t}
$$

Proof We follow a standard fixpoint argument. Denote

$$
B(R, \mathfrak{N}):=\left\{f \in \mathcal{D}_{T}^{\gamma, \gamma_{0}}(M, \mathcal{W}):\|f-V\|_{\mathcal{D}_{T}^{\gamma, \gamma_{0}, \mathfrak{N}}(M, \mathcal{W})} \leq R\right\}
$$

Denote for $f \in B(R, \mathfrak{N})$

$$
\Phi(f):=\mathcal{K}_{t}\left[m^{\Xi}(f)\right]+V_{t}
$$

Claim: for any $\mathfrak{N}>0$, there is $R>0$ such that $\Phi(B(R, \mathfrak{N})) \subset B(R, \mathfrak{N})$.

Indeed, by Theorem 38 and Lemma 48, for a constant $c>0$ possibly changing from line to line,

$$
\begin{aligned}
& \|\Phi(f)-V\|_{\mathcal{D}_{T}^{\gamma, \gamma_{0}, \mathfrak{N}}{ }_{(M, \mathcal{W})}}=\left\|\mathcal{K}_{t}\left[m^{\Xi}(f)\right]\right\|_{\mathcal{D}_{T}^{\gamma, \gamma_{0}, \mathfrak{N}}{ }_{(M, \mathcal{W})}} \\
& \leq c\left\|m^{\Xi}(f)\right\|_{\mathcal{D}_{T}^{\gamma-4 / 3, \gamma_{0}, \mathfrak{N}}(M, \mathcal{V})}\left(T^{\varepsilon}+T^{\varepsilon} \mathfrak{N}+\frac{1}{\mathfrak{N}}\right) \\
& =c\|f\|_{\mathcal{D}_{T}^{\gamma-4 / 3-\alpha, \gamma_{0}, \mathfrak{N}}(M, \mathcal{V})}\left(T^{\varepsilon}+T^{\varepsilon} \mathfrak{N}+\frac{1}{\mathfrak{N}}\right) \\
& \leq c\|f\|_{\mathcal{D}_{T}^{\gamma, \gamma_{0}, \mathfrak{N}}(M, \mathcal{V})}\left(T^{\varepsilon}+T^{\varepsilon} \mathfrak{N}+\frac{1}{\mathfrak{N}}\right),
\end{aligned}
$$

since $\alpha>-4 / 3$. Hence for $T$ small enough and $\mathfrak{N}$ large enough, $\Phi(B(R, \mathfrak{N})) \subset$ $B(R, \mathfrak{N})$, for any $R>0$.

Let us show that $\Phi$ is a contraction on $B(R, \mathfrak{N})$ : for any $f, f^{\prime} \in B(R, \mathfrak{N})$,

$$
\begin{aligned}
& \left\|\Phi(f)-\Phi\left(f^{\prime}\right)\right\|_{\mathcal{D}_{T}^{\gamma, \gamma_{0}, \mathfrak{N}}{ }_{(M, \mathcal{W})}}=\left\|\mathcal{K}_{t}\left[m^{\Xi}\left(f-f^{\prime}\right)\right]\right\|_{\mathcal{D}_{T}^{\gamma, \gamma_{0}, \mathfrak{N}}{ }_{(M, \mathcal{W})}} \\
& \leq c\left\|m^{\Xi}\left(f-f^{\prime}\right)\right\|_{\mathcal{D}_{T}^{\gamma-4 / 3, \gamma_{0}, \mathfrak{N}}(M, \mathcal{V})}\left(T^{\varepsilon}+T^{\varepsilon} \mathfrak{N}+\frac{1}{\mathfrak{N}}\right) \\
& =c\left\|f-f^{\prime}\right\|_{\mathcal{D}_{T}^{\gamma-4 / 3-\alpha, \gamma_{0}, \mathfrak{N}}(M, \mathcal{V})}\left(T^{\varepsilon}+T^{\varepsilon} \mathfrak{N}+\frac{1}{\mathfrak{N}}\right)
\end{aligned}
$$




$$
\leq c\left\|f-f^{\prime}\right\|_{\mathcal{D}_{T}^{\gamma, \gamma_{0}, \mathfrak{N}}(M, \mathcal{V})}\left(T^{\varepsilon}+T^{\varepsilon} \mathfrak{N}+\frac{1}{\mathfrak{N}}\right)
$$

Hence for $T$ small enough and $\mathfrak{N}$ large enough, $\Phi$ is a contraction on $B(R, \mathfrak{N})$ for any $R>0$. We therefore get unique existence of a solution for small $T>0$.

To apply this theorem to white noise forcing, the only ingredient missing is "lifting" it to a model (Definition 35). This is done in the next section.

\section{The Gaussian model}

Let $\xi$ be a white noise on $M$. We recall that $\xi$ is a Gaussian process associated to the Hilbert space $L^{2}\left(M, \operatorname{vol}_{M}\right)$, on a probability space $(\Omega, \mathcal{B}, \mathbb{P})$.

Lemma 50 There exists a realization of $\xi$ such that almost surely for any $\alpha<-1$, $\xi \in C^{\alpha}(M)$.

Proof For any coordinate chart $\psi$ defined on an open subset $\mathcal{U} \subset M$, and $\rho$ a positive function with support in $\mathcal{U}, \xi_{\mathcal{U}}=\rho \circ \psi^{-1} \psi_{*} \xi$ is a Gaussian process associated to the Hilbert space $L^{2}\left(\mathbb{R}^{2}, \rho^{2} \circ \psi^{-1} \operatorname{det}\left(g \circ \psi^{-1}\right)\right)$. Note that $\xi_{\mathcal{U}}$ has the same law as $\eta v$, with $\eta:=\rho \circ \psi^{-1} \sqrt{g \circ \psi^{-1}}$ and $v$ a white-noise on $\mathbb{R}^{d}$. According to [9, Lemma 10.2] $v$ has a version which is almost surely in $C^{\alpha}\left(\mathbb{R}^{d}\right)$ and hence $\xi_{\mathcal{U}} \in C^{\alpha}\left(\mathbb{R}^{d}\right)$.

Let now $\left(\rho_{i}\right)_{1 \leq i \leq n}$ be a partition of unity subordinated to an atlas $\left(\mathcal{U}_{i}, \psi_{i}\right)_{1 \leq i \leq n}$. Then, there is a realization of $\left(\xi_{\mathcal{U}_{i}}\right)_{1 \leq i \leq n}$ such that almost surely for all $\alpha<-1, i \in$ $\{1, \ldots, n\}, \xi_{\mathcal{U}_{i}} \in C^{\alpha}\left(\mathbb{R}^{2}\right)$. Then, $\sum_{i=1}^{n} \psi_{i}^{*} \xi_{\mathcal{U}_{i}}$ is a realization of $\xi$ belonging almost surely to $C^{\alpha}(M)$.

Thanks to this realization, we can already define the transport map used in the following Lemma (point (i)).

Lemma 51 Let $\xi$ be the white noise on $M$ and $Z_{p}^{t}, p \in M, t \in[0, T]$ be a collection of random distributions on $M$ such that for some $\alpha \in(-4 / 3,-1)$, some $\kappa, \delta>0$,

(i) $Z_{q}^{t}(z)=Z_{p}^{t}(z)+\int_{0}^{t}\left\langle p_{t-r}(p, \cdot)-p_{t-r}(q, \cdot), \xi\right\rangle d r \xi(z)$,

$$
\sup _{p \in M, 0 \leq t, s \leq T, \lambda \in(0,1], \varphi \in \mathcal{B}_{p_{p} M}^{-[\alpha], \delta}} \lambda^{-2(2 \alpha+2)-\kappa} \mathbb{E}\left[\left\langle Z_{p}^{0}, \varphi_{p}^{\lambda}\right\rangle^{2}+|t-s|^{-\kappa}\left\langle Z_{p}^{t}-Z_{p}^{s}, \varphi_{p}^{\lambda}\right\rangle^{2}\right]<\infty,
$$

(iii) for any $\varphi \in C^{\infty}(M), t \in[0, T], p \in M:\left\langle Z_{p}^{t}, \varphi\right\rangle$ is in the second Wiener chaos.

Then, there is a version of $Z$ and a constant $h>0$ such that a.s.

$$
\sup _{p \in M, 0 \leq t, s \leq T, \lambda \in(0,1], \varphi \in \mathcal{B}_{T_{p} M}^{-[\alpha], \delta}} \lambda^{-(2 \alpha+2)}\left(\left|\left\langle Z_{p}^{0}, \varphi_{p}^{\lambda}\right\rangle\right|+|t-s|^{-h}\left|\left\langle Z_{p}^{t}-Z_{p}^{s}, \varphi_{p}^{\lambda}\right\rangle\right|\right)<\infty .
$$


Proof For $t>s \geq 0$, define for a chart $(\Psi, \mathcal{U})$

$$
\begin{aligned}
\bar{Z}_{x}^{s, t} & :=\Psi_{*}\left(Z_{\Psi^{-1}(x)}^{t}-Z_{\Psi^{-1}(x)}^{s}\right), \quad x \in \Psi(\mathcal{U}) \\
\bar{\xi} & :=\Psi_{*} \xi .
\end{aligned}
$$

Note that $\bar{Z}_{x}^{s, t}, x \in \Psi(\mathcal{U})$ and $\bar{\xi}$ are elements of $D^{\prime}(\Psi(\mathcal{U}))$. Then

$$
\begin{aligned}
\left\langle\bar{Z}_{y}^{s, t}, \varphi\right\rangle & =\left\langle Z_{\Psi^{-1}(y)}^{s, t}, \varphi \circ \Psi\right\rangle \\
& =\left\langle Z_{\Psi^{-1}(x)}^{s, t}+\left\langle\int_{s}^{t}\left[\mathrm{p}_{t-r}\left(\Psi^{-1}(x), \cdot\right)-\mathrm{p}_{t-r}\left(\Psi^{-1}(y), \cdot\right)\right] d r, \xi\right) \xi, \varphi \circ \Psi\right\rangle \\
& =\left\langle\bar{Z}_{x}^{s, t}+\left\langle\int_{s}^{t}\left[\mathrm{p}_{t-r}\left(\Psi^{-1}(x), \cdot\right)-\mathrm{p}_{t-r}\left(\Psi^{-1}(y), \cdot\right)\right] d r, \xi\right) \bar{\xi}, \varphi\right\rangle \\
& =\left\langle\bar{Z}_{x}^{s, t}+S^{s, t}(x \leftarrow y) \bar{\xi}, \varphi\right\rangle,
\end{aligned}
$$

where we denote $S^{s, t}(x \leftarrow y):=\left\langle\int_{s}^{t}\left[\mathrm{p}_{t-r}\left(\Psi^{-1}(x), \cdot\right)-\mathrm{p}_{t-r}\left(\Psi^{-1}(y), \cdot\right)\right] d r, \xi\right\rangle$.

Define the regularity structure and model (in the stronger sense of [9])

$$
\begin{aligned}
\mathcal{T} & :=\operatorname{span}\{\boldsymbol{\Xi}\} \oplus \operatorname{span}\{\mathcal{I}[\boldsymbol{\Xi}] \boldsymbol{\Xi}\} \oplus \operatorname{span}\{\mathbf{1}\} \\
\Pi_{x}^{s, t} \boldsymbol{\Xi} & :=\bar{\xi} \\
\left.\Pi_{x}^{s, t} \mathcal{I}[\boldsymbol{\Xi}] \boldsymbol{\Xi}\right] & :=\bar{Z}_{x}^{s, t} \\
\Pi_{x}^{s, t} \mathbf{1} & :=1 \\
\Gamma_{x \leftarrow y}^{s, t} \boldsymbol{\Xi} & :=\boldsymbol{\Xi} \\
\Gamma_{x \leftarrow y}^{s, t} \mathcal{I}[\boldsymbol{\Xi}] \boldsymbol{\Xi} & :=\mathcal{I}[\boldsymbol{\Xi}] \boldsymbol{\Xi}+S^{s, t}(x \leftarrow y) \boldsymbol{\Xi} \\
\Gamma_{x \leftarrow y}^{s, t} \mathbf{1} & :=\mathbf{1},
\end{aligned}
$$

and the sector (in the sense of [9, Definition 2.5])

$$
V:=\operatorname{span}\{\boldsymbol{\Xi}\} \oplus \operatorname{span}\{\mathcal{I}[\boldsymbol{\Xi}] \boldsymbol{\Xi}\}
$$

One can then apply [9, Proposition 3.32] to get for every compactum $K_{-} \subset \subset K \subset$ $\Psi(U)$, and $^{8} \varphi \in \mathcal{B}_{\mathbb{R}^{d}}^{r, 1}, r:=-[\alpha]$, with $\operatorname{supp} \varphi \subset K_{-}$,

$$
\begin{aligned}
\left|\left\langle\bar{Z}_{x}^{s, t}, \varphi_{x}^{\lambda}\right\rangle\right| \lesssim & \lambda^{2 \alpha+2}|| \Pi^{s, t}, \Gamma^{s, t} \|_{V, \mathcal{K}} \\
\lesssim & \lambda^{2 \alpha+2}\left(1+\left\|\Gamma^{s, t}\right\|_{V, \mathcal{K}}\right) \\
& \times \sup _{a=\alpha, 2 \alpha+2} \sup _{\tau \in V_{a}} \sup _{n \geq 0} \sup _{z \in \text {-dyadics } \cap \mathcal{K}} \lambda^{(2 \alpha+2) n} \frac{\left|\left\langle\Pi_{z}^{s, t} \tau, \varphi_{z}^{n}\right\rangle\right|}{|\tau|} \\
\lesssim & \lambda^{2 \alpha+2}\left(1+\sup _{x, y \in \mathbb{R}^{d}}|x-y|^{-(\alpha+2)} S^{s, t}(x \leftarrow y)\right)
\end{aligned}
$$

$\overline{8}$ In the notation of [9, Proposition 3.32], $\varphi_{z}^{n}$ stands for $2^{-n d / 2} \varphi_{z}^{2^{-n}}$. 


$$
\times \sup _{n \geq 0} \sup _{z \in \text { n-dyadics } \cap \mathcal{K}}\left(2^{(2 \alpha+2) n}\left\langle\bar{Z}_{z}^{s, t}, \varphi_{z}^{n}\right\rangle+2^{\alpha n}\left\langle\bar{Z}_{z}^{s, t}, \varphi_{z}^{n}\right\rangle\left\langle\bar{\xi}, \varphi_{z}^{n}\right\rangle\right) .
$$

Then, for $q \in \mathbb{N}$ large enough and any $\delta>0$, using (iii), equivalence of moments and then (i)

$$
\begin{aligned}
& \mathbb{E}\left[\sup _{n \geq 0} \sup _{|z|<\delta, z \in \text {-dyadics }}\left(2^{(2 \alpha+2) n q}\left|\left\langle\bar{Z}_{z}^{s, t}, \varphi_{z}^{2^{-n}}\right\rangle\right|^{q}+2^{\alpha n q}\left|\left\langle\bar{Z}_{z}^{s, t}, \varphi_{z}^{2^{-n}}\right\rangle\right|^{q}\left|\left\langle\bar{\xi}, \varphi_{z}^{2^{-n}}\right\rangle\right|^{q}\right)\right] \\
& \lesssim \sum_{n \geq 0} 2^{2 n}\left(2^{(2 \alpha+2) n q} \sup _{|z|<2 \delta} \mathbb{E}\left[\left|\left\langle\bar{Z}_{z}^{s, t}, \varphi_{z}^{2^{-n}}\right\rangle\right|^{2}\right]^{\frac{q}{2}}+2^{\alpha n q} \sup _{|z|<2 \delta} \mathbb{E}\left[\left|\left\langle\bar{Z}_{z}^{s, t}, \varphi_{z}^{2^{-n}}\right\rangle\right|^{2}\left|\left\langle\bar{\xi}, \varphi_{z}^{2^{-n}}\right\rangle\right|^{2}\right]^{\frac{1}{2 q}}\right) \\
& \quad \lesssim|t-s|^{\frac{q \kappa}{2}} .
\end{aligned}
$$

Let now $\left(\Psi_{i}, \mathcal{U}_{i}\right)$ be a finite atlas with subordinate partition of unity $\phi_{i}$ and $\delta>0$ be the radius of injectivity of $M$. Then for $s, t \in[0, T], p \in M, \varphi \in \mathcal{B}_{T_{p} M}^{r, \delta}$

$$
\left\langle Z_{p}^{s, t}, \varphi_{p}^{\lambda}\right\rangle=\sum_{i: \phi_{i} \varphi_{p}^{\lambda} \neq 0}\left\langle Z_{p}^{s, t}, \phi_{i} \varphi_{p}^{\lambda}\right\rangle
$$

Now for $\lambda$ small enough, $\phi_{i} \varphi_{p}^{\lambda} \not \equiv 0$ implies that $\operatorname{supp} \varphi_{p}^{\lambda} \subset \mathcal{U}_{i}$ and in particular $p \in \mathcal{U}_{i}$. Hence

$$
\left\langle Z_{p}^{s, t}, \varphi_{p}^{\lambda}\right\rangle=\sum_{i: \phi_{i} \varphi_{p}^{\lambda} \neq 0}\left\langle\bar{Z}_{\Psi^{i}(p)}^{s, t ; i},\left(\phi_{i} \varphi_{p}^{\lambda}\right) \circ \Psi_{i}^{-1}\right\rangle
$$

where $\bar{Z}_{x}^{s, t ; i}:=\left(\Psi_{i}\right)_{*}\left(Z_{\Psi_{i}^{-1}(x)}^{t}-Z_{\Psi_{i}^{-1}(x)}^{s}\right)$. We can apply Remark 7 to $\left(\phi_{i} \varphi_{p}^{\lambda}\right) \circ \Psi_{i}^{-1}$ and can estimate, using (15),

$$
\begin{aligned}
\left|\left\langle Z_{p}^{s, t}, \varphi_{p}^{\lambda}\right\rangle\right| \lesssim & \lambda^{2 \alpha+2}\left(1+\sup _{x, y \in \mathbb{R}^{d}}|x-y|^{-(\alpha+2)} S^{s, t}(x \leftarrow y)\right) \\
& \times \sum_{i} \sup _{n \geq 0} \sup _{z \in \text {-dyadics } \cap \mathcal{K}_{i}}\left(2^{(2 \alpha+2) n}\left\langle\bar{Z}_{z}^{s, t ; i}, \varphi_{z}^{2^{-n}}\right\rangle+2^{\alpha n}\left\langle\bar{Z}_{z}^{s, t ; i}, \varphi_{z}^{2^{-n}}\right\rangle\left\langle\bar{\xi}^{i}, \varphi_{z}^{n}\right\rangle\right),
\end{aligned}
$$

here for every $i, \mathcal{K}_{i}$ is some compactum satisfying $\Psi_{i}\left(\operatorname{supp} \phi_{i}\right) \subset \subset \mathcal{K}_{i} \subset \Psi_{i}\left(\mathcal{U}_{i}\right)$. Then, by (16),

$$
\mathbb{E}\left[\sup _{p \in M, \lambda \in(0,1], \varphi \in \mathcal{B}_{T p M}^{r, \delta}} \lambda^{-(2 \alpha+2)}\left|\left\langle Z_{p}^{s, t}, \varphi_{p}^{\lambda}\right\rangle\right|^{q}\right] \lesssim|t-s|^{q \kappa / 2} .
$$

Let us formulate a setting where we can apply Kolmogorov's continuity theorem in time. Endow the linear space $\chi$ of maps $Y: M \rightarrow \mathcal{D}^{\prime}(M)$, such that for any $p \in M$, 
$\operatorname{supp}\left(Y_{p}\right) \subset B(p, \delta / 2)$, with the norm

$$
\|Y\|:=\sup _{p \in M, \lambda \in(0,1), \varphi \in \mathcal{B}_{T_{p} M}^{r, \delta}} \lambda^{-(2 \alpha+2)}\left|\left\langle Y_{p}, \varphi_{p}^{\lambda}\right\rangle\right|
$$

and consider the Banach space $\chi_{2 \alpha+2}=\{Y \in \chi:\|Y\|<\infty\}$. Using Remark 7, we apply this to

$$
Y_{p}^{t}:=Z_{p}^{t} \rho_{p}
$$

Here $\rho_{p}:=\rho \circ \exp _{p}^{-1}$, with $\rho$ smooth, supp $\rho \subset B_{T_{p} M}\left(0_{p}, \delta / 2-\epsilon\right)$ for some $\epsilon>0$ small enough.

Then, from the argument before, for any $s, t \geq 0$ and $q$ large enough, we have

$$
\mathbb{E}\left\|Y^{t}-Y^{s}\right\|^{q} \lesssim|t-s|^{\frac{\kappa q}{2}}
$$

The result now follows from the Kolmogorov continuity theorem.

A simple way to define $Z_{p}^{t}$ is here to recenter the terms involving one product of distributions by their mean; this is an instance of a Wick product, see for instance [8]. For any $t>0$, the heat kernel and the heat operator are denoted respectively by $\mathrm{p}_{t}: M^{2} \rightarrow \mathbb{R}$ and $P_{t}$, and we write for $p \in M, \mathrm{q}_{t}(p)=\mathrm{p}_{t}(p, p)$. According to Lemma 50 and Theorem 43, we can consider $P_{t}(\xi)$ as a function and the map $t \in \mathbb{R}_{>0} \mapsto P_{t}(\xi) \in C^{\infty}(M)$ is continuous.

We set for any $p \in M, t \in \mathbb{R}_{\geq 0}$ and any function $\varphi \in C^{\infty}(M)$,

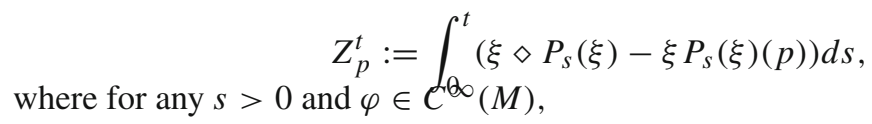

$$
\left\langle\xi \diamond P_{S}(\xi), \varphi\right\rangle:=\left\langle\xi, \varphi P_{S}(\xi)\right\rangle-\mathbb{E}\left[\left\langle\xi, \varphi P_{S}(\xi)\right\rangle\right]
$$

Note that for any $s>0$,

$$
\xi \diamond P_{s} \xi=P_{S}(\xi) \xi-\mathrm{q}_{s}
$$

For any $t \geq 0$, let us consider the operator $K_{t}=\int_{0}^{t} P_{s} d s$ and for any $p, q \in M$ with $p \neq q$, set $\mathrm{k}_{t}(p, q)=\int_{0}^{t} \mathrm{p}_{s}(p, q) d s$. Let us note that the operator

$$
K_{t}^{2}=\int_{0 \leq s, s^{\prime} \leq t} P_{s+s^{\prime}} d s d s^{\prime}=\int_{0}^{2 t} s P_{s} d s
$$

has a continuous kernel according to Theorem 43 , that we shall denote $\mathrm{k}_{2, t}$. 
Remark 52 Note that, in (17) we subtract a function depending on space. This is different than the renormalization in the flat case, [9, Section 9.1], where just a constant is subtracted. of

This could be avoided, by realizing that the only factor contributing to the blowup

$$
\int_{0}^{t} d s \mathrm{q}_{s}(\cdot)
$$

is, by Theorem 43,

$$
\int_{0}^{t} d s s^{-1} \Phi_{0}(\cdot, \cdot)=\int_{0}^{t} d s s^{-1}
$$

which is independent of space. Since we do not need the renormalization to be independent of space (or time, for that matter), we do not pursue this.

Proposition 53 For any $t \in \mathbb{R}_{\geq 0}$, almost surely for any $p \in M$ and $\varphi \in C^{\infty}(M)$, $\left\langle Z_{p}^{t}, \varphi\right\rangle$ is well-defined and there exists a modification of the process given by $\left(\left\langle Z_{p}^{t}, \varphi\right\rangle\right)_{p \in M, \varphi \in C^{\infty}(M), t \geq 0}$ such that almost surely (13) holds true. ${ }^{9}$

Proof of Proposition 53 It is enough to prove the assumption of Lemma 51. Let us fix $p \in M$ and $T>0$. Let $\delta$ be the radius of injectivity of $M$.

Let us first check that for any $\varphi \in C^{\infty}(M), Z_{x}^{t}(\varphi)$, is well defined for $0 \leq t \leq T$. Therefor, let us recall - see Theorem 43 - that

$$
L:=\sup _{p \in M, s \in(0,2 T]} s \mathrm{q}_{s}(p)<\infty
$$

The Wick formulas imply for any $0<s<T$,

$\operatorname{Var}\left(\left\langle\xi, \varphi P_{s} \xi\right\rangle\right)=\int \mathrm{q}_{2 s}(z) \varphi(z)^{2} d z+\int \mathrm{p}_{s}\left(z, z^{\prime}\right)^{2} \varphi(z) \varphi\left(z^{\prime}\right) d z d z^{\prime} \leq 2 \operatorname{vol}(M) L\|\varphi\|_{\infty}^{2} s^{-1}$

It follows that

$$
\mathbb{E}\left[\int_{0}^{t}\left|\left\langle P_{s} \xi \diamond \xi, \varphi\right\rangle\right| d s\right] \leq \int_{0}^{t} \operatorname{Var}\left(\left\langle\xi, \varphi P_{s} \xi\right\rangle\right)^{1 / 2} d s<\infty .
$$

Besides, $\mathbb{E}\left(\langle\xi, \varphi\rangle^{2} P_{s} \xi(p)^{2}\right)=q_{2 s}(p)\|\varphi\|_{2}^{2}+P_{s}(\varphi)(p)^{2} \leq s(L+1)\|\varphi\|_{\infty}^{2}$, so that $Z_{p}^{t}(\varphi)$ is well defined. We shall now prove that for any $\kappa<0$,

$$
\sup _{t \in[0, T], p \in M, \varphi \in \mathcal{B}_{T_{p} M}^{2, \delta}} \lambda^{-2 \kappa} \mathbb{E}\left[\left(Z_{p}^{t}\left(\varphi_{p}^{\lambda}\right)\right)^{2}\right]<\infty
$$

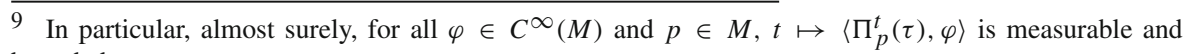
bounded. 
which together with Lemma 50 shall yield the claim. We fix now $\kappa<0$. Let us first prove that the expectation of the second integrand in $\Pi_{p}^{t}(\mathcal{I}(\Xi) \Xi)$ is almost surely of homogeneity $\kappa$. Indeed, according to Theorem 43, there exists $C_{T}>0$, such that, for all $0<t<T, p, q \in M$, with $p \neq q$,

$$
\begin{aligned}
\left|\mathrm{k}_{t}(p, q)\right| & \lesssim C_{T}+\int_{0}^{T} e^{-\frac{d(p, q)^{2}}{4 s}} \frac{d s}{s}=C_{T}+\int_{\frac{d(p, q)^{2}}{4 T}}^{+\infty} e^{-v} \frac{d v}{v} \\
& \lesssim|\log (d(p, q))| .
\end{aligned}
$$

Since

$$
\mathbb{E}\left[\left\langle\xi P_{s} \xi(p), \varphi\right\rangle\right]=P_{s} \varphi(p),
$$

it follows that for any $\kappa<0$,

$$
\sup _{p \in M, \varphi \in \mathcal{B}_{T_{p} M}^{2, \delta}}\left|\mathbb{E}\left[\left\langle\xi K_{t} \xi(p), \varphi_{p}^{\lambda}\right\rangle\right]\right|=\sup _{p \in M, \varphi \in \mathcal{B}_{T_{p} M}^{2, \delta}}\left|K_{t}\left(\varphi_{p}^{\lambda}\right)(p)\right| \leq C_{T} \lambda^{\kappa}
$$

Setting $I_{p, s}=\xi P_{s} \xi-\xi P_{s} \xi(p)$ and $: I_{p, s}:=I_{p, s}-\mathbb{E}\left[I_{p, s}\right],{ }^{10}$ it remains to estimate

$$
\left\langle: Z_{p}^{t}:, \varphi_{p}^{\lambda}\right\rangle:=\left\langle Z_{p}^{t}, \varphi_{p}^{\lambda}\right\rangle-\mathbb{E}\left[\left\langle Z_{p}^{t}, \varphi_{p}^{\lambda}\right\rangle\right]=\int_{0}^{t}\left\langle: I_{p, s}:, \varphi_{p}^{\lambda}\right\rangle d s
$$

For any $\varphi \in \mathcal{B}_{T_{p} M}^{2, \delta}, s, s^{\prime} \geq 0$,

$$
\begin{aligned}
\mathbb{E}[\langle: & \left.\left.I_{p, s}:, \varphi_{p}^{\lambda}\right\rangle\left\langle: I_{p, s^{\prime}}:, \varphi_{p}^{\lambda}\right\rangle\right] \\
= & \int_{M}\left(\mathrm{p}_{s+s^{\prime}}(q, q)+\mathrm{p}_{s+s^{\prime}}(p, p)-2 \mathrm{p}_{s+s^{\prime}}(q, p)\right) \varphi_{p}^{\lambda}(q)^{2} d q \\
& +\int_{M^{2}}\left(\mathrm{p}_{s}\left(q, q^{\prime}\right)-\mathrm{p}_{s}\left(p, q^{\prime}\right)\right)\left(\mathrm{p}_{s^{\prime}}\left(q, q^{\prime}\right)-\mathrm{p}_{s^{\prime}}(p, q)\right) \varphi_{p}^{\lambda}(q) \varphi_{p}^{\lambda}\left(q^{\prime}\right) d q d q^{\prime} .
\end{aligned}
$$

and

$$
\begin{aligned}
\mathbb{E}\left[\left\langle: Z_{p}^{t}:, \varphi_{p}^{\lambda}\right\rangle^{2}\right]= & \int_{M}\left(\mathrm{k}_{2, t}(q, q)+\mathrm{k}_{2, t}(p, p)-2 \mathrm{k}_{2, t}(q, p)\right) \varphi_{p}^{\lambda}(q)^{2} d q \\
& +\int_{M^{2}}\left(\mathrm{k}_{t}\left(q, q^{\prime}\right)-\mathrm{k}_{t}\left(p, q^{\prime}\right)\right)\left(\mathrm{k}_{t}\left(q, q^{\prime}\right)-\mathrm{k}_{t}(p, q)\right) \varphi_{p}^{\lambda}(q) \varphi_{p}^{\lambda}\left(q^{\prime}\right) d q d q^{\prime} \\
\leq & 2 \int_{M}\left(\mathrm{k}_{2, t}(q, q)+\mathrm{k}_{2, t}(p, p)-2 \mathrm{k}_{2, t}(q, p)\right) \varphi_{p}^{\lambda}(q)^{2} d q,
\end{aligned}
$$

10 Where we denote the distribution $\left\langle\mathbb{E}\left[I_{p, s}\right], \varphi\right\rangle:=\mathbb{E}\left[\left\langle I_{p, s}, \varphi\right\rangle\right]$. 
where the second line follows from the Cauchy-Schwarz inequality. It follows from Lemma 54 below, that there exists $C>0$, such that for any $\varphi \in \mathcal{B}_{T_{p} M}^{r, \delta}, t \in[0, T]$,

$$
\mathbb{E}\left[\left\langle: \Pi(\Xi \mathcal{I}(\Xi))_{x}^{t}:, \varphi_{p}^{\lambda}\right\rangle^{2}\right] \leq C \int_{M} d(p, q)^{2-\delta}\left|\varphi_{p}^{\lambda}(q)\right|^{2} d q
$$

Hence for any $\lambda \in(0,1]$ and $\varphi \in \mathcal{B}_{T_{p} M}^{2, \delta}$,

$$
\mathbb{E}\left[\left\langle: Z_{p}^{t}:, \varphi_{p}^{\lambda}\right\rangle^{2}\right] \leq C \lambda^{-\delta}
$$

Lemma 54 For any $v>\eta>0, T \geq 0$, there exists $C>0$, such that for any $q \in M, t \in[0, T]$,

$$
\left|k_{2, t}(q, q)+k_{2, t}(p, p)-2 k_{2, t}(q, p)\right| \leq C t^{\eta} d(p, q)^{2-2 v} .
$$

Proof On the one hand, according to (18) and Theorem 43, the left-hand-side of (22) is uniformly bounded by $C_{T} t$, for all $t \in[0, T]$, for some $C_{T}>0$. On the other hand, the estimate (22) would hold true, with $\eta=0$, if $K_{t}$ would be replaced by a $C^{2}$ symmetric function on $M^{2}$. Indeed if $K: M^{2} \rightarrow \mathbb{R}$ is a $C^{2}$ symmetric function,

$$
|K(q, q)+K(p, p)-2 K(q, p)| \leq \int_{0 \leq r, s \leq 1}\left\|\nabla_{2, \dot{\gamma}_{s}} \nabla_{1, \dot{\gamma}_{r}} K\left(\gamma_{s}, \gamma_{r}\right)\right\|_{\infty} d r d s,
$$

where the index below the connexion symbol indicates the variable on which the latter is acting, and $\gamma$ is a geodesic from $p$ to $q$. According to Theorem 43, one can therefore consider $K_{t}^{2, N}=\int_{0}^{t} s P_{s}^{N} d s$ in place of $K_{t}^{2}$, as soon as $N$ is large enough. This same theorem ensures that there exists a smooth function $\Phi:[0, T] \times M^{2} \rightarrow \mathbb{R}_{\geq 0}$ such that for all $\tau \in(0, T], p, q \in M$,

$$
\mathrm{p}_{\tau}^{N}(p, q)=(2 \pi \tau)^{-1} e^{-\frac{d(p, q)^{2}}{2 \tau}} \Phi(\tau, p, q)
$$

Let us set $q_{\tau}(r)=\frac{1}{2 \pi \tau} e^{-\frac{r^{2}}{\tau}}$, for any $r, \tau>0$. We shall apply (23) to $K_{t, \varepsilon}=$ $\int_{\varepsilon}^{t} s P_{s}^{N} d s$, for any fixed $\varepsilon>0$. Up to a constant, the integrand of the right-handside of (23) is bounded by $\int_{\varepsilon}^{t}\left(d(p, q)\left\|\nabla_{1, \dot{\gamma}_{s}} q_{\tau} \circ d\left(\gamma_{s}, \gamma_{r}\right)\right\|+\| \nabla_{1, \dot{\gamma}_{s}} \nabla_{2, \dot{\gamma}_{r}} q_{\tau} \circ d\right.$ $\left.\left(\gamma_{s}, \gamma_{r}\right) \|\right) d \tau$. Let us set $R=d(p, q)$. The first term can be bounded by

$$
\begin{aligned}
R^{2} \int_{\varepsilon}^{t} \tau^{-1}|s-r| e^{-\frac{R^{2}|s-r|^{2}}{2 \tau}} d \tau & \leq R^{2}|s-r| \int_{\frac{R^{2}}{t}|r-s|^{2}}^{\frac{R^{2}}{\varepsilon}|r-s|^{2}} e^{-u / 2} \frac{d u}{u} \\
& \leq C_{T} R^{2}|s-r| \log \frac{T}{|s-r| R^{2}}
\end{aligned}
$$


and the second by

$$
\begin{aligned}
R^{2} \int_{\varepsilon}^{t}\left(\tau^{-1}+\frac{R^{2}(r-s)^{2}}{\tau^{2}}\right) e^{-\frac{R^{2}(r-s)^{2}}{2 \tau}} d \tau & \leq R^{2}\left(2 \log \frac{T}{|s-r| R^{2}}+\int_{R^{2}(r-s)^{2} / t}^{\infty} e^{-u / 2} d u\right), \\
& \leq C_{T} R^{2} \log \frac{T}{|s-r| R^{2}},
\end{aligned}
$$

for some constant $C_{T}>0$. These two bounds, once integrated in (23), imply that for any $\alpha>0$, the left-hand-side of (22) is bounded by $C_{T} d(p, q)^{2-\alpha}$, uniformly on $p, q \in M$ and $t \in[0, T]$. Using the bound $\min \{a, b\} \leq a^{\eta} b^{1-\eta}$, for $a, b, \eta \in(0,1)$, gives (22).

\section{Appendix - Higher order "polynomials"}

We recall the regularity structure of polynomial functions in flat space $\mathbb{R}^{d}$ given in [9]. It is used to abstractly describe functions in $C^{\gamma}\left(\mathbb{R}^{d}\right), \gamma>0$, and also forms a central ingredient for general regularity structures associated with singular SPDEs. Let $\gamma>0$ and $n=\lfloor\gamma\rfloor$, that is $n \in \mathbb{N}_{0}$ and $\gamma \in(n, n+1]$. For simplicity of notation let $d=1$. Define

$$
\mathcal{T}^{\text {flat }}:=\bigoplus_{\ell=0, \ldots, n} \operatorname{span}\left\{X^{\lrcorner}\right\}
$$

where $\operatorname{span}\left\{X^{\ell}\right\}$ denotes the one-dimensional vector space spanned by the abstract symbol $X^{\ell}$. Hence $\mathcal{T}_{\text {flat }} \simeq \mathbb{R}^{n+1}$.

Given $x, y \in \mathbb{R}$ and $\ell \in \mathbb{N}_{0}$, we define the linear maps, $\Pi_{x}: \mathcal{T}^{\text {flat }} \rightarrow \subset \mathcal{D}^{\prime}(\mathbb{R})$ and $\Gamma_{x \leftarrow y}: \mathcal{T}^{\text {flat }} \rightarrow \mathcal{T}^{\text {flat }}$, which are uniquely determined by

$$
\begin{aligned}
& \Pi_{x}^{\text {flat }} X^{\sqsupset}:=(\cdot-x)^{\ell} \\
& \Gamma_{x \leftarrow y}^{\text {flat }} X^{\exists}:=\sum_{i \leq \ell}\left(\begin{array}{l}
\ell \\
i
\end{array}\right)(x-y)^{\ell-i} X^{i} .
\end{aligned}
$$

In this case one has $\Pi_{x}^{\text {flat }} \Gamma_{x \leftarrow y}^{\text {flat }} \tau=\Pi_{y}^{\text {flat }} \tau$ for all $\tau \in \mathcal{T}_{\text {flat }}$. One can use this regularity structure to describe regular functions.

Lemma 55 ([9, Lemma 2.12]) Let $f: \mathbb{R} \rightarrow \mathbb{R}$. Then $f \in C^{\gamma}(\mathbb{R})$ if and only if there exists $\hat{f}: \mathbb{R} \rightarrow \mathcal{T}^{\text {flat }}$ with $\hat{f}_{0}(x)=f(x)$ and

$$
\left\|\hat{f}(x)-\Gamma_{x \leftarrow y}^{\text {flat }} \hat{f}(y)\right\|_{\ell} \lesssim|x-y|^{\gamma-\ell} .
$$

In that case $\hat{f}_{\ell}(x)=f^{(\ell)}(x), \ell=0, \ldots, n .^{11}$

11 Here we recall the notation of $\hat{f}_{\ell}(x)$ as the component of $\hat{f}(x)$ on the $\ell$-th homogeneity, i.e. the coefficient in front of $\boldsymbol{X}^{\ell}$. 


\subsection{Higher order covariant derivatives}

We want to mirror as best we can the flat space polynomial model described above, in the general context of a closed $d$ dimensional Riemannian manifold. In order to do to this we need to store higher order derivatives of functions $f: M \rightarrow \mathbb{R}$ in a coordinate independent fashion. There is a canonical way to do this on a Riemannian manifold by making use of the associated Levi-Civita connection.

We recall the notion of higher order covariant derivatives of functions $f: M \rightarrow \mathbb{R}$ on a Riemannian manifold with Levi-Civita ${ }^{12}$ connection $\nabla$ (see for example [14, Lemma 4.6]).

Definition 56 Define $\left.\nabla^{\ell}\right|_{p} f \in\left[T_{p}^{*} M\right]^{\otimes \ell} \cong\left[T_{p} M^{\otimes \ell}\right]^{*}$ by,

$$
\left.\nabla^{0}\right|_{p} f=f(p),\left\langle\left.\nabla\right|_{p} f, X_{1}(p)\right\rangle=\left\langle\left. d\right|_{p} f, X_{1}(p)\right\rangle
$$

and then inductively by;

$$
\begin{aligned}
& \left\langle\left.\nabla^{\ell}\right|_{p} f, X_{1}(p) \otimes \cdots \otimes X_{\ell}(p)\right\rangle \\
& =\left.\left[X_{1}\left\langle\nabla^{\ell-1} f, X_{2} \otimes \cdots \otimes X_{\ell}\right\rangle\right]\right|_{p} \\
& \quad-\left.\sum_{m=2}^{\ell}\left\langle\nabla^{\ell-1} f, X_{2} \otimes \cdots \otimes X_{m-1} \otimes \nabla_{X_{1}} X_{m} \otimes X_{m+1} \otimes \cdots \otimes X_{\ell}\right\rangle\right|_{p},
\end{aligned}
$$

where $X_{1}, \ldots, X_{\ell}$ are arbitrary vector fields on $M$.

A few remarks are in order.

1. As the notation suggests, $\left.\nabla^{\ell}\right|_{p} f$ is indeed tensorial, i.e. the right side of the previously displayed equation really only depends on the vector fields, $\left\{X_{i}\right\}_{i=1}^{\ell}$, through their values at $p$.

2. In the literature $\nabla f$ sometimes denotes the gradient of $f$. We never use the gradient of a function in this work.

3. We shall also sometimes write $\nabla_{W}^{\ell} f=\left\langle\left.\nabla^{\ell}\right|_{p} f, W\right\rangle$ for any $W \in\left(T_{p} M\right)^{\otimes \ell}$.

For a curve $\gamma$ in $M$ we will write

$$
/ /{ }_{t}\left(\gamma_{v}\right): T_{\gamma(0)} M \rightarrow T_{\gamma(t)}
$$

for parallel translation along $\gamma$. For a tensor field $W$ along $\gamma$ we write

$$
\frac{\nabla}{d t}[W]
$$

for the covariant derivative of $W$ along $\gamma$.

12 In general, $\nabla$ can be any affine connection. 
Lemma 57 If $f$ is an $\ell$-times continuously differentiable function in a neighborhood of $p \in M, v \in T_{p} M$, and $\gamma_{v}(t):=\exp _{p}(t v)$, then

$$
\left.\frac{d^{\ell}}{d t^{\ell}}\right|_{t=0} f\left(\gamma_{v}(t)\right)=\nabla_{v^{\otimes \ell}}^{\ell} f, \text { for all } v \in T_{p} M .
$$

More generally, if $\ell, n \in \mathbb{N}_{0}, f$ is an $(\ell+n+1)$-times continuously differentiable function in a neighborhood of $p \in M$ and $W_{t}:=/ /{ }_{t}\left(\gamma_{v}\right)^{\otimes \ell} W_{0}$, then

$$
\frac{d^{k}}{d t^{k}} \nabla_{W_{t}}^{\ell} f=\nabla_{\dot{\gamma}_{v}(t)^{\otimes k} \otimes W_{t}}^{\ell+k} f \forall 0 \leq k \leq n+1 .
$$

Proof Let $\gamma_{v}(t):=\exp _{p}(t v)$ so that $\gamma_{v}(t)$ solves the geodesic differential equation, $\nabla \dot{\gamma}_{v}(t) / d t=0$ with $\dot{\gamma}_{v}(0)=v$. The proof is completed by showing (by induction) that

$$
\frac{d^{k}}{d t^{k}} f\left(\gamma_{v}(t)\right)=\nabla_{\dot{\gamma}_{v}(t)^{\otimes k}}^{k} f \text { for } 1 \leq k \leq \ell .
$$

The case $k=1$ amounts to the definition that $\nabla_{v} f=v f=d f(v)$ for all $v \in T M$. For the induction step we have by the product rule;

$$
\begin{aligned}
\frac{d^{k+1}}{d t^{k+1}} f\left(\gamma_{v}(t)\right) & =\frac{d}{d t} \nabla_{\dot{\gamma}_{v}(t)^{\otimes k}}^{k} f=\nabla_{\dot{\gamma}_{v}(t)^{\otimes(k+1)}}^{k+1} f+\nabla_{\frac{\nabla}{d t}\left[\dot{\gamma}_{v}(t)^{\otimes k}\right]}^{k} f \\
& =\nabla_{\dot{\gamma}_{v}(t)^{\otimes(k+1)}}^{k+1} f,
\end{aligned}
$$

wherein the last equality we have again used the product rule to conclude that $\frac{\nabla}{d t}\left[\dot{\gamma}_{v}(t)^{\otimes k}\right]=0$. The result now follows by evaluating (27) at $k=\ell$ and $t=0$. The more general assertion in (26) is proved similarly. One only need to observe that $\frac{\nabla}{d t} W_{t}=0$, by definition of parallel transportation, and hence the presence of $W_{t}$ in the expressions in no way changes the computations.

Definition 58 (Symmetrizations) If $V$ is a real vector space and $\ell \in \mathbb{N}$, we let $\operatorname{Sym}_{\ell}$ : $V^{\otimes \ell} \rightarrow V^{\otimes \ell}$ denote the symmetrization projection uniquely determined by

$$
\operatorname{Sym}_{\ell}\left(v_{1} \otimes \cdots \otimes v_{\ell}\right)=\frac{1}{\ell !} \sum_{\sigma \in S_{\ell}} v_{\sigma(1)} \otimes \cdots \otimes v_{\sigma(\ell)}
$$

where $S_{\ell}$ is the permutation group on $\{1,2, \ldots, \ell\}$. Often we will simply write Sym for $\operatorname{Sym}_{\ell}$ as it will typically be clear what $\ell$ is from the argument put into the symmetrization function.

As usual we let $V^{*}$ denote the dual space to a vector space $V$ and let $\langle\cdot, \cdot\rangle$ denote the pairing between a vector space and its dual. We will often identify $\left(V^{*}\right)^{\otimes \ell}$ with $\left[V^{\otimes \ell}\right]^{*}$ where the identification is uniquely determined by

$\left\langle\varepsilon^{1} \otimes \ldots \cdots \otimes \varepsilon^{\ell}, v_{1} \otimes \cdots \otimes v_{\ell}\right\rangle=\varepsilon^{1}\left(v_{1}\right) \cdots \varepsilon^{\ell}\left(v_{\ell}\right) \forall\left\{\varepsilon^{i}\right\}_{i=1}^{\ell} \subset V^{*}$ and $\left\{v_{i}\right\}_{i=1}^{\ell} \subset V$. 
We also identify $\left(V^{*}\right)^{\otimes \ell}$ with the space of multi-linear maps from $V^{\ell} \rightarrow \mathbb{R}$ using,

$$
T\left(v_{1}, \ldots, v_{\ell}\right)=\left\langle T, v_{1} \otimes \cdots \otimes v_{\ell}\right\rangle \forall T \in\left(V^{*}\right)^{\otimes \ell} \text { and }\left\{v_{i}\right\}_{i=1}^{\ell} \subset V .
$$

Under these identification we have

$$
\langle\operatorname{Sym}[T], W\rangle=\langle T, \operatorname{Sym}[W]\rangle \forall T \in\left(V^{*}\right)^{\otimes \ell} \text { and } W \in V^{\otimes \ell} .
$$

Remark 59 If $T \in\left[V^{*}\right]^{\otimes \ell}$ and $v_{1}, \ldots, v_{\ell} \in V$, then

$$
\left.\frac{\partial}{\partial s_{1}} \ldots \frac{\partial}{\partial s_{\ell}}\right|_{s_{1}=\cdots=s_{\ell}=0} T\left(\left(s_{1} v_{1}+\cdots+s_{\ell} v_{\ell}\right)^{\otimes \ell}\right)=\sum_{\sigma \in S_{\ell}} T\left(v_{\sigma(1)}, \ldots, v_{\sigma(\ell)}\right)
$$

and therefore,

$$
\operatorname{Sym}[T]\left(v_{1}, \ldots, v_{\ell}\right):=\left.\frac{1}{\ell !} \frac{\partial}{\partial s_{1}} \ldots \frac{\partial}{\partial s_{\ell}}\right|_{s_{1}=\cdots=s_{\ell}=0} T\left(\left(s_{1} v_{1}+\cdots+s_{\ell} v_{\ell}\right)^{\otimes \ell}\right)
$$

This formula shows that the symmetric part Sym $[T]$ of $T$ is completely determined by the knowledge of $T(v, v, \ldots, v)$ for all $v \in V$.

Definition 60 Let $\Sigma^{\ell} T_{p}^{*} M$ denote the symmetric tensors in $\left[T_{p}^{*} M\right]^{\otimes \ell}$ and for $T \in$ $\left[T_{p}^{*} M\right]^{\otimes \ell}$, let $\operatorname{Sym}[T] \in \Sigma^{\ell} T_{p}^{*} M$ denote the symmetrization of $T$ as above.

Example 61 If $U$ is an open subset of $M$ and $f$ is $\ell$-times continuously differentiable on $U$, then $\operatorname{Sym}\left[\nabla^{\ell} f\right]$ defines a local section (over $U$ ) of $\Sigma^{\ell} T^{*} M$. Moreover since $v^{\otimes \ell}$ is symmetric for all $v \in T_{p} M$ we may write (25) as

$$
\left.\frac{d^{\ell}}{d t^{\ell}}\right|_{t=0} f\left(\gamma_{v}(t)\right)=\left\langle\operatorname{Sym}\left[\nabla_{p}^{\ell} f\right], v^{\otimes \ell}\right\rangle \text {, for all } v \in T_{p} M
$$

Theorem 62 (Taylor's Theorem on $M$ ) Let $\ell, n \in \mathbb{N}_{0}, p \in M, v \in T_{p} M, \gamma_{v}(t):=$ $\exp _{p}(t v), / /_{t}\left(\gamma_{v}\right): T_{\gamma_{v}(0)} M \rightarrow T_{\gamma_{v}(t)} M, W_{0} \in T_{p} M^{\otimes \ell}$, and $W_{t}:=/ /_{t}\left(\gamma_{v}\right)^{\otimes \ell} W_{0}$. If $f$ is $(\ell+n+1)$-times continuously differentiable on $U$, where $U$ is an open set containing $\gamma_{v}([0,1])$, then

$$
\nabla_{W_{1}}^{\ell} f=\sum_{k=0}^{n} \frac{1}{k !} \nabla_{v^{\otimes k} \otimes W_{0}}^{\ell+k} f+\frac{1}{n !} \int_{0}^{1}\left[\nabla_{\dot{\gamma}_{v}(t)^{\otimes(n+1)} \otimes W_{t}}^{\ell+n+1} f\right] \cdot(1-t)^{n} d t .
$$

When $\ell=0$ the previous equation reads as (also see [5, Theorem 6.1])

$$
f\left(\exp _{p}(v)\right)=\sum_{k=0}^{n} \frac{1}{k !} \nabla_{v^{\otimes k}}^{k} f+\frac{1}{n !} \int_{0}^{1}\left[\nabla_{\dot{\gamma}_{v}(t)^{\otimes(n+1)}}^{n+1} f\right] \cdot(1-t)^{n} d t
$$




$$
\begin{aligned}
= & \sum_{k=0}^{n} \frac{1}{k !}\left\langle\operatorname{Sym}\left[\nabla_{p}^{k} f\right], v^{\otimes k}\right\rangle \\
& +\frac{1}{n !} \int_{0}^{1}\left\langle\operatorname{Sym}\left[\nabla_{\gamma_{v}(t)}^{n+1} f\right], \dot{\gamma}_{v}(t)^{\otimes(n+1)}\right\rangle(1-t)^{n} d t,
\end{aligned}
$$

where

$$
\frac{1}{0 !}\left\langle\left.\operatorname{Sym} \nabla^{0}\right|_{p} f, v^{\otimes 0}\right\rangle:=f(p) .
$$

Proof Let $g(t):=\nabla_{W_{t}}^{\ell} f$ and recall that the standard Taylor's theorem with remainder states;

$$
g(1)=\sum_{k=0}^{n} \frac{1}{n !} g^{(k)}(0)+\frac{1}{n !} \int_{0}^{1} g^{(n+1)}(t)(1-t)^{n} d t .
$$

The results now follow by using Lemma 57 in order to compute the $g^{(k)}(t)$ for $1 \leq k \leq n+1$.

Remark 63 Since parallel translation is isometric it follows (continuing the notation in Theorem 62) that

$$
\left|\nabla_{\dot{\gamma}_{v}(t)^{\otimes(n+1)} \otimes W_{t}}^{\ell+n+1} f\right| \leq\left\|\nabla^{\ell+n+1} f\right\|_{\left[T_{\gamma_{v}(t)}^{*} M\right]^{\otimes(\ell+n+1)}\|v\|^{n+1}\left\|W_{0}\right\|}
$$

and hence

$$
\begin{aligned}
& \left|\nabla_{W_{1}}^{\ell} f-\sum_{k=0}^{n} \frac{1}{k !} \nabla_{v^{\otimes k} \otimes W_{0}}^{\ell+k} f\right| \\
& \leq \frac{1}{(n+1) !}\left\|W_{0}\right\| \cdot \max _{0 \leq t \leq 1}\left\|\nabla^{\ell+n+1} f\right\|_{\left[T_{\gamma_{v}(t)}^{*} M\right]^{\otimes(\ell+n+1)} \cdot d\left(p, \exp _{p}(v)\right)^{n+1}} .
\end{aligned}
$$

Since $M$ is a compact Riemannian manifold it is necessarily complete and therefore, by the Hopf-Rinow theorem, for each $q \in M$ we may find at least one $v \in T_{p} M$ such that $q=\exp _{p}(v)$ and $d(q, p)=|v|$. Using these remarks we can reformulate (30) as follows.

Corollary 64 If $f$ is $(n+1)$-times continuously differentiable on $M, p, q \in M$, and $v \in T_{p} M$ is chosen so that $q=\exp _{p}(v)$ and $d(q, p)=|v|$, then

$$
f(q)=\sum_{k=0}^{n} \frac{1}{k !}\left\langle\left.\operatorname{Sym} \nabla^{k}\right|_{p} f, v^{\otimes k}\right\rangle+O_{f}\left(d(p, q)^{n+1}\right)
$$


where

$$
\left|O_{f}\left(d(p, q)^{n+1}\right)\right| \leq \frac{1}{(n+1) !} \max _{m \in M}\left\|\operatorname{Sym}\left[\left.\nabla^{n+1}\right|_{m} f\right]\right\| d(p, q)^{n+1} .
$$

Furthermore if $f$ is n-times continuously differentiable on $M$ then

$$
f(q)=\sum_{k=0}^{n} \frac{1}{k !}\left\langle\left.\operatorname{Sym} \nabla^{k}\right|_{p} f, v^{\otimes k}\right\rangle+o_{f}\left(d(p, q)^{n}\right) .
$$

Definition 65 (Taylor approximations) Suppose that $U \subset M$ is an open subset of $M$, $p \in U, f$ a $n$-times continuously differentiable function on $M$ and $\varepsilon>0$ is sufficiently small so that $B_{M}(p, \varepsilon) \subset U$ and $\varepsilon$ is smaller than the injectivity radius of $M$. We then define, Tay $_{p}^{n} f \in C^{\infty}\left(B_{M}(p, \varepsilon)\right)$ by

$$
\left(\operatorname{Tay}_{p}^{n} f\right)(q):=\sum_{k=0}^{n} \frac{1}{k !}\left\langle\left.\operatorname{Sym}^{k}\right|_{p} f,\left[\exp _{p}^{-1}(q)\right]^{\otimes k}\right\rangle .
$$

Remark 66 With this notation, Corollary 64 reads as

$$
f(q)=\left(\operatorname{Tay}_{p}^{n} f\right)(q)+o_{f}\left(d(p, q)^{n}\right) .
$$

In the case $M=\mathbb{R}^{d}$ and $f$ is a polynomial of degree at most $n$, it follows by Taylor's theorem that $f=\operatorname{Tay}_{p}^{n} f$ for all $p \in \mathbb{R}^{d}$. So in the flat case the error term here is no longer present.

Lemma 67 If $f$ is a n-times continuously differentiable function on $M$ and $f(q)=$ $o\left(d(p, q)^{n}\right)$, then $(V f)(p)=0$ for any $n^{\text {th }}$ - order differential operator $V$ and in particular, $\left.\nabla^{k}\right|_{p} f=0$ for all $0 \leq k \leq n$.

Proof Let $(\Psi, U)$ be a chart on with $p \in U$ and $\Psi(p)=0$ and define $F:=f \circ \Psi^{-1} \in$ $C^{n}(\tilde{U}:=\Psi(U))$. Then the give assumption implies $F(x)=o\left(|x|^{n}\right)$ and therefore for any $x \in \mathbb{R}^{d}$ and $t \in \mathbb{R}$ small we have $F(t x)=o\left(t^{n}\right)$ from which it easily follows that

$$
0=\left.\frac{d^{k}}{d t^{k}} F(t x)\right|_{t=0}=\left\langle\left(D^{k} F\right)(0), x^{\otimes k}\right\rangle \text { for all } 0 \leq k \leq n
$$

As $\left(D^{k} F\right)(0)$ is symmetric and $x \in \mathbb{R}^{d}$ was arbitrary we may conclude that $\left(D^{k} F\right)(0)=\mathbf{0} \in\left(\mathbb{R}^{d}\right)^{* \otimes k}$ for $0 \leq k \leq n$. As any $n^{\text {th }}$ - order differential operator $U$ on $C^{n}(M)$ may be written locally as

$$
V f=\sum_{k=0}^{n}\left\langle\left(D^{k} F\right)(\Psi), W_{k}\right\rangle
$$


for some smooth functions, $W_{k}: U \rightarrow\left(\mathbb{R}^{d}\right)^{\otimes k}$ for each $0 \leq k \leq n$, it follows that

$$
(V f)(p)=\sum_{k=0}^{n}\left\langle\left(D^{k} F\right)(\Psi(p)), W_{k}(p)\right\rangle=\sum_{k=0}^{n}\left\langle\left(D^{k} F\right)(0), W_{k}(p)\right\rangle=0
$$

Corollary 68 If $f$ a n-times continuously differentiable function on $M$ and $V$ is an $n^{\text {th }}$ - order differential operator, then

$$
(V f)(p)=\left[V\left(\operatorname{Tay}_{p}^{n} f\right)\right](p)
$$

and in particular,

$$
\left.\nabla^{n}\right|_{p} f=\left[\left.\nabla^{n}\right|_{p}\left(\operatorname{Tay}_{p}^{n} f\right)\right](p)
$$

from which it follows that $\left.\nabla^{n}\right|_{p} f$ is a linear combination of $\left\{\left.\operatorname{Sym} \nabla^{k}\right|_{p} f\right\}_{k=0}^{n}$.

We will make the last assertion of Corollary 68 more explicitly in Corollary 74 and Remark 76. The upshot is that there is no loss of information in only keeping track of the symmetrizations of the covariant derivatives.

Corollary 69 If $f \in C^{\infty}(M), p, q \in M$ with $d(p, q)$ then for $0 \leq k \leq n$ we have

$$
\left\|\left.\nabla^{k}\right|_{q}\left[f-\left(\operatorname{Tay}_{p}^{n} f\right)\right]\right\| \leq \frac{1}{(n+1-k) !} \max _{0 \leq t \leq 1}\left\|\left.\nabla^{n+1}\right|_{\gamma_{v}(t)}\left(f-\operatorname{Tay}_{p}^{n} f\right)\right\| \cdot d(p, q)^{n+1-k},
$$

where $v:=\exp _{p}^{-1}(q)$.

Proof Let us apply the estimate in (31) with $f$ replaced by $g:=f-\operatorname{Tay}_{p}^{n} f$ keeping in mind that $\left.\nabla^{k}\right|_{p}\left[f-\left(\operatorname{Tay}_{p}^{n} f\right)\right]=0$ for $0 \leq k \leq n$ by Corollary 68. This allows us to conclude for $W_{0} \in T_{p} M^{\otimes k}$ that

$\left|\nabla_{W_{1}}^{k}\left[f-\operatorname{Tay}_{p}^{n} f\right]\right| \leq \frac{1}{(n+1-k) !}\left\|W_{0}\right\| \cdot \max _{0 \leq t \leq 1}\left\|\nabla^{n+1} g\right\|_{\left[T_{\gamma_{v}(t)}^{*} M\right]^{\otimes(n+1)} \cdot d(p, q)^{n+1-k}}$

where $v:=\exp _{p}^{-1}(q)$. As the map $W_{0} \rightarrow W_{1}$ is an isometry it follows that

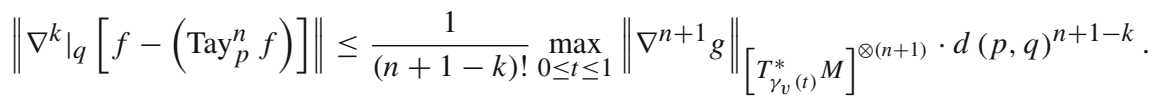




\subsubsection{Symmetric parts of covariant derivatives determine all derivatives}

We will now make Corollary 68 more precise.

Definition 70 If $(x, U:=\operatorname{dom}(x))$ is a chart on $M$, let $D^{x}$ denote the flat covariant derivative on $T U$ determined by $D^{x} \frac{\partial}{\partial x^{j}}=0$ for $1 \leq j \leq d$.

Remark 71 If $V=\sum_{j=1}^{d} V_{j} \frac{\partial}{\partial x^{j}}$ is a vector field on $U$ and $v \in T_{m} M$ then $D_{v}^{x} V=$ $\left.\sum_{j=1}^{d}\left(v V_{j}\right) \frac{\partial}{\partial x^{j}}\right|_{m}$. Using $D^{x} \frac{\partial}{\partial x^{j}}=0$, it easily follows that for all $\ell \in \mathbb{N}$ and any $\ell$-times continuously differentiable function $f$ we have

$$
\left\langle\left.\left(D^{x}\right)^{\ell}\right|_{m} f, \frac{\partial}{\partial x^{i_{1}}} \otimes \cdots \otimes \frac{\partial}{\partial x^{i_{\ell}}}\right\rangle=\left.\frac{\partial}{\partial x^{i_{1}}} \cdots \frac{\partial}{\partial x^{i_{\ell}}}\right|_{m} f
$$

and in particular $\left(D^{x}\right)^{\ell} f \in \Sigma^{\ell} T^{*} U$.

Lemma 72 Suppose that $(x, U:=\operatorname{dom}(x))$ is a chart on $M, D=D^{x}$ is the flat covariant derivative of Definition 70. Then, there exists a family of sections $Q_{\ell, n} \in$ $\Gamma\left[\operatorname{Hom}\left[T U^{\otimes n}, T U^{\otimes \ell}\right]\right]$ for $1 \leq \ell \leq n$, such that $Q_{n, n}=\mathrm{id}$ and for all $n$-times continuously differentiable functions $f$,

$$
\left\langle\left.\nabla^{n}\right|_{p} f, W\right\rangle=\sum_{\ell=1}^{n}\left\langle\left. D^{\ell}\right|_{p} f, Q_{\ell, n} W\right\rangle \forall W \in\left[T_{p} M\right]^{\otimes n}
$$

Proof Let $D=D^{x}$ and $\Gamma$ be the End $(T U)$ - valued connection one form on $T U$ so that $\nabla=D+\Gamma$. It is enough to verify that (32) holds on a basis for $T_{p} U^{\otimes n}$. To this end, let $i_{j} \in\{1,2 \ldots, d\}$, for $1 \leq j \leq n$ and let $V_{j}=\frac{\partial}{\partial x^{i} j}$. Then,

$$
\left\langle\nabla f, V_{1}\right\rangle=V_{1} f=\left\langle D f, V_{1}\right\rangle
$$

which shows that (32) holds for $n=1$. For the sake of completing the proof by induction, let us now assume that (32) holds at level $n-1$ and below. In particular we assume

$$
\left\langle\nabla^{n-1} f, V_{n-1} \otimes \cdots \otimes V_{1}\right\rangle=\sum_{\ell=1}^{n-1}\left\langle D^{\ell} f, Q_{\ell, n-1} V_{n-1} \otimes \cdots \otimes V_{1}\right\rangle
$$

On one hand,

$$
\begin{aligned}
& V_{n}\left\langle\nabla^{n-1} f, V_{n-1} \otimes \cdots \otimes V_{1}\right\rangle \\
& \quad=\left\langle\nabla^{n} f, V_{n} \otimes \cdots \otimes V_{1}\right\rangle+\left\langle\nabla^{n-1} f, \nabla_{V_{n}}\left[V_{n-1} \otimes \cdots \otimes V_{1}\right]\right\rangle \\
& \quad=\left\langle\nabla^{n} f, V_{n} \otimes \cdots \otimes V_{1}\right\rangle
\end{aligned}
$$




$$
+\sum_{k=1}^{n-1}\left\langle\nabla^{n-1} f, V_{n-1} \otimes \cdots \otimes\left[\Gamma\left(V_{n}\right) V_{k}\right] \otimes \cdots \otimes V_{1}\right\rangle,
$$

while on the other hand (using the induction hypothesis, the product rule, and $D V_{k}=0$ for all $k$ ),

$$
\begin{aligned}
V_{n}\left\langle\nabla^{n-1} f, V_{n-1} \otimes \cdots \otimes V_{1}\right\rangle= & \sum_{\ell=1}^{n-1} V_{n}\left\langle D^{\ell} f, Q_{\ell, n-1} V_{n-1} \otimes \cdots \otimes V_{1}\right\rangle \\
= & \sum_{\ell=1}^{n-1}\left\langle D^{\ell+1} f, V_{n} \otimes Q_{\ell, n-1} V_{n-1} \otimes \cdots \otimes V_{1}\right\rangle \\
& +\sum_{\ell=1}^{n-1}\left\langle D^{\ell} f,\left(D_{V_{n}} Q_{\ell, n-1}\right) V_{n-1} \otimes \cdots \otimes V_{1}\right\rangle \\
= & \left\langle D^{n} f, V_{n} \otimes \cdots \otimes V_{1}\right\rangle \\
& +\sum_{\ell=1}^{n-2}\left\langle D^{\ell} f, V_{n} \otimes Q_{\ell, n-1} V_{n-1} \otimes \cdots \otimes V_{1}\right\rangle \\
& +\sum_{\ell=1}^{n-1}\left\langle D^{\ell} f,\left(D_{V_{n}} Q_{\ell, n-1}\right) V_{n-1} \otimes \cdots \otimes V_{1}\right\rangle .
\end{aligned}
$$

Comparing the last two displayed equations shows,

$$
\begin{aligned}
& \nabla_{V_{n} \otimes \cdots \otimes V_{1}}^{n} f=\left\langle D^{n} f, V_{n} \otimes \cdots \otimes V_{1}\right\rangle+\left\langle D f,\left[D_{V_{n}} Q_{1, n-1}\right] V_{n-1} \otimes \cdots \otimes V_{1}\right\rangle \\
& \quad+\sum_{\ell=2}^{n-1}\left\langle D^{\ell} f, V_{n} \otimes Q_{\ell-1, n-1} \cdot V_{n-1} \otimes \cdots \otimes V_{1}+\left[D_{V_{n}} Q_{\ell, n-1}\right] V_{n-1} \otimes \cdots \otimes V_{1}\right\rangle \\
& \quad-\sum_{k=1}^{n-1}\left\langle\nabla^{n-1} f, V_{n-1} \otimes \cdots \otimes \Gamma\left(V_{n}\right) V_{k} \otimes \cdots \otimes V_{1}\right\rangle .
\end{aligned}
$$

From this expression it follows that $\nabla_{V_{n} \otimes \cdots \otimes V_{1}}^{n} f$ may be expressed in the form claimed in (32).

Corollary 73 Let us continue the notation in Lemma 72. Then, there exists

$$
\bar{Q}_{\ell, n} \in \Gamma\left[\operatorname{Hom}\left[T U^{\otimes n}, T U^{\otimes \ell}\right]\right], \text { for } 1 \leq \ell \leq n
$$

such that $\bar{Q}_{n, n}=$ id and for all n-times continuously differentiable functions $f$,

$$
\left\langle D^{n} f, W\right\rangle=\sum_{\ell=1}^{n}\left\langle\operatorname{Sym}\left[\nabla^{\ell} f\right], \bar{Q}_{\ell, n} W\right\rangle, \forall W \in T M^{\otimes n}
$$


Proof The proof is again by induction on $n$. For $n=1$, we have $D_{W} f=W f=\nabla_{W} f$, so there is nothing to prove. For the inductive step, suppose that (33) holds at level $n-1$ and below. From (32) with $W$ replaced by $\operatorname{Sym}_{n} W$, it follows that,

$$
\begin{aligned}
\left\langle\operatorname{Sym}\left[\nabla^{n} f\right], W\right\rangle & =\left\langle\nabla^{n} f, \operatorname{Sym}_{n} W\right\rangle=\sum_{\ell=1}^{n}\left\langle D^{\ell} f, Q_{\ell, n} \operatorname{Sym}_{n} W\right\rangle \\
& =\left\langle D^{n} f, W\right\rangle+\sum_{\ell=1}^{n-1}\left\langle D^{\ell} f, Q_{\ell, n} \operatorname{Sym}_{n} W\right\rangle
\end{aligned}
$$

wherein the last equality we have used that $D^{n} f$ is already symmetric. From the previous equation along with the inductive hypothesis, we conclude that $\left\langle D^{n} f, W\right\rangle$ may be expressed as described in (33).

Corollary 74 If $\nabla$ is a covariant derivative on $T M$, then there exists

$$
Q_{\ell, n}^{\nabla} \in \Gamma\left[\operatorname{Hom}\left[T M^{\otimes n}, T M^{\otimes \ell}\right]\right], \text { for } 1 \leq \ell \leq n
$$

such that $Q_{n, n}^{\nabla}=\mathrm{id}$ and for all $n$-times continuously differentiable functions $f$,

$$
\left\langle\nabla^{n} f, W\right\rangle=\sum_{\ell=1}^{n}\left\langle\operatorname{Sym} \nabla^{\ell} f, Q_{\ell, n}^{\nabla} W\right\rangle, \forall W \in T M^{\otimes n}
$$

Proof First suppose that $M=U$, as in Lemma 72. Then combining the results of Lemma 72 and Corollary 73, there exists $Q_{\ell, n}^{x} \in \Gamma\left[\operatorname{Hom}\left[T U^{\otimes n}, T U^{\otimes \ell}\right]\right]$ such that (34) holds for all $W \in T U^{\otimes M}$. Let $\left\{x_{\alpha}\right\}_{\alpha=1}^{N}$ be a collection of charts on $M$ such that $\left\{\operatorname{dom}\left(x_{\alpha}\right)\right\}_{\alpha=1}^{N}$ is an open cover of $M$ and $\left\{\psi_{\alpha}\right\}_{\alpha=1}^{N}$ be a partition of unity relative to this cover. To complete the proof we define

$$
Q_{\ell, n}^{\nabla}:=\sum_{\alpha=1}^{N} \psi_{\alpha} Q_{\ell, n}^{x_{\alpha}}
$$

We note the following corollary for completeness.

Corollary 75 If $\nabla$ is a covariant derivative on $T M$ and $L$ is a linear $n^{\text {th }}$ - order differential operator on $C^{\infty}(M)$, then there exists smooth sections, $W_{\ell} \in \Gamma\left(\Sigma^{\ell} T M\right)$ for $0 \leq \ell \leq n$ such that

$$
L f=\sum_{\ell=0}^{n} \nabla_{W_{\ell}}^{\ell} f \text { for all } f \in C^{\infty}(M)
$$


Proof By definition $L f$ is locally given by $L f=\sum_{\ell=0}^{n}\left\langle D^{n} f, A_{n}\right\rangle$ for some $A_{n} \in$ $\Gamma\left(\Sigma^{\ell} T U\right)$. Using Corollaries 73 and 74, we may locally express $L f$ as in (35). The global picture may then be constructed using a partition of unity argument.

Remark 76 Our Proof of Corollary 74 was local in nature and hence does not give much information about how the $Q_{\ell, n}^{\nabla}$ depend on $\nabla$. It is possible to give a global Proof of Corollary 74 which would show that $Q_{\ell, n}^{\nabla}$ may be constructed from certain combinations of covariant derivatives of the torsion and curvature tensor of $\nabla$. Here is a sketch of this argument. In this sketch we let $v \wedge w:=v \otimes w-w \otimes v$ for any $v, w \in T_{p} M$.

1. If $v_{1}, \ldots, v_{n} \in T_{p} M$ and $1 \leq i<n$, then

$$
\begin{aligned}
& \nabla_{v_{n} \otimes \cdots \otimes v_{i+2} \otimes\left[v_{i+1} \wedge v_{i}\right] \otimes v_{i-1} \otimes \cdots \otimes v_{1}}^{n} f \\
& =\left\langle\nabla_{v_{n} \otimes \cdots \otimes v_{i+2}}^{n-i-1}\left[R(\cdot, \cdot) \nabla^{i-1} f\right], v_{i+1} \otimes v_{i} \otimes v_{i-1} \otimes \cdots \otimes v_{1}\right\rangle \\
& \quad+\left\langle\nabla_{v_{n} \otimes \cdots \otimes v_{i+2}}^{n-i-1}\left[\nabla_{T(\cdot,)} \nabla^{i-1} f\right], v_{i+1} \otimes v_{i} \otimes v_{i-1} \otimes \cdots \otimes v_{1}\right\rangle
\end{aligned}
$$

where $R(\cdot, \cdot) \nabla^{i-1} f$ is the appropriate action of the curvature tensor of $\nabla$ on $\nabla^{i-1} f$ and $T$ is the torsion tensor of $\nabla$.

2. As a consequence of item 1 . and the fact that every permutation is a composition of transpositions, it follows that for any permutation $\sigma \in S_{n}$,

$$
\nabla_{v_{\sigma(n)}}^{n} \otimes \cdots \otimes v_{\sigma(1)} f=\nabla_{v_{n} \otimes \cdots \otimes v_{1}}^{n} f+\sum_{\ell=1}^{n-1}\left\langle\nabla^{\ell} f, \mathbf{Q}(\sigma)_{\ell, n} v_{n} \otimes \cdots \otimes v_{1}\right\rangle,
$$

where $\mathbf{Q}(\sigma)_{\ell, n} \in \Gamma\left[\operatorname{Hom}\left[T M^{\otimes n}, T M^{\otimes \ell}\right]\right]$ are constructed from certain combinations of covariant derivatives of the torsion and curvature tensor of $\nabla$.

3. Summing (36) on $\sigma$ and then dividing by $n$ ! and setting

$$
\mathbf{Q}_{\ell, n}=\frac{1}{n !} \sum_{\sigma \in S_{n}} \mathbf{Q}(\sigma)_{\ell, n}
$$

shows

$$
\left\langle\operatorname{Sym} \nabla^{n} f, v_{n} \otimes \cdots \otimes v_{1}\right\rangle=\left\langle\nabla^{n} f, v_{n} \otimes \cdots \otimes v_{1}\right\rangle+\sum_{\ell=1}^{n-1}\left\langle\nabla^{\ell} f, \mathbf{Q}_{\ell, n} v_{n} \otimes \cdots \otimes v_{1}\right\rangle,
$$

where the $\mathbf{Q}_{\ell, n} \in \Gamma\left[\operatorname{Hom}\left[T M^{\otimes n}, T M^{\otimes \ell}\right]\right]$ are constructed from certain combinations of covariant derivatives of the torsion and curvature tensor of $\nabla$.

4. Using (37) recursively then shows there exists $Q_{\ell, n}^{\nabla} \in \Gamma\left[\operatorname{Hom}\left[T M^{\otimes n}, T M^{\otimes \ell}\right]\right]$ such that

$$
\left\langle\nabla^{n} f, v_{n} \otimes \cdots \otimes v_{1}\right\rangle=\left\langle\operatorname{Sym} \nabla^{n} f, v_{n} \otimes \cdots \otimes v_{1}\right\rangle
$$




$$
+\sum_{\ell=1}^{n-1}\left\langle\operatorname{Sym} \nabla^{\ell} f, Q_{\ell, n}^{\nabla} v_{n} \otimes \cdots \otimes v_{1}\right\rangle,
$$

where each $Q_{\ell, n}^{\nabla}$ is constructed from certain combinations of covariant derivatives of the torsion and curvature tensor of $\nabla$.

\subsection{The polynomial regularity structure and model}

We are now ready to set up to regularity structure for "polynomials" up to order $n$ on a manifold.

Definition 77 Fix $n \geq 0$ and let $\mathcal{T}=\bigoplus_{\ell=0}^{n} \Sigma^{\ell} T^{*} M$ be the vector bundle over $M$ with fiber at $p \in M$ given by

$$
\left.\mathcal{T}\right|_{p}:=\bigoplus_{\ell=0}^{n} \Sigma^{\ell} T_{p}^{*} M .
$$

On each fiber of $\Sigma^{\ell} T^{*} M$ we use the norm induced by the Riemannian metric. With this norm, for $\tau \in \Sigma^{\ell} T_{p}^{*} M, X \in\left(T_{p} M\right)^{\otimes \ell}$

$$
|\langle\tau, X\rangle| \leq|\tau||X|
$$

The vector bundle $\mathcal{T}$ will be used to store higher order derivatives of functions. On flat space $\mathbb{R}^{d}$ such "abstract Taylor expansions" were realized as honest functions using polynomials, see (24). Polynomials are the simplest function that have specified derivatives at one point. On the manifold we instead choose polynomials in exponential coordinates.

Definition 78 (Realization of an abstract polynomial) For $\tau=\left(\tau_{0}, \ldots, \tau_{n}\right) \in \mathcal{T}_{p}$ define

$$
\begin{aligned}
\left(\Pi_{p} \tau\right)(z) & :=\left\langle\tau, \sum_{\ell=0}^{n}\left[\exp _{p}^{-1}(z)\right]^{\otimes \ell}\right\rangle \\
& =\sum_{\ell=0}^{n} \tau_{\ell}\left(\left[\exp _{p}^{-1}(z)\right]^{\otimes \ell}\right) .
\end{aligned}
$$

These local "Taylor polynomials" are a good substitute for the usual Taylor polynomials in the flat space theory, as Lemma 79 and Corollary 80 below demonstrate.

Lemma 79 Let $A=A_{0}+A_{1}+\cdots+A_{n} \in \mathcal{T}_{p}$, with $A_{\ell} \in \Sigma^{\ell} T_{p}^{*} M$, for $\ell=0, \ldots, n$, and define

$$
\varphi(q):=\Pi_{p} A=\sum_{\ell=0}^{n} A_{\ell}\left(\exp _{p}^{-1}(q)^{\otimes \ell}\right)
$$


Then,

$$
\varphi(p)=A_{0} \text { and } \operatorname{Sym}\left[\left.(\nabla)^{\ell} \varphi\right|_{p}\right]=\ell ! A_{\ell}, \forall \ell=1, \ldots, n \text {. }
$$

Proof Let $\gamma_{v}(t)=\exp _{p}(t v)$. Then,

$$
\varphi\left(\gamma_{v}(t)\right)=\sum_{\ell=0}^{n} A_{\ell}\left(\exp _{p}^{-1}\left(\gamma_{v}(t)^{\otimes \ell}\right)=\sum_{\ell=0}^{n} t^{\ell} A_{\ell}\left(v^{\otimes \ell}\right)\right.
$$

and hence by Lemma 57

$$
\nabla_{v^{\otimes \ell}}^{\ell} \varphi=\left.\frac{d^{\ell}}{d t^{\ell}}\right|_{t=0} \varphi\left(\gamma_{v}(t)\right)=\ell ! A_{\ell}\left(v^{\otimes \ell}\right)
$$

which suffices to complete the Proof by Remark 59.

We now have the immediate corollary of this lemma.

Corollary 80 Let $\tau \in \Sigma^{\ell} T_{p}^{*} M$. Then, for $i=0, \ldots, n$,

$$
\operatorname{Sym}\left[\nabla^{i}{ }_{p} \Pi_{p} \tau\right]= \begin{cases}\ell ! \tau, & i=\ell \\ 0, & \text { else. }\end{cases}
$$

Remark 81 Let $x$ be exponential coordinates around $p \in M$, i.e. suppose that $x=$ $\left(x^{1}, \ldots, x^{d}\right)$ where $\left\{x^{i}(q)\right\}_{i=1}^{d}$ are the coordinates of $\exp _{p}^{-1}(q)$ relative to some basis $\left\{u_{i}\right\}_{i=1}^{d}$ of $T_{p} M$. Then with $v=\sum_{i=1}^{d} v^{i} u_{i} \in T_{p} M$,

$$
\begin{aligned}
\sum_{i_{1} \ldots i_{\ell}=1}^{d} \partial_{i_{1} \ldots i_{\ell}} f(p) v^{i_{1}} \ldots v^{i_{\ell}} & =\left.\frac{d^{\ell}}{d t^{\ell}}\right|_{0} f\left(\exp _{p}(t v)\right)=\left\langle\left.\nabla\right|_{p} ^{\ell} f, v^{\otimes \ell}\right\rangle \\
& =\left\langle\operatorname{Sym}\left[\left.\nabla^{\ell}\right|_{p} f\right], v^{\otimes \ell}\right\rangle \\
& =\sum_{i_{1} \ldots i_{\ell}=1}^{d}\left\langle\operatorname{Sym}\left[\left.\nabla^{\ell}\right|_{p} f\right],\left(\frac{\partial}{\partial x^{i_{1}}}, \ldots, \frac{\partial}{\partial x^{i_{\ell}}}\right)\right\rangle v^{i_{1}} \ldots v^{i_{\ell}}
\end{aligned}
$$

from which it follows that

$$
\partial_{i_{1} \ldots i_{\ell}} f(p)=\operatorname{Sym}\left[\left.\nabla^{\ell}\right|_{p} f\right]\left(\frac{\partial}{\partial x^{i_{1}}}, \ldots, \frac{\partial}{\partial x^{i_{\ell}}}\right), \forall i_{1}, \ldots, i_{\ell}=1 \ldots d .
$$

Definition 82 (Transportation) Let $\Gamma_{p \leftarrow q}:\left.\left.\mathcal{T}\right|_{q} \rightarrow \mathcal{T}\right|_{p}$ be defined by $\Gamma_{p \leftarrow q} \tau:=\bar{\tau}$, where

$$
\bar{\tau}_{\ell}:=\frac{1}{\ell !} \operatorname{Sym}\left[\left.\nabla^{\ell}\right|_{p}\left(\Pi_{q} \tau\right)\right], \forall \ell=0, \ldots, n,
$$

which makes sense for $d(p, q)<\delta$, the radius of injectivity of $M$. 
Remark 83 For $n \geq 2$ this transport will in general also go "upwards." That is, if $\left.\tau \in \mathcal{T}_{\alpha}\right|_{y}$ some $\alpha<n$, then in general $\Gamma_{x \leftarrow y} \tau$ will have components in homogeneities strictly larger than $\alpha$. This is not allowed in the original formulation of a regularity structure by Hairer [9, Definition 2.1]. As we have seen in the main text, this poses no problem, since our modified definition of a model (Definition 14) allows for it. We moreover believe that any transport that wants to achieve the following lemma for a "polynomial model" is forced to do this.

The definitions have been arranged so that $\Pi_{q} \tau$ and $\Pi_{p} \Gamma_{p \leftarrow q} \tau$ agree at $p$ to order $n$ :

Lemma 84 Let $\left.\tau \in \mathcal{T}\right|_{q}$ and $p, z \in U$ where $U$ is a sufficiently small neighborhood of $q$. If $V$ is a differential operator of order $k \leq n$ defined on $U$, then

$$
\left|V\left[\Pi_{q} \tau-\Pi_{p} \Gamma_{p \leftarrow q} \tau\right](z)\right| \lesssim_{V}|\tau| d(z, p)^{n+1-k} .
$$

Proof Let

$$
g(z)=\left(\Pi_{q} \tau\right)(z)
$$

so that

$$
\left(\Pi_{p} \Gamma_{p \leftarrow q} \tau\right)(z)=\left(\operatorname{Tay}_{p}^{n} g\right)(z)
$$

where $\operatorname{Tay}_{p}^{n}$ was defined in Definition 65. Using Corollary 69, we have the estimate,

$$
\begin{aligned}
& \left\|\left.\nabla^{k}\right|_{z}\left[g-\left(\operatorname{Tay}_{p}^{n} g\right)\right]\right\| \\
& \quad \leq \frac{1}{(n+1-k) !} \max _{0 \leq t \leq 1}\left\|\left.\nabla^{n+1}\right|_{\gamma_{v}(t)}\left(g-\operatorname{Tay}_{p}^{n} g\right)\right\| \cdot d(p, z)^{n+1-k},
\end{aligned}
$$

where $v:=\exp _{p}^{-1}(z)$. For $d(z, p)<\varepsilon$ and $0 \leq t \leq 1$, let $[p, z]_{t}:=$ $\exp \left(t \exp _{p}^{-1}(z)\right)$ so that $t \rightarrow[p, z]_{t}$ is the geodesic joining $p$ to $z$ parametrized by $[0,1]$. Then we have

$$
\begin{aligned}
\max _{0 \leq t \leq 1}\left\|\left.\nabla^{n+1}\right|_{\gamma_{v}(t)}\left(g-\operatorname{Tay}_{p}^{n} g\right)\right\| & =\max _{0 \leq t \leq 1}\left\|\nabla^{n+1} \mid[p, z]_{t}\left(g-\operatorname{Tay}_{p}^{n} g\right)\right\| \\
& \leq \max _{w: d(w, p) \leq d(p, z)}\left\|\left.\nabla^{n+1}\right|_{w}\left(g-\operatorname{Tay}_{p}^{n} g\right)\right\|
\end{aligned}
$$

and so we have

$$
\begin{aligned}
& \left\|\left.\nabla^{k}\right|_{z}\left[g-\left(\operatorname{Tay}_{p}^{n} g\right)\right]\right\| \\
& \leq \frac{1}{(n+1-k) !} \max _{w: d(w, p) \leq d(p, z)} \|\left.\nabla^{n+1}\right|_{w}\left(g-\text { Tay }_{p}^{n} g\right) \| \cdot d(p, z)^{n+1-k} .
\end{aligned}
$$


For the proof of the first half of Theorem 92 below, it is convenient to introduce as in [5] the notion of a parallelism on a vector bundle, $E$, over $M$.

Definition 85 (Diagonal domains) Let $\mathcal{U}$ be an open set on $M$. An open set $\mathrm{D}^{\mathcal{U}} \subseteq$ $M \times M$ is a $\mathcal{U}$ - diagonal domain if it contains the diagonal of $\mathcal{U}$, that is $\Delta^{\mathcal{U}}:=$ $\bigcup_{p \in \mathcal{U}}(p, p) \subseteq \mathrm{D}^{\mathcal{U}}$. A local diagonal domain is a $\mathcal{V}$ - diagonal domain for some nonempty open $\mathcal{V} \subseteq M$.

If $\mathcal{U}=M$, we write $\mathrm{D}:=\mathrm{D}^{M}$ and refer to $\mathrm{D}$ simply as a diagonal domain.

Definition 86 (Parallelisms) Let $E$ be a vector bundle over $M$ and Hom $(E) \rightarrow M \times M$ be the associated vector bundle over $M \times M$ with fibers, $\operatorname{Hom}_{(q, p)}(E):=L\left(E_{p}, E_{q}\right)$ for $(q, p) \in M \times M$, where $L\left(E_{p}, E_{q}\right)$ denote the set of all linear transformations from $E_{p}$ to $E_{q}$. A smooth local section $U \in \Gamma(\operatorname{Hom}(E))$ with domain D (i.e. $U(q, p) \in$ $L\left(E_{p}, E_{q}\right)$ for all $\left.(q, p) \in \mathrm{D}\right)$ is called a parallelism if $U(p, p)=\mathrm{id}_{p}$. If $U$ is only defined on a local diagonal domain, we refer to $U$ as a local parallelism.

Example 87 (Parallel translation and parallelisms) One natural example of a parallelism when $(M, g)$ is a Riemannian manifold and $E$ is equipped with a covariant derivative, $\nabla^{E}$, is to define

$$
U^{\nabla}(q, p):=/ /_{1}^{E}\left(t \rightarrow \exp _{p}\left(t \exp _{p}^{-1}(q)\right)\right)
$$

where $p, q \in M$ are "close enough" so there is a unique vector $v_{p}$ with minimum length such that $q=\exp _{p}\left(v_{p}\right)$ and $/\left.\right|_{(\cdot)} ^{E}$ denotes the parallel translation operator on $E$ relative to $\nabla^{E}$. For our purposes below $E$ will be a bundle associated to $T M$ and $\nabla^{E}$ will be the induced connection on this bundle associated to the Levi-Civita covariant derivative on $(M, g)$.

Example 88 (Charts and parallelisms) Each chart $(\Psi, \mathcal{U})$ induces a local parallelism on $\left(T^{*} M\right)^{\otimes \ell}$ for any $\ell \in \mathbb{N}$ as follows. If $A \in\left(T_{p}^{*} M\right)^{\otimes \ell}$ is expressed as

$$
A=\left.\left.\sum_{i_{1}, \ldots i_{\ell}=1}^{d} A_{i_{1}, \ldots, i_{\ell}} d \Psi^{i_{1}}\right|_{p} \otimes \cdots \otimes d \Psi^{i_{\ell}}\right|_{p}
$$

then we define $U^{\Psi}(q, p) A \in T_{q}^{*} M^{\otimes \ell}$ by

$$
U^{\Psi}(q, p) A:=\left.\left.\sum_{i_{1}, \ldots i_{\ell}=1}^{d} A_{i_{1}, \ldots, i_{\ell}} d \Psi^{i_{1}}\right|_{q} \otimes \cdots \otimes d \Psi^{i_{\ell}}\right|_{q}
$$

In other words, $U^{\Psi}(q, p)$ is uniquely determined by requiring

$$
\left\langle U^{\Psi}(q, p) A,\left.\left.\frac{\partial}{\partial \Psi^{i_{1}}}\right|_{q} \otimes \cdots \otimes \frac{\partial}{\partial \Psi^{i_{\ell}}}\right|_{q}\right\rangle=\left\langle A,\left.\left.\frac{\partial}{\partial \Psi^{i_{1}}}\right|_{p} \otimes \cdots \otimes \frac{\partial}{\partial \Psi^{i_{\ell}}}\right|_{p}\right\rangle
$$

for all $q \in \mathcal{U}$ and $1 \leq i_{1}, i_{2}, \ldots i_{\ell} \leq d$. [This example is basically a special case of Example 87 where one takes $\nabla$ to be the flat connection, $D^{\Psi}$, defined in Definition 70.$]$ 
With the aid of a parallelism, we can now define the notion of $\gamma-$ Hölder section, $S$, on $E$. In what follows we assume that $E$ is equipped with a smoothly varying inner product, $\langle\cdot, \cdot\rangle_{E}$. We do not necessarily assume that $\nabla^{E}$ is compatible with $\langle\cdot, \cdot\rangle_{E}$ or that $U(p, q)$ is unitary for all $(p, q) \in \mathrm{D}$.

Lemma 89 Let $S$ be a continuous section of a vector bundle E. Let $(U, D),\left(U^{\prime}, D\right)$ be parallelisms on $E$. Then for every compactum $K \subset D$

$$
\|U(q, p) S(p)-S(q)\| \leq C_{K}\left(\left\|U^{\prime}(q, p) S(p)-S(q)\right\|+d(p, q)\right), \forall p, q \in K .
$$

Proof We work in a local trivialization. Let $U, U^{\prime}: \mathbb{R}^{d} \times \mathbb{R}^{d} \rightarrow G L\left(\mathbb{R}^{N}\right)$ be smooth functions such that $U(x, x), U^{\prime}(x, x)=\mathrm{id}$, which we view to be a parallelism on the trivial bundle, $\mathbb{R}^{d} \times \mathbb{R}^{N}$ over $\mathbb{R}^{d}$. A continuous section of this bundle may be identified with a continuous function, $S: \mathbb{R}^{d} \rightarrow \mathbb{R}^{N}$ Then

$\|U(x, y) S(y)-S(x)\| \leq\left\|\left(U(x, y)-U^{\prime}(x, y)\right) S(y)\right\|+\left\|U^{\prime}(x, y) S(y)-S(x)\right\|$.

The statement then follows from smoothness of $U, U^{\prime}$, the fact that they coincide at $x, x$ and local boundedness of $S$.

Lemma 90 Let $f \in C(M), \gamma>0$ and $n=\lfloor\gamma\rfloor \in \mathbb{N}_{0}$. Then $f \in C^{\gamma}(M)$ (as in Definition 10) iff is $f$ a $n$-times continuously differentiable function on $M$ and for any (local) parallelism $U$ on the vector bundle $\Sigma^{n} T^{*} M, \operatorname{Sym}\left[\nabla^{n} \mid f\right]$ satisfies

$$
\left|U(q, p) \operatorname{Sym}\left[\left.\nabla^{n}\right|_{p} f\right]-\operatorname{Sym}\left[\left.\nabla^{n}\right|_{q} f\right]\right| \lesssim d(q, p)^{\gamma-n} .
$$

Proof Recall from Definition 10, that $f \in C(M)$ is in $C^{\gamma}(M)$ iff $f \circ \Psi^{-1} \in C^{\gamma}(\Psi(\mathcal{U}))$ for every coordinate chart $(\Psi, \mathcal{U})$. These conditions are equivalent to $f$ being $n$-times continuously differentiable and the $n^{\text {th }}$ - derivatives of $f \circ \Psi^{-1}$ being locally $(\gamma-n)$ Hölder on $\Psi(\mathcal{U})$. The latter condition may be expressed as saying

$$
\left|U^{\Psi}(q, p) D^{n}\right|_{p} f-\left.D^{n}\right|_{q} f \mid \lesssim d(q, p)^{\gamma-n}
$$

where $D=D^{\Psi}$ is the flat connection defined in Notation 70. From Lemma 72 and Corollary 73 we may express

$$
D^{n} f=\operatorname{Sym}\left[\nabla^{n} f\right]+L f
$$

where $L$ is a linear differential operator of order at most $n-1$. As $L f$ is continuously differentiable it follows that

$$
(q, p) \rightarrow U^{\Psi}(q, p)(L f)_{p}-(L f)_{q}
$$

is continuously differentiable and vanishes at $q=p$ and therefore (by the fundamental theorem of calculus)

$$
\left|U^{\Psi}(q, p)(L f)_{p}-(L f)_{q}\right| \lesssim d(q, p)
$$


From (41) and (42) it follows that (40) is equivalent to

$$
\left|U^{\Psi}(q, p) \operatorname{Sym}\left[\left.\nabla^{n}\right|_{p} f\right]-\operatorname{Sym}\left[\left.\nabla^{n}\right|_{q} f\right]\right| \lesssim d(q, p)^{\gamma-n} .
$$

Lastly using Lemma 89 we conclude that the estimates in (43) and (39) are also equivalent.

Theorem 91 Fix $n \in \mathbb{N}_{0}$ and construct $\mathcal{T}$ and $(\Pi, \Gamma)$ as above. Then $\mathcal{T}$ is a regularity structure (in the sense of Definition 13$)$ and $(\Pi, \Gamma)$ is a model of transport precision $n+1$ (in the sense of Definition 14).

Proof The fact that $\mathcal{T}$ is a regularity structure is immediate. Let us now set $\delta_{M}=\delta$ to be the injectivity radius of $M$ and for $q \in M$, let $U_{q}:=\exp _{q}\left(B_{T_{q} M}\left(0_{q}, \delta_{M}\right)\right)$.

We have to check that

$$
\|(\Pi, \Gamma)\|_{\beta ; M}<\infty .
$$

The homogeneity estimate, $\left|\left\langle\Pi_{p} \tau, \varphi_{p}^{\lambda}\right\rangle\right| \lesssim \lambda^{\ell}$, for $\left.\tau \in \mathcal{T}_{\ell}\right|_{p}$ follows from the fact that $\Pi_{p} \tau$ is a monomial of order $\ell$ in $\exp _{p}^{-1}$-coordinates. Lemma 84 gives the transport precision, i.e.

$$
\left|\left\langle\Pi_{q} \tau-\Pi_{p} \Gamma_{p \leftarrow q} \tau, \varphi_{p}^{\lambda}\right\rangle\right| \lesssim \lambda^{n+1} \text { for all }\left.\tau \in \mathcal{T}\right|_{q}
$$

Let $D$ be the covariant derivative induced by the chart $\exp _{q}^{-1}$. Using Lemma 72 we get

$$
\begin{aligned}
\left|\left\langle\operatorname{Sym}\left[\left.\nabla^{m}\right|_{p} \tau_{\ell}\left(\left(\exp _{q}^{-1}\right)^{\otimes \ell}\right)\right], W\right\rangle\right| & =\left|\left\langle\left.\nabla^{m}\right|_{p} \tau_{\ell}\left(\left(\exp _{q}^{-1}\right)^{\otimes \ell}\right), \operatorname{Sym}[W]\right\rangle\right| \\
& =\left|\sum_{i=1}^{m}\left\langle\left. D^{i}\right|_{p} \tau_{\ell}\left(\left(\exp _{q}^{-1}\right)^{\otimes \ell}\right), Q_{i, m} \operatorname{Sym}[W]\right\rangle\right| \\
& \lesssim \sum_{i=1}^{m} d(p, q)^{\ell-i}|| \tau|||W| \\
& \lesssim d(p, q)^{\ell-m}|| \tau|||W|,
\end{aligned}
$$

and hence $\left\|\Gamma_{p \leftarrow q} \tau_{\ell}\right\|_{m} \lesssim d(p, q)^{\ell-m}$, which finishes the proof.

We are finally able to characterize $C^{\gamma}(M)$ in terms of the "polynomial" regularity structure.

Theorem 92 Let $\gamma \in(0, \infty) \backslash \mathbb{N}$ and $f: M \rightarrow \mathbb{R}$ a continuous function. Then, $f \in C^{\gamma}(M)$ if and only if there is $\hat{f} \in \mathscr{D}^{\gamma}(M, \mathcal{T})^{13}$ with $\hat{f}_{0}(p)=f(p)$. In that case,

$$
\hat{f}_{\ell}(p)=\frac{1}{\ell !} \operatorname{Sym}\left[\left.\nabla^{\ell}\right|_{p} f\right]
$$

\footnotetext{
13 The space of modelled distributions was defined in Definition 18.
} 
Proof $(\Longrightarrow)$ Let $f \in C^{\gamma}(M)$ and define

$$
\hat{f}(p):=\sum_{\ell=0}^{\lfloor\gamma\rfloor} \frac{1}{\ell !} \operatorname{Sym}\left[\left.\nabla^{\ell}\right|_{p} f\right],
$$

i.e. $\hat{f}_{\ell}(p):=\operatorname{Sym}\left[\left.\nabla^{\ell}\right|_{p} f\right]$ for $0 \leq \ell \leq\lfloor\gamma\rfloor=$ : $n$. We have to check that $\hat{f} \in$ $\mathscr{D}^{\gamma}(M, \mathcal{T})$, i.e. for all $\ell \leq\lfloor\gamma\rfloor$ and $d(p, q)<\delta$

$$
\left\|\hat{f}(q)-\Gamma_{q \leftarrow p} \hat{f}(p)\right\|_{\ell} \lesssim d(p, q)^{\gamma-\ell}
$$

or equivalently, using the definition of $\Gamma_{q \leftarrow p}$, if

$$
g(q):=\left(\Pi_{p} \hat{f}(p)\right)(q),
$$

we must show

$$
\left|\operatorname{Sym}\left[\left.\nabla^{\ell}\right|_{q}(f-g)\right]\right| \lesssim d(p, q)^{\gamma-\ell} .
$$

$\ell=n:$

Recall $\gamma-n \in(0,1]$. Now the term to bound in (44) reads as

$$
\begin{gathered}
\mid \operatorname{Sym}\left[\left.\nabla^{n}\right|_{q} f-\left.\nabla^{n}\right|_{q}\left[\sum_{i=0}^{n}\left\langle\left.\nabla^{i}\right|_{p} f, \exp _{p}^{-1}(\cdot)^{\otimes i}\right]\right] \mid\right. \\
\leq \mid \operatorname{Sym}\left[\left.\nabla^{n}\right|_{q} f-\left.\nabla^{n}\right|_{q}\left[\left\langle\left.\nabla^{n}\right|_{p} f, \exp _{p}^{-1}(\cdot)^{\otimes n}\right]\right] \mid\right. \\
+\sum_{i=0}^{n-1} \mid \operatorname{Sym}\left[\left.\nabla^{n}\right|_{q}\left[\left\langle\left.\nabla^{i}\right|_{p} f, \exp _{p}^{-1}(\cdot)^{\otimes i}\right]\right] \mid .\right.
\end{gathered}
$$

By Lemma 80

$$
\operatorname{Sym}\left[\left.\nabla^{n}\right|_{q}\left[\left\langle\left.\nabla^{i}\right|_{p} f, \exp _{p}^{-1}(\cdot)^{\otimes i}\right]\right]=0 \text {, at } q=p,\right.
$$

and since the expression is smooth in $q$ we can focus on

$$
\mid \operatorname{Sym}\left[\left.\nabla^{n}\right|_{q} f-\left.\nabla^{n}\right|_{q}\left[\left\langle\left.\nabla^{n}\right|_{p} f, \exp _{p}^{-1}(\cdot)^{\otimes n}\right]\right] \mid\right.
$$

Define on the vector bundle $\Sigma^{n} T^{*} M$ the parallelism

$$
U(q, p) S:=\left.\nabla^{n}\right|_{q}\left\langle S, \exp _{p}^{-1}(\cdot)^{\otimes n}\right\rangle
$$

Then by Lemma 90

$$
\left|\operatorname{Sym}\left[\left.\nabla^{n}\right|_{q} f\right]-U(q, p) \operatorname{Sym}\left[\left.\nabla^{n}\right|_{p} f\right]\right| \lesssim d(q, p)^{\gamma-n},
$$


so for $\ell=n$ we are done.

$\ell=0, \ldots, n-1$ : We need to show (44). It is enough to bound for $w \in T_{p} M$, with $v:=\exp _{p}^{-1}(q)$

$$
\left|\left\langle\left.\nabla^{\ell}\right|_{q}(f-g),\left(/ /_{t}\left(\gamma_{v}\right) w\right)^{\otimes \ell}\right\rangle\right| \lesssim|w|^{\ell} d(p, q)^{\gamma-\ell}
$$

Here $/ /_{t}\left(\gamma_{v}\right): T_{\gamma_{v}(0)} M \rightarrow T_{\gamma_{v}(t)} M$ denotes the parallel transport along $\gamma_{v}(t):=$ $\exp _{p}(t v)$.

For this purpose, define

$$
W_{t}:=/ /_{t}\left(\gamma_{v}\right) w_{1} \otimes \cdots \otimes / / t\left(\gamma_{v}\right) w_{\ell}
$$

and $F:=f-g$. Since $W_{t}$ and $\dot{\gamma}_{v}(t)$ are parallel along $\gamma_{v}(t)$ it follows that

$$
\frac{d^{k}}{d t^{k}} \nabla_{W_{t}}^{\ell} F=\nabla_{\dot{\gamma}_{v}(t)^{\otimes k} \otimes W_{t}}^{\ell+k} F \forall 0 \leq k \leq n-\ell .
$$

Therefore by Taylor's theorem and the fact that $\left.\nabla^{m}\right|_{p} F_{p}=0$ for $0 \leq m \leq n,{ }^{14}$ we have

$$
\begin{aligned}
\nabla_{W_{1}}^{\ell} F & =\sum_{k=0}^{n-\ell-1} \frac{1}{k !} \nabla_{v^{\otimes k} \otimes W_{0}}^{\ell+k} F+\frac{1}{(n-\ell-1) !} \int_{0}^{1}\left[\nabla_{\dot{\gamma}_{v}(t)^{\otimes n-\ell} \otimes W_{t}}^{n} F\right] \cdot(1-t)^{n-\ell-1} d t \\
& =\frac{1}{(n-\ell-1) !} \int_{0}^{1}\left[\nabla_{\dot{\gamma}_{v}(t)^{\otimes n-\ell} \otimes W_{t}}^{n} F\right] \cdot(1-t)^{n-\ell-1} d t
\end{aligned}
$$

Since $g$ is smooth we apply the fundamental theorem of calculus to find

$$
\begin{aligned}
\nabla_{\dot{\gamma}_{v}(t)^{\otimes(n-\ell)} \otimes W_{t}}^{n} g & =\nabla_{v^{\otimes(n-\ell)} \otimes W_{0}}^{n} g+\int_{0}^{t} \nabla_{\dot{\gamma}_{v}(\tau)^{\otimes(n-\ell)+1} \otimes W_{\tau}}^{n+1} g d \tau \\
& =\nabla_{v^{\otimes(n-\ell)} \otimes W_{0}}^{n} f+\int_{0}^{t} \nabla_{\dot{\gamma}_{v}(\tau)^{\otimes(n-\ell)+1} \otimes W_{\tau}}^{n+1} g d \tau \\
& =\nabla_{v^{\otimes(n-\ell)} \otimes W_{0}}^{n} f+O\left(|v|^{(n-\ell)+1}\left|W_{0}\right|\right) .
\end{aligned}
$$

Using this estimate, it follows that

$$
\nabla_{\dot{\gamma}_{v}(t)^{\otimes(n-\ell)} \otimes W_{t}}^{n} F=\nabla_{\dot{\gamma}_{v}(t)^{\otimes(n-\ell)} \otimes W_{t}}^{n} f-\nabla_{v^{\otimes(n-\ell)} \otimes W_{0}}^{n} f+O\left(|v|^{(n-\ell)+1}\left|W_{0}\right|\right) .
$$

Since

$$
\begin{aligned}
\nabla_{\dot{\gamma}_{v}(t)^{\otimes(n-\ell)} \otimes W_{t}}^{n} f & =\left.\nabla^{n}\right|_{\gamma_{v}(t)} f\left(/ /_{t}\left(\gamma_{v}\right) v\right)^{\otimes(n-\ell)} \otimes\left(/ /_{t}\left(\gamma_{v}\right) w\right)^{\otimes \ell} \\
& =\left\langle\left. U\left(p, \gamma_{v}(t)\right) \nabla^{n}\right|_{\gamma_{v}(t)} f, v^{\otimes(n-\ell)} w^{\otimes \ell}\right\rangle .
\end{aligned}
$$

$\overline{14}$ This follows by the very construction of $g$ along with Corollary 74 and Lemma 80. 
As shown in the step $\ell=n$, we then get $\left|\nabla_{\dot{\gamma}_{v}(t)^{\otimes(n-\ell)} \otimes W_{t}}^{n} f-\nabla_{v^{\otimes(n-\ell)} \otimes W_{0}}^{n} f\right| \leq C d\left(\gamma_{v}(t), p\right)^{\gamma-n}|v|^{n-\ell}|w|^{\ell}=C|v|^{\gamma-\ell}|w|^{\ell}$. and hence

$$
\left|\nabla_{\dot{\gamma}_{v}(t)^{\otimes(n-\ell)} \otimes W_{t}}^{n} F\right| \leq\left[C|v|^{\gamma-\ell}+O\left(|v|^{n-\ell+1}\right)\right]|w|^{\ell} \leq C^{\prime}|v|^{\gamma-\ell}|w|^{\ell} .
$$

Plugging this estimate back into (46) shows,

$$
\left|\nabla_{W_{1}}^{\ell} F\right| \leq C|v|^{\gamma-\ell}|w|^{\ell}
$$

which completes the proof of (44).

$(\Longleftarrow)$

Recall that $\gamma \in(n, n+1]$, for some $n \in \mathbb{N}_{0}$.

Step 1: We will show that $f$ is $n$-times differentiable and $\frac{1}{\ell !} \operatorname{Sym}\left[\nabla^{\ell} f\right]=\hat{f}_{\ell}$ for $\ell=0, \ldots, n$. This will be done by induction.

So assume for some $\ell=0, \ldots, n-1$ we know that

- $f$ is $\ell$-times differentiable

- $\frac{1}{i !} \operatorname{Sym}\left[\nabla^{i} f\right]=\hat{f}_{i}, \quad i=0, \ldots, \ell$

By Taylor's theorem (Theorem 62)

$$
\begin{aligned}
f\left(\exp _{p}(v)\right)= & \sum_{j=0}^{\ell-1} \frac{1}{j !} \operatorname{Sym}\left[\left.\nabla^{j}\right|_{p} f\right]\left(v^{\otimes j}\right) \\
& +\frac{1}{(\ell-1) !} \int_{0}^{1}(1-t)^{\ell-1} \operatorname{Sym}\left[\left.\nabla^{\ell}\right|_{\gamma_{v}(t)} f\right]\left(\dot{\gamma}_{v}(t)^{\otimes \ell}\right) .
\end{aligned}
$$

Now by assumption

$$
\left|\operatorname{Sym}\left[\left.\nabla^{\ell}\right|_{q}(f-g)\right]\right| \lesssim d(q, p)^{\gamma-\ell},
$$

where

$$
g(q):=\left(\Pi_{p} \hat{f}(p)\right)(q)
$$

Hence

$$
\operatorname{Sym}\left[\left.\nabla^{\ell}\right|_{\gamma_{v}(t)} f\right]=\operatorname{Sym}\left[\left.\nabla^{\ell}\right|_{\gamma_{v}(t)} g\right]+O\left(|t v|^{\gamma-\ell}\right) .
$$

Plugging this into (47) and using the fact that $\left|\dot{\gamma}_{v}(t)\right|=|v|$ we get

$$
f\left(\exp _{p}(v)\right)=\sum_{j=0}^{\ell-1} \frac{1}{j !} \operatorname{Sym}\left[\left.\nabla^{i}\right|_{p} f\right]\left(v^{\otimes j}\right)
$$




$$
+\frac{1}{(\ell-1) !} \int_{0}^{1}(1-t)^{\ell-1} \operatorname{Sym}\left[\left.\nabla^{\ell}\right|_{\gamma_{v}(t)} g\right]\left(\dot{\gamma}_{v}(t)^{\otimes \ell}\right)+O\left(|v|^{\gamma}\right) .
$$

Now, since $g$ is smooth and $\frac{\nabla}{d t} \dot{\gamma}_{v}(t)=0$, we have

$$
\begin{aligned}
& \frac{d}{d t}\left[\left.\nabla^{\ell}\right|_{\gamma_{v}(t)} g\left(\dot{\gamma}_{v}(t)^{\otimes \ell}\right)\right]=\left.\nabla^{\ell+1}\right|_{\gamma_{v}(t)} g\left(\dot{\gamma}_{v}(t)^{\otimes(\ell+1)}\right) \text { and } \\
& \frac{d^{2}}{d t^{2}}\left[\left.\nabla^{\ell}\right|_{\gamma_{v}(t)} g\left(\dot{\gamma}_{v}(t)^{\otimes \ell}\right)\right]=\left.\nabla^{\ell+2}\right|_{\gamma_{v}(t)} g\left(\dot{\gamma}_{v}(t)^{\otimes(\ell+2)}\right)
\end{aligned}
$$

and therefore by Taylor's theorem (in one variable) together with Lemma 57

$$
\begin{aligned}
& \operatorname{Sym}\left[\left.\nabla^{\ell}\right|_{\gamma_{v}(t)} g\right]\left(\dot{\gamma}_{v}(t)^{\otimes \ell}\right) \\
& =\left[\left.\nabla^{\ell}\right|_{\gamma_{v}(t)} g\right]\left(\dot{\gamma}_{v}(t)^{\otimes \ell}\right) \\
& =\left[\left.\nabla^{\ell}\right|_{p} g\right]\left(v^{\otimes \ell}\right)+t\left[\left.\nabla^{\ell+1}\right|_{p} g\right]\left(v^{\otimes(\ell+1)}\right)+O\left(|t v|^{\ell+2}\right) \\
& =\operatorname{Sym}\left[\left.\nabla^{\ell}\right|_{p} f\right]\left(v^{\otimes \ell}\right)+t \operatorname{Sym}\left[\left.\nabla^{\ell+1}\right|_{p} g\right]\left(v^{\otimes(\ell+1)}\right)+O\left(|t v|^{\ell+2}\right) \\
& =\operatorname{Sym}\left[\left.\nabla^{\ell}\right|_{p} f\right]\left(v^{\otimes \ell}\right)+t(\ell+1) ! \hat{f}_{\ell+1}(p)\left(v^{\otimes(\ell+1)}\right)+O\left(|t v|^{\ell+2}\right)
\end{aligned}
$$

A simple integration by parts argument shows

$$
\frac{1}{k !} \int_{0}^{1}(1-t)^{k} t d t=\frac{1}{(k+2) !}
$$

Combining (48), (49) and (50), we get

$$
\begin{aligned}
f\left(\exp _{p}(v)\right)= & \sum_{j=0}^{\ell-1} \frac{1}{j !} \operatorname{Sym}\left[\left.\nabla^{i}\right|_{p} f\right]\left(v^{\otimes j}\right)+\frac{1}{\ell !} \operatorname{Sym}\left[\left.\nabla^{\ell}\right|_{p} f\right]\left(v^{\otimes \ell}\right) \\
& +\hat{f}_{\ell+1}(p)\left(v^{\otimes(\ell+1)}\right)+O\left(|v|^{\gamma}\right) .
\end{aligned}
$$

As $v \mapsto \exp _{p}(v)$ is a local diffeomorphism, it now follows from (51) that $f$ is $\ell+1$ times differentiable at $p$ and moreover since,

$$
f\left(\exp _{p}(t v)\right)=\sum_{j=0}^{\ell} \frac{t^{j}}{j !} \operatorname{Sym}\left[\left.\nabla^{i}\right|_{p}\right] f\left(v^{\otimes j}\right)+t^{\ell+1} \hat{f}_{\ell+1}(p)\left(v^{\otimes(\ell+1)}\right)+O\left(|t|^{\gamma}\right)
$$

we may conclude, using Lemma 57 that

$$
\left.\nabla^{\ell+1}\right|_{p} f\left(v^{\otimes(\ell+1)}\right)=\left.\frac{d^{\ell+1}}{d t^{\ell+1}}\right|_{t=0} f\left(\exp _{p}(t v)\right)=(\ell+1) ! \hat{f}_{\ell+1}(p)\left(v^{\otimes(\ell+1)}\right) .
$$


Then by Remark 59 it follows that

$$
\frac{1}{(\ell+1) !} \operatorname{Sym}\left[\nabla^{\ell+1} f\right]_{p}=\hat{f}_{\ell+1}(p) .
$$

Step 2: So far we have shown that $f$ is $n$-times continuously differentiable and that $\operatorname{Sym}\left[\left.\nabla^{\ell}\right|_{p} f\right]=\hat{f}_{\ell}(p)$ for $\ell=0, \ldots, n$. Then with $U$ defined in (45) we have

$$
\left|\operatorname{Sym}\left[\left.\nabla^{n}\right|_{q} f\right]-U(q, p) \operatorname{Sym}\left[\left.\nabla^{n}\right|_{p} f\right]\right| \leq \| \hat{f}(q)-\Gamma_{q \leftarrow p} \hat{f}(p)||_{n}+\left.\left|\sum_{\ell \leq n-1}\right| \nabla^{n}\right|_{q} \Pi_{p} \hat{f}_{\ell}(p) \mid .
$$

The second to last term is of order $d(q, p)^{\gamma-n}$ by assumption. Moreover, for $\ell \leq$ $n-1$, by Corollary 80, we have $\left.\nabla^{n}\right|_{p} \Pi_{p} \hat{f}_{\ell}(p)=0$. Hence the last term is of order $d(q, p) \lesssim d(q, p)^{\gamma-n}$. By Lemma 90 we hence get that $f \in C^{\gamma}(M)$.

\subsection{A reformulation: the jet bundle}

In this section we briefly outline that the "polynomial" regularity structure is in fact (isomorphic to) the jet bundle. In anticipation of possible future work with vector bundle valued equations, we formulate this in the general setting of a vector bundle $E \stackrel{\pi}{\rightarrow} M$ with model fiber being a real finite dimensional vector space $V$. The connection to the previous sections is proven in Theorem 99 . We then conclude by showing Taylor's theorem in this setting, Theorem 103. We leave the complete construction of a polynomial regularity structure and its model in this fibered setting to future work. For background on vector bundles, we refer to [13, Chapter 10] and [16].

For each $m \in M$, let $\Gamma(m)$ be the germ of $C^{\infty}$-local sections of $E$ whose domain contains $m$. Fixing an integer $n \in \mathbb{N}_{0}$, we define an equivalence relation on $\Gamma(m)$ as follows. Let $(x, u)$ be a chart and local frame such that $m \in \operatorname{dom}(x)=\operatorname{dom}(u)$. We say $S, T \in \Gamma(m)$ are equivalent and write $S \stackrel{n}{\sim} T$ provided

$$
\left(\partial_{x}^{\alpha}\left[u^{-1}(S-T)\right]\right)(m)=0 \text { for all }|\alpha| \leq n,
$$

where $u^{-1}(p):=u(p)^{-1}: E_{p} \rightarrow V$ is the inverse of the linear operator, $u(p)$ : $V \rightarrow E_{p}$. It is well known and easy to check that " $\stackrel{n}{\sim}$ " is an equivalence relation which (by the chain and product rules) is independent of the choice of chart and local frame $(x, u)$ as above.

The equivalence relation in (52) may also be written as $S \stackrel{n}{\sim} T$ provided

$$
D^{k}\left[\left(u^{-1} S-u^{-1} T\right) \circ x^{-1}\right](x(m))=0 \text { for } 0 \leq k \leq n
$$

where for an open subset, $U \subset \mathbb{R}^{d}, a \in U$, and $g \in C^{\infty}(U, V),\left(D^{k} g\right)(a)$ is the $k$-linear form on $\mathbb{R}^{d}$ defined by

$$
\left(D^{k} g\right)(a)\left(v_{1}, \ldots, v_{k}\right)=\left(\partial_{v_{1}} \ldots \partial_{v_{k}} g\right)(a) \forall v_{1}, \ldots, v_{k} \in \mathbb{R}^{d} .
$$


As mixed partial derivatives commute, $\left(D^{k} g\right)(a)$ is symmetric and hence $\left(D^{k} g\right)(a)$ is completely determined by its values on the diagonal, $v_{1}=\cdots=v_{k}=v$ and thus we may also write $S \stackrel{n}{\sim} T$ provided

$$
\begin{gathered}
\left(\partial_{v}^{k}\left[\left(u^{-1} S-u^{-1} T\right) \circ x^{-1}\right]\right)(x(m)):=D^{k}\left[\left(u^{-1} S-u^{-1} T\right) \circ x^{-1}\right](x(m))\left(v^{\otimes k}\right) \\
=0 \text { for } 0 \leq k \leq n \text { and } v \in \mathbb{R}^{d} .
\end{gathered}
$$

Definition 93 Given $n \in \mathbb{N}_{0}, m \in M$, and $S \in \Gamma(m)$, let $j_{m}^{n} S$ be the $n$-jet of $S$ at $m$ defined to be the equivalence class

$$
j_{m}^{n} S:=\{T \in \Gamma(m): S \stackrel{n}{\sim} T\} .
$$

The $n$-jet bundle of $E$, denoted by $J^{n}(\pi)$, is the vector bundle whose fiber over $m \in M$ is $J_{m}^{n}(\pi):=\left\{j_{m}^{n} S: S \in \Gamma(m)\right\}$.

We will identity $J^{n}(\pi)$ with the vector bundle $\oplus_{k=0}^{n} \Sigma^{k}\left(T^{*} M\right) \otimes E$. The identification that we will give is not canonical but will depend on choosing covariant derivatives, $\nabla^{E}$ and $\nabla^{M}$ on $E$ and $T M$ respectively. We now fix such a pair of covariant derivatives. We denote by $\frac{\nabla^{E}}{d t}$ (resp. $\frac{\nabla^{M}}{d t}$ ) the covariant derivative of sections of $E$ (resp. $T M$ ) along curves in $M$.

Definition 94 For $v \in T_{m} M$, let $\sigma_{v}(t):=\exp (t v):=\exp ^{\nabla^{M}}(t v)$ so that

$$
\frac{\nabla^{M}}{d t} \dot{\sigma}_{v}(t)=0 \text { and } \dot{\sigma}_{v}(0)=v \in T_{m} M
$$

By the inverse function theorem, there exists an open ball $B \subset T_{m} M$ centered at $0_{m} \in T_{m} m$ such that $U:=\exp (B)$ is an open neighborhood of $m$, and exp : $B \rightarrow U$ is a diffeomorphism.

Definition 95 Continuing the notation above, let $x:=\left.\exp \right|_{U} ^{-1}: U \rightarrow B \subset T_{m} M \cong$ $\mathbb{R}^{d}$ and for $p=\exp (v) \in U$ with $v=x(p)$ we let

$$
u(p):=/ /_{1}^{\nabla^{E}}\left(\sigma_{v}\right): E_{m} \rightarrow E_{p} .
$$

Hence $(x, U)$ is chart on $M$ centered at $m$ and $u$ is local frame defined on $U \subset M$. [Note that $\sigma_{v}$ and $x$ both depend on $\nabla^{M}$ and $u$ depends on both $\nabla^{M}$ and $\nabla^{E}$ even though this dependence is being suppressed from the notation.]

The next proposition is the key to coordinate free description of " $\stackrel{n}{\sim}$ ”.

Proposition 96 Let $k \in \mathbb{N}_{0}, m \in M, \nabla^{E}$ and $\nabla^{M}$ be any covariant derivatives on $E$ and $T M$ respectively, and $(x, u)$ be the chart/frame as in Definition 95 . Then for any $S \in \Gamma(m)$,

$$
\left.\left(\frac{\nabla^{E}}{d t}\right)^{k} S\left(\sigma_{v}(t)\right)\right|_{t=0}=\left(\partial_{v}^{k}\left[\left(u^{-1} S\right) \circ x^{-1}\right]\right)(x(m)) \text { for all } v \in T_{m} M .
$$


[Note that by construction $x(m)=0_{m} \in T_{m} M$.]

Proof Let $v \in T_{m} M$ be given and then choose $\delta \in(0,1)$ such that $\sigma_{v}(t)$ exists for $t \in[0, \delta]$. Since $\sigma_{t v}(s)=\sigma_{v}(t s),\left.\sigma_{t v}\right|_{[0, \delta]}$ is a reparametrization of $\sigma_{v}$ on $[0, t \delta]$, we may conclude that

$$
u\left(\sigma_{v}(t)\right)=u\left(\sigma_{t v}(1)\right)=/ /_{1}^{\nabla^{E}}\left(\sigma_{t v}\right)=/ /_{t}^{\nabla^{E}}\left(\sigma_{v}\right) \text { for all } 0 \leq t \leq \delta .
$$

Using this observation along with $x^{-1}(t v)=\exp (t v)=\sigma_{v}(t)$, we find

$$
\begin{aligned}
\left(\partial_{v}^{k}\left[\left(u^{-1} S\right) \circ x^{-1}\right]\right)(x(m)) & =\left(\partial_{v}^{k}\left[\left(u^{-1} S\right) \circ x^{-1}\right]\right)(0) \\
& =\left.\left(\frac{d}{d t}\right)^{k}\right|_{t=0}\left(u^{-1} S\right) \circ x^{-1}(t v) \\
& =\left.\left(\frac{d}{d t}\right)^{k}\right|_{t=0}\left[u\left(\sigma_{v}(t)\right)^{-1} S\left(\sigma_{v}(t)\right)\right] \\
& =\left.\left(\frac{d}{d t}\right)^{k}\right|_{t=0}\left[/ /_{t}^{\nabla}\left(\sigma_{v}\right)^{-1} S\left(\sigma_{v}(t)\right)\right] \\
& =\left.\left(\frac{\nabla^{E}}{d t}\right)^{k}\right|_{t=0}\left[S\left(\sigma_{v}(t)\right)\right] .
\end{aligned}
$$

The following is now an immediate corollary of Proposition 96.

Corollary 97 If $n \in \mathbb{N}_{0}, m \in M, \nabla^{E}$ and $\nabla^{M}$ be any covariant derivatives on $E$ and $T M$ respectively, and $S, T \in \Gamma(m)$, then $S \stackrel{n}{\sim} T$ iff

$$
\left.\left(\frac{\nabla^{E}}{d t}\right)^{k}\right|_{t=0}\left[S\left(\sigma_{v}(t)\right)\right]=\left.\left(\frac{\nabla^{E}}{d t}\right)^{k}\right|_{t=0}\left[T\left(\sigma_{v}(t)\right)\right] \text { for } 0 \leq k \leq n \text { and } v \in T_{m} M .
$$

Definition 98 If $S \in \Gamma(m)$ and $n \in \mathbb{N}_{0}$, let $\operatorname{tay}_{m}^{n}(S): T_{m} M \rightarrow E_{m}$ be the function defined for all $v \in T_{m} M$ by

$$
\operatorname{tay}_{m}^{n}(S)(v):=\left.\sum_{k=0}^{n} \frac{1}{k !}\left(\frac{\nabla^{E}}{d t}\right)^{k}\right|_{t=0}\left[S\left(\sigma_{v}(t)\right)\right]
$$

According to Proposition 96, we may rewrite (53) as

$$
\operatorname{tay}_{m}^{n}(S)(v)=\sum_{k=0}^{n} \frac{1}{k !}\left(\partial_{v}^{k}\left[\left(u^{-1} S\right) \circ x^{-1}\right]\right)(0)
$$

which shows that $\operatorname{tay}_{m}^{n}(S) \in \mathcal{P}_{n}\left(T_{m} M, E_{m}\right)$, the linear space of degree $n$-polynomial on $T_{m} M$ with values in $E_{m}$. Let us further note that $D^{k}\left[\left(u^{-1} S\right) \circ x^{-1}\right](0) \in$ 
$\Sigma^{k}\left[T_{m}^{*} M\right]$ is uniquely determined by

$$
\frac{1}{k !} D^{k}\left[\left(u^{-1} S\right) \circ x^{-1}\right](0) v^{\otimes k}=\frac{1}{k !}\left(\partial_{v}^{k}\left[\left(u^{-1} S\right) \circ x^{-1}\right]\right)(0)
$$

where $v \rightarrow \frac{1}{k !}\left(\partial_{v}^{k}\left[\left(u^{-1} S\right) \circ x^{-1}\right]\right)(0)$ is the $k$-homogeneous part of $\operatorname{tay}_{m}^{n}(S)$. Hence we may view $\operatorname{tay}_{m}^{n}(S)$ as taking values in $\Sigma^{k}\left(T_{m}^{*} M\right) \otimes E_{m}$. Finally observe that, by Corollary 97, if $S \stackrel{n}{\sim} T$, then $\operatorname{tay}_{m}^{n}(S)=\operatorname{tay}_{m}^{n}(T)$, i.e. we may view $\operatorname{tay}_{m}^{n}(S)$ to be a function of $j_{m}^{n} S$ rather than of $S$. This simply reflects the fact that if $L$ is any $n$-order differential operator on $\Gamma(E)$ then $(L S)(m)=(L T)(m)$ whenever $S \stackrel{n}{\sim} T$.

Theorem 99 To each $n \in \mathbb{N}_{0}$, the jet bundle $J^{n}(\pi)$ is non-canonically isomorphic to $\oplus_{k=0}^{n}\left[\Sigma^{k}\left(T^{*} M\right) \otimes E\right]$. In more detail, if $\nabla^{E}$ and $\nabla^{M}$ are covariant derivatives on $E$ and $T M$ respectively and $m \in M$, then the associated Taylor map,

$$
\operatorname{tay}_{m}^{n}: J_{m}^{n}(\pi) \rightarrow \oplus_{k=0}^{n}\left[\Sigma^{k}\left(T_{m}^{*} M\right) \otimes E_{m}\right] \cong \mathcal{P}_{n}\left(T_{m} M, E_{m}\right)
$$

is a linear isomorphism of vector spaces.

Proof In the lead up to the statement of the theorem we have already shown tay ${ }_{m}^{n}$ in (54) is a well defined linear map and that its kernel has dimension zero. So to finish the proof we need only show $\operatorname{tay}_{m}^{n}$ is surjective. To this end let $Q \in \mathcal{P}_{n}\left(T_{m} M, E\right)$ which we decompose and let

$$
Q_{k}(v):=\left.\frac{1}{k !}\left(\frac{d}{d t}\right)^{k}\right|_{t=0} Q(t v)
$$

be the $k$-homogeneous part of $Q$. By Taylor's theorem, we know that $Q(v)=$ $\sum_{k=0}^{n} Q_{k}(v)$. Using $(x, u)$ from Definition 95, we then let

$$
S(p):=S_{Q}(p):=u(p) Q(x(p)) \text { for all } p \in U .
$$

Then $S \in \Gamma(m)$ and moreover for each $0 \leq k \leq n$ and $v \in T_{m} M$,

$$
\begin{aligned}
\left.\frac{1}{k !}\left(\frac{\nabla^{E}}{d t}\right)^{k}\right|_{t=0} S\left(\sigma_{v}(t)\right) & =\left.\frac{1}{k !}\left(\frac{d}{d t}\right)^{k}\right|_{t=0}\left[u\left(\sigma_{v}(t)\right)^{-1} S\left(\sigma_{v}(t)\right)\right] \\
& =\left.\frac{1}{k !}\left(\frac{d}{d t}\right)^{k}\right|_{t=0}\left[Q\left(x \circ \sigma_{v}(t)\right)\right] \\
& =\left.\frac{1}{k !}\left(\frac{d}{d t}\right)^{k}\right|_{t=0}[Q(t v)]=Q_{k}(v) .
\end{aligned}
$$

Thus it follows that

$$
\left(\operatorname{tay}_{m}^{n} S_{Q}\right)(v)=\sum_{k=0}^{n} Q_{k}(v)=Q(v)
$$


which shows tay ${ }_{m}^{n}$ is surjective and completes the proof.

In order to write out tay ${ }_{m}^{n}$ more explicitly, let $\nabla$ denote the covariant derivative constructed on any of the bundles $\left[T^{*} M\right]^{\otimes k} \otimes E$ which is constructed from $\nabla^{E}$ and $\nabla^{M}$ in such a way that the product rule holds. Note that $\nabla^{k} S$ is then a section of $\left[T^{*} M\right]^{\otimes k} \otimes E$, so for $v \in T_{m} M$

$$
\left\langle\nabla^{k} S, v^{\otimes k}\right\rangle
$$

is an element of $E_{m}$. This is consistent with earlier notation, where for $E=M \times \mathbb{R}$ the pairing was real valued.

Proposition 100 If $S \in \Gamma(m)$ and $v \in T_{m} M$, then

$$
\left(\operatorname{tay}_{m}^{n} S\right)(v)=\sum_{k=0}^{n} \frac{1}{k !}\left\langle\nabla^{k} S, v^{\otimes k}\right\rangle=\sum_{k=0}^{n} \frac{1}{k !}\left\langle\operatorname{Sym} \nabla^{k} S, v^{\otimes k}\right\rangle
$$

where $\nabla^{k} S=\overbrace{\nabla \cdots \nabla}^{k \text {-times }} S$.

Proof We will show by induction that

$$
\left(\frac{\nabla^{E}}{d t}\right)^{k} S\left(\sigma_{v}(t)\right)=\left\langle\nabla^{k} S, \dot{\sigma}_{v}(t)^{\otimes k}\right\rangle \text { for } k \in \mathbb{N}_{0}
$$

The case $k=0$ is trivial, and the case $k=1$ holds, since

$$
\frac{\nabla^{E}}{d t} S\left(\sigma_{v}(t)\right)=\nabla_{\dot{\sigma}_{v}(t)}^{E} S=\nabla_{\dot{\sigma}_{v}(t)} S=\left\langle\nabla S, \dot{\sigma}_{v}(t)\right\rangle
$$

For the induction step we compute;

$$
\begin{aligned}
\left(\frac{\nabla^{E}}{d t}\right)^{k+1} S\left(\sigma_{v}(t)\right) & =\frac{\nabla^{E}}{d t}\left(\frac{\nabla^{E}}{d t}\right)^{k} S\left(\sigma_{v}(t)\right)=\frac{\nabla^{E}}{d t}\left\langle\nabla^{k} S, \dot{\sigma}_{v}(t)^{\otimes k}\right\rangle \\
& =\left\langle\nabla_{\dot{\sigma}_{v}(t)}^{E} \nabla^{k} S, \dot{\sigma}_{v}(t)^{\otimes k}\right\rangle+\left\langle\nabla^{k} S, \frac{\nabla^{T M^{\otimes k}}}{d t} \dot{\sigma}_{v}(t)^{\otimes k}\right\rangle \\
& =\left\langle\nabla^{k+1} S, \dot{\sigma}_{v}(t)^{\otimes(k+1)}\right\rangle,
\end{aligned}
$$

wherein we have used,

$$
\frac{\nabla^{T M^{\otimes k}}}{d t} \dot{\sigma}_{v}(t)^{\otimes k}=\sum_{j=1}^{k} \dot{\sigma}_{v}(t)^{\otimes(j-1)} \otimes\left[\frac{\nabla^{M}}{d t} \dot{\sigma}_{v}(t)\right] \otimes \dot{\sigma}_{v}(t)^{\otimes(k-j)}=0
$$


as $\sigma_{v}(\cdot)$ is a $\nabla^{M}$-geodesic and the induction step in complete. Evaluating (55) at $t=0$ then shows

$$
\left(\operatorname{tay}_{m}^{n} S\right)(v)=\left.\sum_{k=0}^{n} \frac{1}{k !}\left(\frac{\nabla^{E}}{d t}\right)^{k}\right|_{0} S\left(\sigma_{v}(t)\right)=\sum_{k=0}^{n} \frac{1}{k !}\left\langle\nabla^{k} S, v^{\otimes k}\right\rangle
$$

as desired.

In the case of the trivial bundle $E=M \times \mathbb{R}$, Theorem 99 shows that the jet bundle is just another representation of the "polynomial" regularity structure of the preceeding section. We finish this section, by showing Taylor's theorem in the setting of general vector bundle presented here.

Definition 101 For $n \in \mathbb{N}_{0}, m \in M$, and $S \in \Gamma(m)$, define

$$
\left(\operatorname{Tay}_{m}^{n} S\right)(p):=\left(\operatorname{tay}_{m}^{n} S\right)\left(\exp _{m}^{-1}(p)\right)
$$

for $p \in U=\exp (B)$ as in Definition 95 .

Remark 102 Taking $E=M \times \mathbb{R}$, this is consistent with Definition 65 .

Theorem 103 (Taylor's Theorem) If $S \in \Gamma(E)$ is a smooth section, $m \in M$, and $n \in \mathbb{N}_{0}$, then

$$
S(\exp (v))=/ / 1\left(\sigma_{v}\right)\left[\left(\operatorname{tay}_{m}^{n} S\right)(v)+\rho_{n}(v)\right] \text { for } v \in B
$$

where

$$
\rho_{n}(v):=\frac{1}{n !} \int_{0}^{1} / /{ }_{t}\left(\sigma_{v}\right)^{-1}\left(\frac{\nabla^{E}}{d t}\right)^{(n+1)} S\left(\sigma_{v}(t)\right)(1-t)^{n} d t .
$$

Alternatively we may express (56) as (recall Definition 95 for the definition of $u$ )

$$
S(p)=u(p)\left[\left(\operatorname{Tay}_{m}^{n} S\right)(p)+\rho_{n}\left(\exp _{m}^{-1}(p)\right)\right] \text { for } p \in U
$$

Proof For $v \in B \subset T_{m} M$, let $s(t):=/ /{ }_{t}\left(\sigma_{v}\right)^{-1} S\left(\sigma_{v}(t)\right) \in E_{m}$. Applying Taylor's theorem with integral remainder to $s$ implies

$$
s(1)=\sum_{k=0}^{n} \frac{1}{k !} s^{(k)}(0)+\frac{1}{n !} \int_{0}^{1} s^{(n+1)}(t)(1-t)^{n} d t .
$$

and hence

$$
\begin{aligned}
S(\exp (v)) & =S\left(\sigma_{v}(1)\right)=/ / 1\left(\sigma_{v}\right) s(1) \\
& =/ / 1\left(\sigma_{v}\right)\left[\sum_{k=0}^{n} \frac{1}{k !} s^{(k)}(0)+\frac{1}{n !} \int_{0}^{1} s^{(n+1)}(t)(1-t)^{n} d t\right]
\end{aligned}
$$




$$
\begin{aligned}
& =/ / 1\left(\sigma_{v}\right)\left[\left.\sum_{k=0}^{n} \frac{1}{k !}\left(\frac{\nabla^{E}}{d t}\right)^{k}\right|_{0} S\left(\sigma_{v}(t)\right)+\rho_{n}(v)\right] \\
& =/ / 1\left(\sigma_{v}\right)\left[\left(\operatorname{tay}_{m}^{n} S\right)(v)+\rho_{n}(v)\right]
\end{aligned}
$$

where

$$
\rho_{n}(v)=\frac{1}{n !} \int_{0}^{1} s^{(n+1)}(t)(1-t)^{n} d t .
$$

Combining (57) and (58) with the identity,

$$
s^{(n+1)}(t)=\left(\frac{d}{d t}\right)^{(n+1)}\left[/ / t\left(\sigma_{v}\right)^{-1} S\left(\sigma_{v}(t)\right)\right]=/ / t\left(\sigma_{v}\right)^{-1}\left(\frac{\nabla^{E}}{d t}\right)^{(n+1)} S\left(\sigma_{v}(t)\right),
$$

completes the proof.

Acknowledgements Open access funding provided by Max Planck Society. The authors wish to thank the referees for useful comments and suggestions, especially for pointing out the relation with jet bundle. The first author was supported in part by RTG 1845 and EPSRC grant EP/I03372X/1. The second author has received funding by the DAAD P.R.I.M.E. program. He would like to thank Giuseppe Cannizzaro and Konstantin Matetski for discussion on the Schauder estimates.

Open Access This article is distributed under the terms of the Creative Commons Attribution 4.0 International License (http://creativecommons.org/licenses/by/4.0/), which permits unrestricted use, distribution, and reproduction in any medium, provided you give appropriate credit to the original author(s) and the source, provide a link to the Creative Commons license, and indicate if changes were made.

\section{References}

1. Berline, N., Getzler, E., Michele, V.: Heat Kernels and Dirac Operators. Springer, Berlin (1992)

2. Bailleul, I., Frédéric, B.: Heat semigroup and singular PDEs. J. Funct. Anal. 270(9), 3344-3452 (2016)

3. Bailleul, I., Frédéric, B.: Higher order paracontrolled calculus (2016). arXiv preprint arXiv:1609.06966

4. Cannizzaro, G., Konstantin, M.: Space-time discrete KPZ equation (2016). arXiv preprint arXiv:1611.09719

5. Driver, B., Semko, J.: Controlled rough paths on manifolds I. Rev. Mat. Iberoam. 33(3), 885-950 (2017)

6. Friedlander, F.G.: The Wave Equation on a Curved Space-Time, vol. 2. Cambridge University Press, Cambridge (1975)

7. Gubinelli, M., Peter, I., Nicolas, P.: Paracontrolled Distributions and Singular PDEs. Forum of Mathematics, Pi, vol. 3. Cambridge University Press, Cambridge (2015)

8. Janson, S.: Gaussian Hilbert Spaces. Cambridge Tracts in Mathematics, vol. 129. Cambridge University Press, Cambridge (1997)

9. Hairer, M.: A theory of regularity structures. Invent. Math. 198(2), 269-504 (2014)

10. Hairer, M.: Introduction to regularity structures. Braz. J. Probab. Stat. 29(2), 175-210 (2015)

11. Hairer, M., Konstantin, M.: Discretisations of rough stochastic PDEs (2015). arXiv preprint arXiv: 1511.06937

12. Hörmander, L.: Linear Partial Differential Operators, vol. 116. Springer, Berlin (2013)

13. Lee, J.M.: Introduction to Smooth Manifolds. Springer, New York (2003)

14. Lee, J.M.: Riemannian Manifolds: An Introduction to Curvature, vol. 176. Springer, Berlin (2006)

15. Rosenberg, S.: The Laplacian on a Riemannian Manifold: An Introduction to Analysis on Manifolds, vol. 31. Cambridge University Press, Cambridge (1997)

16. Saunders, D.J.: The Geometry of Jet Bundles, vol. 142. Cambridge University Press, Cambridge (1989)

17. Waldmann, S.: Geometric wave equations (2012). arXiv preprint arXiv:1208.4706 